TRANSACTIONS OF THE

AMERICAN MATHEMATICAL SOCIETY

Volume 352, Number 3, Pages 969-1003

S 0002-9947(99)02554-4

Article electronically published on October 15, 1999

\title{
THE CALCULUS OF PARTITION SEQUENCES, CHANGING COFINALITIES, AND A QUESTION OF WOODIN
}

\author{
ARTHUR W. APTER, JAMES M. HENLE, AND STEPHEN C. JACKSON
}

\begin{abstract}
We study in this paper polarized infinite exponent partition relations. We apply our results to constructing a model for the theory " $\mathrm{ZF}+\mathrm{DC}+\omega_{1}$ is the only regular, uncountable cardinal $\leq \omega_{\omega_{1}+1}$." This gives a partial answer to a question of Woodin.
\end{abstract}

In 1994, J. Steel proved what had long been suspected: that assuming AD, the regular cardinals of $L(\mathbb{R})$ below $\Theta$ are all measurable, where $\Theta$ is the least ordinal onto which the real numbers $\mathbb{R}$ cannot be mapped [St3]. This remarkable theorem motivated the work of $[\mathrm{A}]$, which showed essentially that the cofinality of any regular cardinal below $\Theta$ can be changed to $\omega$ without perturbing cardinal structure. Specifically, the following theorem was proved.

Theorem 0.1. Assume $\mathbf{V}^{\prime} \vDash \mathrm{AD}$, and let $\mathbf{V}=L(\mathbb{R}) \mathbf{V}^{\prime}$. Then for any subset $A$ of the regular, uncountable cardinals below $\Theta^{\mathbf{V}}$, there is a partial ordering $\mathcal{P} \in \mathbf{V}$ and a symmetric inner model $\mathbf{N}$ of $\mathrm{ZF}$ such that

(1) $\mathbf{V} \subseteq \mathbf{N} \subseteq \mathbf{V}^{\mathcal{P}}$,

(2) $\mathbf{N}$ and $\mathbf{V}$ contain the same cardinals,

(3) $\Theta^{\mathbf{N}}=\Theta^{\mathbf{V}}$,

(4) for all $\kappa, \operatorname{cof}^{\mathbf{N}}(\kappa)=\omega$ if $\operatorname{cof}^{\mathbf{V}}(\kappa) \in A$ and $\operatorname{cof}^{\mathbf{N}}(\kappa)=\operatorname{cof}^{\mathbf{V}}(\kappa)$ otherwise, and

(5) the measurable cardinals not in $A$ remain measurable in $\mathbf{N}$.

What was left unanswered is whether similar facts can be proven if the cofinality of $\kappa<\Theta$ is to be made uncountable.

Consideration of this question was the genesis of this paper. The principal tool, infinite-exponent partition sequences, proved as interesting as its application.

We begin, in the first three sections, with background material on infiniteexponent partition properties of cardinals, their abundance in models of AD, and their use in "Magidor-like" forcing to change cofinalities.

The second three sections deal with infinite-exponent partition properties of cardinal sequences. With appropriate notation, the basic theory of partition sequences and the associated Magidor-like forcing exactly parallels the cardinal theory.

In "Finite Support," we apply the theory to show the extent to which possibly uncountable cofinal sequences can be added if we sacrifice DC. In "Countable Support," we preserve DC.

Received by the editors September 30, 1997.

1991 Mathematics Subject Classification. Primary 03E15, 03E35, 03E60.

The first author's research was partially supported by PSC-CUNY grant 665337.

(C)1999 American Mathematical Society 
In general, uncountable cofinalities present difficulties. In [G], M. Gitik established the consistency relative to an almost huge cardinal of the theory " $\mathrm{ZF}+\omega_{1}$ is the only regular, uncountable cardinal." In his model, all cardinals above $\omega_{1}$ are given cofinality $\omega$ and consequently $\mathrm{DC}$ fails (in fact $\mathrm{AC}_{\omega}$ fails). $\mathrm{DC}$ is most desirable in analysis. A. Kechris proved that DC holds in $L(\mathbb{R})$ assuming $\mathrm{AD}[\mathrm{Ke} 2]$. W. $\mathrm{H}$. Woodin has asked if the theory " $\mathrm{ZF}+\mathrm{DC}+\omega_{1}$ is the only regular, uncountable cardinal" is consistent.

"Including Ultrapowers" and "Including Ultraproducts" discuss how ultrapowers and ultraproducts of cardinals in the most powerful partition sequences can be included in new but less powerful partition sequences.

The final section applies our results to obtaining a partial answer to Woodin's question. In particular, relative to $\operatorname{Con}(\mathrm{AD})$, we construct a model for the theory $" \mathrm{ZF}+\mathrm{DC}+\omega_{1}$ is the only regular, uncountable cardinal $\leq \omega_{\omega_{1}+1} . "$

Some remarks on notation: When forcing, we will use $\mathbf{V}[G]$ and $\mathbf{V}^{\mathcal{P}}$ interchangeably to indicate the generic extension obtained when $\mathcal{P}$ is our partial ordering, $G$, the generic set. If $p \in \mathcal{P}$ is a condition and $\phi$ is a formula in the forcing language with respect to $\mathcal{P}$, then $p \| \phi$ will mean that $p$ decides $\phi$. We adopt the NESTS convention for the forcing partial order; that is, if $p, q \in \mathcal{P}$ and $q$ extends $p$, we will write $q \Vdash p$.

We will refer to and use certain weak versions of the Axiom of Choice throughout the course of this paper. $\mathrm{AC}_{\omega}$, the Principle of Countable Choice, says that if $\left\langle X_{n}\right.$ : $n\langle\omega\rangle$ is an $\omega$-sequence of non-empty sets, then $\prod_{n<\omega} X_{n}$ is non-empty. DC, the Principle of Dependent Choices, says that if $R \subseteq X \times X$ satisfies $\forall x \exists y[\langle x, y\rangle \in R]$, $X \neq \emptyset$, then there is a sequence $\left\{x_{n}\right\}_{n<\omega}$ of elements of $X$ with $\left\langle x_{n}, x_{n+1}\right\rangle \in R$, for each $n<\omega$.

If $s$ is a set of ordinals, we use $\bar{s}$ to indicate the order-type of $s$. If $x$ and $y$ are sequences, $x \frown y$ denotes their concatenation.

General references for results on AD include [Mo2] and [St1].

\section{PARTITION CARDinAls}

We assume the reader is familiar with infinite-exponent partition cardinals, but we include the definitions in this section for convenience. [H1], [H2], [H3], [K11], [Kl2], and [Kl3] can be consulted for matters left unexplained here.

For cardinals $\kappa$ and $\gamma,[\kappa]^{\gamma}$ is the set of all subsets of $\kappa$ of order-type $\gamma$. We warn readers that we will identify elements of $[\kappa]^{\gamma}$ with their increasing enumerations. In practice, this will cause no confusion; the meaning will follow from the context. We define $[p]^{\gamma}$ as $\{q \subseteq p: \bar{q}=\gamma\}$, for $p \in[\kappa]^{\gamma}$, for example, and it is clear we are treating $p$ as a set. Similarly, we define $\omega p$ as

$$
\left\{\bigcup_{n<\omega} p(\alpha+n): \alpha<\gamma\right\}
$$

i.e., the set of successive $\omega$-sups of elements of $p$, and it is clear we are treating $p$ as a sequence.

Definition 1.1. For ordinals $\kappa, \gamma, \delta$,

$$
\kappa \rightarrow(\kappa)_{\delta}^{\gamma}
$$

means that for all partitions $f:[\kappa]^{\gamma} \rightarrow \delta$, there is a set $X \in[\kappa]^{\kappa}$ such that $f$ is constant on $[X]^{\gamma}$. $X$ is called homogeneous for $f$. If $\delta=2$, the subscript is omitted, and we write simply $\kappa \rightarrow(\kappa)^{\gamma}$. 
For ordinals $\kappa, \gamma, \delta$, the relation $\kappa \rightarrow(\kappa)_{\delta}^{<\gamma}$ means that for all partitions $f$ : $\bigcup_{\beta<\gamma}[\kappa]^{\beta} \rightarrow \delta$, there is a set $X \in[\kappa]^{\kappa}$ such that $f$ is constant on $[X]^{\beta}$, for all $\beta<\gamma$.

A few elementary results:

Fact 1.2. If $\beta<\gamma$, then $\kappa \rightarrow(\kappa)_{\delta}^{\gamma}$ implies $\kappa \rightarrow(\kappa)_{\delta}^{\beta}$.

Fact 1.3. If $\kappa \rightarrow(\kappa)^{<\gamma}$, then $\kappa \rightarrow(\kappa)_{2^{\delta}}^{<\gamma}$, for all $\delta<\kappa$. (See [H1].)

Fact 1.4. If $\kappa \rightarrow(\kappa)^{\omega+\omega}$, then $\kappa$ is measurable. (See [Kl3].)

Fact 1.5. If $\gamma$ is a cardinal, then $\kappa \rightarrow(\kappa)^{\gamma}$ implies $\kappa \rightarrow(\kappa)^{<\gamma}$. (See [H1].)

Fact 1.6. (DC) If $\kappa \rightarrow(\kappa)^{\kappa}$, then $\kappa \rightarrow(\kappa)_{\omega}^{\kappa}$. (See [H4].)

Fact 1.7. If $\kappa \rightarrow(\kappa)^{\gamma+\gamma}$, then $\kappa \rightarrow(\kappa)_{\alpha}^{\gamma}$ for all $\alpha<\kappa$. (See [Kl3].)

Definition 1.8. A cardinal satisfying $\kappa \rightarrow(\kappa)^{\kappa}$ is called a strong partition cardinal, or simply, strong.

This definition reflects current usage of the term, though originally ([KeKlMW]) strong partition cardinals were required to satisfy $\kappa \rightarrow(\kappa)_{\gamma}^{\kappa}$, for all $\gamma<\kappa$.

For proving instances of partition properties under AD, we will need an alternative formulation of this property. For the rest of this section, we assume AD+DC.

We say a function $F: \lambda \rightarrow O N$ has uniform cofinality $\omega$ if there is a function $F^{\prime}: \omega \times \lambda \rightarrow O N$ which is strictly increasing in the first argument and is such that $\forall \beta<\lambda F(\beta)=\sup ^{\prime} F^{\prime}(n, \beta)$. We say $F: \lambda \rightarrow O N$ is of the correct type if $F$ is increasing, of uniform cofinality $\omega$, and everywhere discontinuous; that is, $\forall \beta<\lambda F(\beta)>\sup _{\beta^{\prime}<\beta} F\left(\beta^{\prime}\right)$.

" $\kappa$ strong" is equivalent to the statement: for every partition $\mathcal{P}:[\kappa]^{\kappa} \rightarrow\{0,1\}$, there is an $i \in\{0,1\}$ and a c.u.b. $C \subseteq \kappa$ such that $\mathcal{P}(F)=i$ for all $F: \kappa \rightarrow C$ of the correct type. The equivalence of these two definitions is a straightforward partition argument left to the reader.

In Definition 1.9 below, we isolate the "minimal hypothesis" on a cardinal $\kappa$ from which one can carry out Martin's proof of the strong partition relation.

Definition 1.9. A regular cardinal $\kappa$ is said to be reasonable if there is a nonselfdual pointclass $\Gamma$ closed under $\exists^{\omega^{\omega}}$ and a map $\phi$ with domain $\omega^{\omega}$ satisfying:

1. $\forall x \phi(x) \subseteq \kappa \times \kappa$.

2. For every function $F: \kappa \rightarrow \kappa \exists x \phi(x)=F$.

3. $\forall \beta, \gamma<\kappa R_{\beta, \gamma} \in \boldsymbol{\Delta} \doteq \boldsymbol{\Gamma} \cap \check{\boldsymbol{\Gamma}}$, where $x \in R_{\beta, \gamma} \leftrightarrow \phi(x)(\beta, \gamma) \wedge \forall \gamma^{\prime}<$ $\kappa\left(\phi(x)\left(\beta, \gamma^{\prime}\right) \rightarrow \gamma^{\prime}=\gamma\right)$.

4. Suppose $\beta<\kappa, A \in \exists^{\omega^{\omega}} \Delta$, and $A \subseteq R_{\beta} \doteq\left\{x: \exists \gamma<\kappa R_{\beta, \gamma}(x)\right\}$. Then $\exists \gamma_{0}<\kappa \forall x \in A \exists \gamma<\gamma_{0} R_{\beta, \gamma}(x)$.

For $\kappa$ reasonable, we will use the notation $R_{\beta}, R_{\beta, \gamma}$ as above (of course, this depends on the coding function $\phi)$. Also, if $x \in R_{\beta}$, we let $\phi(x)(\beta)$ denote the unique $\gamma$ such that $\phi(x)(\beta, \gamma)$.

We present a sketch of Martin's proof, assuming $\mathrm{AD}+\mathrm{DC}$, that such cardinals have the strong partition property. We will show later that $\boldsymbol{\Gamma}$ is necessarily closed under finite (in fact countable) unions and intersections, and $\boldsymbol{\Delta}$ is closed under $<\kappa$ unions and intersections; we borrow these facts for the following proof and 
Proposition 5.3. One more useful general fact which we use below, and later, is that if $\boldsymbol{\Gamma}$ is a non-selfdual pointclass with $\boldsymbol{\Gamma}=\exists^{\omega^{\omega}} \boldsymbol{\Delta}$ (where $\boldsymbol{\Delta}=\boldsymbol{\Gamma} \cap \check{\boldsymbol{\Gamma}}$ ), then $\boldsymbol{\Gamma}$ is closed under finite unions and intersections. To see this, note that if $\boldsymbol{\Gamma}=\exists^{\omega^{\omega}} \boldsymbol{\Lambda}$ where $\boldsymbol{\Lambda}$ is non-selfdual and closed under $\forall^{\omega^{\omega}}$, then it is easy to see directly that $\boldsymbol{\Gamma}$ is closed under countable unions and intersections. The only remaining case (cf. [St1]) is when $\boldsymbol{\Gamma}$ is the base of a type I hierarchy. That is, $\boldsymbol{\Gamma}=\bigcup_{\omega} \boldsymbol{\Lambda}$ for some selfdual $\boldsymbol{\Lambda}$ closed under quantifiers (and where $\operatorname{cof}(o(\boldsymbol{\Lambda}))=\omega$ ). In this case, easily $\boldsymbol{\Gamma}$ is closed under finite intersections.

Proposition 1.10 (Martin). Every reasonable $\kappa$ has the strong partition property.

Proof. Fix a partition $\mathcal{P}:[\kappa]^{\kappa} \rightarrow\{0,1\}$. Play the integer game where I plays out $x \in \omega^{\omega}$, II plays out $y \in \omega^{\omega}$. If there is a least ordinal $\beta<\kappa$ such that $x \notin R_{\beta}$ or $y \notin R_{\beta}$, then II wins provided $x \notin R_{\beta}$. Otherwise, let $f_{x}, f_{y}: \kappa \rightarrow \kappa$ be the functions they determine. Define $f_{x, y}: \kappa \rightarrow \kappa$ by $f_{x, y}(\beta)=\sup _{\beta^{\prime}<\omega \cdot(\beta+1)} \max \left(f_{x}\left(\beta^{\prime}\right), f_{y}\left(\beta^{\prime}\right)\right)$. II then wins iff $\mathcal{P}\left(f_{x, y}\right)=1$.

Assume without loss of generality that II has a winning strategy $\tau$. Define $x \in S_{\beta, \gamma} \leftrightarrow \forall \beta^{\prime} \leq \beta \exists \gamma^{\prime} \leq \gamma x \in R_{\beta^{\prime}, \gamma^{\prime}}$. Thus, $S_{\beta, \gamma} \in \Delta$. An easy computation shows that $\forall \beta, \gamma<\kappa \tau\left[S_{\beta, \gamma}\right] \in \exists^{\omega^{\omega}} \boldsymbol{\Delta}$ (take cases as to whether $\exists^{\omega^{\omega}} \boldsymbol{\Delta}=\boldsymbol{\Gamma}$, and use the closure of $\boldsymbol{\Gamma}$ under $\wedge$ in this case). Now, $\forall y \in \tau\left[S_{\beta, \gamma}\right] y \in R_{\beta}$. Thus, $\theta(\beta, \gamma) \doteq \sup \left\{\phi(x)(\beta): x \in \tau\left[S_{\beta, \gamma}\right]\right\}<\kappa$. Let $C \subseteq \kappa$ be the set of points closed under $\theta$, and $C^{\prime} \subseteq C$ the set of limit points of $\mathrm{C}$.

Suppose $F: \kappa \rightarrow C^{\prime}$ is of the correct type. We show that $\mathcal{P}(F)=1$. Let $x$ be such that $\phi(x)$ determines a function $f_{x}: \kappa \rightarrow C$ such that $F(\beta)=\sup _{\beta^{\prime}<\omega \cdot(\beta+1)} f_{x}\left(\beta^{\prime}\right)$. We may assume $f_{x}(\beta) \geq \beta$ for all $\beta$. Let $y=\tau(x)$. Easily $\phi(y)$ determines a function $f_{y}: \kappa \rightarrow \kappa$ and $f_{y}\left(\beta^{\prime}\right) \leq f_{x}(\beta+1)$ for all $\beta$. Thus, $F=f_{x, y}$, so $\mathcal{P}(F)=1$.

We now introduce a slight strengthening of the notion of reasonableness.

Definition 1.11. A regular cardinal $\kappa$ is very reasonable if there are $\boldsymbol{\Gamma}, \phi$ witnessing $\kappa$ is reasonable, and for all $\beta<\kappa$ there is an $\exists{ }^{\omega} \boldsymbol{\Delta}$ relation $B \subseteq \omega^{\omega} \times \omega^{\omega}$ such that for $x, y \in R_{\beta}, \phi(x)(\beta)<\phi(y)(\beta) \leftrightarrow B(x, y)$.

We note again that in all cases where the strong partition relation on $\kappa$ has been shown to hold, $\kappa$ has been shown to be very reasonable.

\section{AD AND PARTITION CARDINALS}

Our focus in this paper is on models of $\mathrm{AD}$ plus $\mathbf{V}=L(\mathbb{R})$. As noted, this implies DC as well. Under these assumptions, there are many partition cardinals. Conceivably, all regular cardinals below $\Theta$ possess some sort of infinite-exponent partition property.

Martin showed that the first projective ordinal, $\delta_{1}^{1}=\omega_{1}$, has the strong partition property. Martin and Solovay also computed $\delta_{3}^{1}=\omega_{\omega+1}$ and showed that the only regular cardinal strictly between $\boldsymbol{\delta}_{1}^{1}$ and $\boldsymbol{\delta}_{3}^{1}$ is $\omega_{2}$, which is the ultrapower of $\boldsymbol{\delta}_{1}^{1}$ by the unique normal measure on $\boldsymbol{\delta}_{1}^{1}$ (given by the c.u.b. filter).

Jackson ([J1], [J3]) later proved the strong partition relation on $\boldsymbol{\delta}_{3}^{1}$, computed $\boldsymbol{\delta}_{5}^{1}=\omega_{\omega^{\omega \omega}}+1$, and showed that the only regular cardinals strictly between $\boldsymbol{\delta}_{3}^{1}$ and $\boldsymbol{\delta}_{5}^{1}$ are the ultrapowers of $\boldsymbol{\delta}_{3}^{1}$ by the three normal measures on $\boldsymbol{\delta}_{3}^{1}$ (generated by the c.u.b. filter and points of cofinality $\omega, \omega_{1}$, or $\omega_{2}$ respectively). These three 
regular cardinals turn out to be $\boldsymbol{\delta}_{4}^{1}=\omega_{\omega+2}, \omega_{\omega \cdot 2+1}$, and $\omega_{\omega^{\omega}+1}$. (In [JKh] some additional results along these lines are obtained. For example, the cofinality of all the successor cardinals between $\boldsymbol{\delta}_{3}^{1}$ and $\boldsymbol{\delta}_{5}^{1}$ is determined.)

In [J2] it is shown how to generalize the main ideas of [J1] (the theory of "descriptions") to the general projective level. One thus gets the strong partition property for all the $\boldsymbol{\delta}_{2 n+1}^{1}$, and likewise every regular cardinal strictly between $\boldsymbol{\delta}_{2 n+1}^{1}$ and $\boldsymbol{\delta}_{2 n+3}^{1}$ is an ultrapower of $\boldsymbol{\delta}_{2 n+1}^{1}$ by one of the $\left(2^{n+1}-1\right.$ many) normal measures on $\boldsymbol{\delta}_{2 n+1}^{1}$. Although the full details of the general projective case have not yet appeared, they are basically the proofs of [J1] augmented with the general theory of descriptions of [J2].

In fact, the projective ordinal analysis extends with essentially no modifications to the transfinite $\boldsymbol{\delta}_{\alpha}^{1}$, for $\alpha<\omega_{1}$. Thus, all of the "odd" $\boldsymbol{\delta}_{\alpha}^{1}$ (as usual, limit ordinals are regarded as even) have the strong partition property, and every regular $\kappa<\sup \left\{\boldsymbol{\delta}_{\alpha}^{1}: \alpha<\omega_{1}\right\}=\omega_{\omega_{1}}$ is the ultrapower of an odd $\boldsymbol{\delta}_{\alpha}^{1}$ by a normal measure on it.

Past $\omega_{\omega_{1}}$, the arguments of [J1], [J2] no longer suffice for the $\boldsymbol{\delta}_{\alpha}^{1}$ analysis. Although somewhat tentative (and not written up), unpublished work appears to show that this generalized projective analysis (the "very fine structure of $L(\mathbb{R})$ ") can be extended to the first inaccessible cardinal in $L(\mathbb{R})$. Up to this point, it appears that every regular Suslin cardinal has the strong partition property, and that every regular cardinal is an ultraproduct of regular Suslin cardinals by a normal measure. Although these results must be regarded as tentative, they would nevertheless, combined with Corollary 5.12, allow us to extend the results of the final section up to the first inacessible. Some of the problems arising in attempting to extend the $L(\mathbb{R})$ analysis further are discussed in [J4].

We conjecture (assuming $\mathrm{AD}+\mathbf{V}=L(\mathbb{R})$ ), that every regular Suslin cardinal $\kappa<\Theta$ has the strong partition property, and every regular $\lambda<\Theta$ is an ultraproduct of regular Suslin cardinals by a normal measure. Granting this, the results of the final section can be extended to $\Theta$, although Theorem 5.10 falls short of showing that every (increasing, discontinuous) sequence of very reasonable cardinals (see section 5) has the strong polarized partition property.

\section{MAGIDOR-LIKE FORCING}

Assuming that $\kappa \rightarrow(\kappa)^{<\gamma}$ and that $\gamma$ is a regular, uncountable cardinal, "Magidor-like" forcing changes the cofinality of $\kappa$ to $\gamma$ without adding any bounded subsets to $\kappa$ (thereby preserving the fact that $\kappa$ is a cardinal). No use is made here of $\mathrm{AD}+\mathrm{DC}$. The conditions are defined as follows.

Definition 3.1. We define the set $\mathcal{P}_{\gamma, \kappa}$ by

$$
\mathcal{P}_{\gamma, \kappa}=\left\{\langle s, x\rangle: s \in[\kappa]^{<\gamma}, x \in[\kappa]^{\kappa}, \bigcup s<\bigcap x\right\} .
$$

The partial ordering for $\mathcal{P}_{\gamma, \kappa}$ is: $\left\langle s^{\prime}, x^{\prime}\right\rangle \Vdash\langle s, x\rangle$ iff $s \subseteq s^{\prime},\left\langle x^{\prime}\right\rangle \subseteq\langle x\rangle$, and $s^{\prime} \backslash s={ }_{\omega} t$ for some $t \in[x]^{<\gamma}$. We use $\langle x\rangle$ to denote $\left\{{ }_{\omega} q: q \in[x]^{\kappa}\right\}$. For $p \in \mathcal{P}_{\gamma, \kappa}$, we denote the coordinates of $p$ by $p_{0}$ and $p_{1}$, i.e., $p=\left\langle p_{0}, p_{1}\right\rangle$.

The sets $\langle x\rangle$ generate a filter on $[\kappa]^{\kappa}$. With the strong partition property, this is a $\kappa$-complete ultrafilter $([\mathrm{H} 3])$. Note that any two conditions $p, p^{\prime}$ are compatible if $p_{0}=p_{0}^{\prime}$. 
Given $\kappa \rightarrow(\kappa)^{<\gamma}$, and $\gamma$, a regular, uncountable cardinal, Propositions 1.1, 1.3, and 1.5 of [H2] show the following:

Fact 3.2. If $\phi$ is a formula in the forcing language with respect to $\mathcal{P}_{\gamma, \kappa}$ and $p \in$ $\mathcal{P}_{\gamma, \kappa}$, then there is an $x \in[\kappa]^{\kappa}$ such that $\left\langle p_{0}, x\right\rangle \| \phi$.

Fact 3.3. $\mathbf{V}^{\mathcal{P}_{\gamma, \kappa}}$ and $\mathbf{V}$ have the same bounded subsets of $\kappa$.

Fact 3.4. The cofinalities of cardinals in $\mathbf{V}^{\mathcal{P}_{\gamma, \kappa}}$ are the same as in $\mathbf{V}$, except that a cardinal having cofinality $\kappa$ in $\mathbf{V}$ has cofinality $\gamma$ in $\mathbf{V}^{\mathcal{P}_{\gamma, \kappa}}$.

We will sometimes be interested in changing the cofinality of $\kappa$ to $\omega$ instead of to an uncountable regular cardinal. $\kappa \rightarrow(\kappa)^{<\omega \cdot \omega}$ is sufficient to show that $\mathcal{P}_{\omega \cdot \omega, \kappa}$ changes the cofinality of $\kappa$ to $\omega$ and also satisfies Propositions 1.1, 1.3, and 1.5 of [H2].

We can improve on [H2]. First we add some notation, in light of Fact 3.2. For $s \in[\kappa]^{<\gamma}$, we will write $s \| \sim \phi$ iff for some $x \in[\kappa]^{\kappa},\langle s, x\rangle \Vdash \phi$. Read this as " $s$ insinuates $\phi$ ". Note that by compatibility, it is impossible for $s$ to insinuate contradictory statements.

Proposition 3.5. If $\kappa$ satisfies $\kappa \rightarrow(\kappa)^{\kappa}$, then forcing with $\mathcal{P}_{\gamma, \kappa}, \gamma<\kappa$, preserves all cardinals.

Proof. By Fact 1.2 and Fact $1.5, \kappa \rightarrow(\kappa)^{<\gamma}$ and hence, by Fact 3.3, no bounded subsets of $\kappa$ are added. This guarantees that cardinals $\kappa$ and below are preserved.

Suppose that $\zeta$ is a cardinal greater than $\kappa$, and $p \Vdash " \dot{f}: \delta \rightarrow \zeta$, onto." For any $s \in\left[p_{1}\right]^{<\gamma}$, let $n_{s}=\left\{\beta<\zeta: \exists \alpha<\delta\left(p_{0} \frown{ }_{\omega} s\right) \mid \sim \dot{f}(\alpha)=\beta\right\}$. We must have $\left\|n_{s}\right\| \leq \delta<\zeta$, since $n_{s} \in \mathbf{V}$ and $\zeta$ is a cardinal in $\mathbf{V}$.

For $t \in\left[p_{1}\right]^{\kappa}$, let $n_{t}=\bigcup\left\{n_{t \uparrow(\beta \backslash \alpha)}: \alpha<\beta, \overline{\beta \backslash \alpha}<\gamma\right\}$ i.e., the union of all $n_{s}$, where $s$ is an interval of $t$ of order-type less than $\gamma$. There are exactly $\kappa$ of these intervals, $t \uparrow(\beta \backslash \alpha)$, and each comes with a natural mapping from a subset of $\delta$ into $\zeta$. Consequently $\left\|n_{t}\right\|<\zeta$, otherwise we would have a mapping in $\mathbf{V}$ from $\delta \times \kappa$ onto $\zeta$.

Let $h:\left[p_{1}\right]^{\kappa} \rightarrow \zeta$ be defined by:

$$
h(t)=\bigcap\left(\zeta \backslash n_{t}\right) .
$$

Define $g:\left[p_{1}\right]^{\kappa} \rightarrow 2$ by

$$
g(t)=0 \text { iff } q \in[t]^{\kappa} \text { implies } h(t) \leq h(q) .
$$

Let $q \in\left[p_{1}\right]^{\kappa}$ be homogeneous for $g \cdot g^{\prime \prime}[q]^{\kappa}=\{0\}$, since if we pick $t \in[q]^{\kappa}$ with $h(t)$ least possible, we will have $g(t)=0$. Let $\beta=h(q)$. Choose $s \in[q]^{<\gamma}$ with $\beta \in n_{s}$. Let $r=q \backslash \bigcup s$. Then $h(q) \leq h(s \cup r) \leq h(r)$ by homogeneity for $g$. But by definition, $h(r) \leq h(q)$, since any interval of $r$ is an interval of $q$. Thus, all these: $h(r), h(q), h(s \cup r)$, are equal and equal to $\beta$. This is impossible, however, as $\beta \in n_{s} \subseteq n_{s \cup r}$.

\section{Partition Sequences}

More powerful than partition cardinals are sequences of such cardinals satisfying "polarized partition properties." The results presented here make no use of AD+DC except as noted in the counterexample below. 
Definition 4.1. Suppose $k$ is a sequence of cardinals and $\delta$ is an ordinal. We write:

$$
(k) \rightarrow(k)_{\delta}^{k}
$$

if for all $f: \prod_{\kappa \in k}[\kappa]^{\kappa} \rightarrow \delta$, there is a sequence $C \in \prod_{\kappa \in k}[\kappa]^{\kappa}$ such that $f$ is constant on $\prod_{\kappa \in k}[C(\alpha)]^{\kappa}$. This is a streamlined version of the original notation, due to Erdös and Rado ([ER]):

$$
\left(\begin{array}{c}
k(0) \\
k(1) \\
\vdots
\end{array}\right) \rightarrow\left(\begin{array}{c}
k(0) \\
k(1) \\
\vdots
\end{array}\right)_{\delta}^{k(0), k(1), \ldots} .
$$

$C$ is called homogeneous for $f$. If $\delta=2$, the subscript is omitted, and we write simply $(k) \rightarrow(k)^{k}$.

We may on occasion consider properties in which the exponents are taken from an arbitrary sequence $d$ of ordinals. We write this: $(k) \rightarrow(k)^{d}$. If each element in the sequence $d$ is the same, $\delta$, we will write: $(k) \rightarrow(k)^{\delta}$.

We will also be working with "Ramsey-like" infinite-exponent polarized partition relations. Specifically, we will write: $(k) \rightarrow(k)^{<\delta}$ to mean that if $f$ : $\bigcup_{\beta<\delta}\left(\prod_{\kappa \in k}[\kappa]^{\beta}\right) \rightarrow 2$ is a partition, then there is a sequence $C \in \prod_{\kappa \in k}[\kappa]^{\kappa}$ such that for each $\beta<\delta, f$ is constant on $\prod_{\kappa \in k}[C(\alpha)]^{\beta}$. Note that we only consider partitions on products where each factor has the same length. A more extravagant definition of exponent " $<\delta$ " can fail even for $\delta=\omega$. (Assume AD and let $k$ be a sequence of cardinals below $\Theta$. Suppose we allowed partitions of $[k]^{\sigma}$ for all $\sigma \in[\omega]^{\omega}$. Since $k(0)<\Theta$, there is a surjection $s:[\omega]^{\omega} \rightarrow k(0)$. Then the partition $f$ on $\bigcup_{\sigma \in[\omega] \omega}[k]^{\sigma}$ defined by $f(p)=0$ iff $p(0)<s(\sigma)$ is a counterexample.)

For brevity and to emphasize the connection between sequences and cardinals, we define further:

Definition 4.2. For $k$ a sequence of cardinals,

$$
[k]^{k}=\prod_{\kappa \in k}[\kappa]^{\kappa} .
$$

Similarly, if $x \in[k]^{k}$, then $[x]^{k}=\prod_{\kappa \in k}[x(\kappa)]^{\kappa}$. If $\gamma$ is a cardinal, then $[k]^{\gamma}=$ $\prod_{\kappa \in k}[\kappa]^{\gamma}$, and $[k]^{<\gamma}=\bigcup_{\beta<\gamma}\left(\prod_{\kappa \in k}[\kappa]^{\beta}\right)$, with $[x]^{\gamma}$ and $[x]^{<\gamma}$ defined similarly. If $x, y \in[k]^{\gamma}$, then $x<y$ means that $\bigcup x(\kappa)<\bigcap y(\kappa)$ for each $\kappa \in k$. If $\alpha$ is an ordinal, then $\alpha<x, x<\alpha$ are defined similarly. In the same manner, define all operations, $x \backslash y$, for example, component-wise. Finally, let $x^{\frown} y$ denote the sequence: $\prod_{\kappa \in k}[x(\kappa) \frown y(\kappa)]$.

We list a few results. The proofs are either elementary or exactly analogous to corresponding facts for partition cardinals.

Fact 4.3. If $\beta<\gamma$, then $(k) \rightarrow(k)_{\delta}^{\gamma}$ implies $(k) \rightarrow(k)_{\delta}^{\beta}$.

Fact 4.4. If $(k) \rightarrow(k)_{\delta}^{\gamma}$ and $m$ is a subsequence of $k$, then $(m) \rightarrow(m)_{\delta}^{\gamma}$.

Fact 4.5. $(k) \rightarrow(k)^{\delta}$ implies $\kappa \rightarrow(\kappa)^{\delta}$ for every $\kappa \in k$.

Fact 4.6. $(k) \rightarrow(k)^{k}$ implies $(k) \rightarrow(k)_{2^{\delta}}^{d}$ for all $\delta<k(0)$ and $d$ such that $d(\kappa)<\kappa$ for all $\kappa \in k$.

Fact 4.7. If $\gamma$ is a cardinal, then $(k) \rightarrow(k)^{\gamma}$ implies $(k) \rightarrow(k)_{2^{\delta}}^{<\gamma}$, for all $\delta<k(0)$. 
Definition 4.8. A sequence $k$ satisfying $(k) \rightarrow(k)^{k}$ is called a strong polarized partition sequence.

Fact 4.9. (DC) If $(k) \rightarrow(k)^{k}$, then $(k) \rightarrow(k)_{2^{\omega}}^{k}$.

An easy consequence of Fact 4.9 is that one can obtain a set homogeneous simultaneously for countably many partitions of $[k]^{k}$ into 2 . See, for example, [H1].

We prove the following proposition as an example of how a cardinal argument can produce a sequence argument. For a reference, see Theorem 1 of [H1].

Proposition 4.10. If $\gamma<k(0)$ is a cardinal, then $(k) \rightarrow(k)^{\gamma}$ implies $(k) \rightarrow(k)^{<\gamma}$.

Proof. Suppose we are given $f:[k]^{<\gamma} \rightarrow 2$. We define $g:[k]^{\gamma} \rightarrow 2$ as follows. For $W \in[k]^{\gamma}$, consider the sequence of equations:

$$
\begin{aligned}
f\left(\prod_{\kappa \in k}(W(\kappa)(0))\right) & =f\left(\prod_{\kappa \in k}(W(\kappa)(1))\right) \\
f\left(\prod_{\kappa \in k}(W(\kappa)(2), W(\kappa)(3))\right) & =f\left(\prod_{\kappa \in k}(W(\kappa)(4), W(\kappa)(5))\right) \\
& \vdots
\end{aligned}
$$

As $\gamma$ is a cardinal, there is enough room in $\gamma$ to have $\gamma$ equations in the list. Let $g(W)=0$ iff all of the equations are true. Let $C$ be homogeneous for $g$.

Claim. $g^{\prime \prime}[C]^{\gamma}=\{0\}$.

Proof of claim. With simple pigeon-holing, we can construct a $W \in[C]^{\gamma}$ with $g(W)=0$ (start by comparing

$$
f\left(\prod_{\kappa \in k}(C(\kappa)(0))\right), f\left(\prod_{\kappa \in k}(C(\kappa)(1))\right), f\left(\prod_{\kappa \in k}(C(\kappa)(2))\right),
$$

taking two of them, and so on).

To conclude, we claim that $C^{\prime} \in[k]^{k}$ is homogeneous for $f$, where $C^{\prime}(\kappa)=C(\kappa) \uparrow$ $(\kappa \backslash \gamma)$.

Proof of claim. Suppose $\beta<\gamma, X, X^{\prime} \in\left[C^{\prime}\right]^{\beta}$. For each $\kappa \in k$, take enough of $C(\kappa)$ to fill the first $<\beta$ equations in the list and the left side of the $\beta$ th equation. Add to this $X(\kappa)$ plus $\gamma$ much of $C(\kappa)$ above to form $W(\kappa)$, and do the same with $X^{\prime}(\kappa)$ to form $W^{\prime}(\kappa)$. Homogeneity then gives us that $f(W)$ and $f\left(W^{\prime}\right)$ are both equal to the same number, hence equal to each other.

Once again, for working with $\mathrm{AD}$, we will use an alternative characterization of "strong."

Definition 4.11. Let $k$ be a discontinuous $\mu$-sequence of regular cardinals, that is, $k(\alpha)>\sup _{\beta<\alpha} k(\beta)$ for all $\alpha<\mu$. If $\lambda=\sup _{\alpha<\mu} k(\alpha)$, and $F: \lambda \rightarrow \lambda$, we say $F$ is a block function if $\forall \alpha<\mu \forall \beta \in\left[\sup _{\alpha^{\prime}<\alpha} k\left(\alpha^{\prime}\right), k(\alpha)\right) F(\beta) \in\left[\sup _{\alpha^{\prime}<\alpha} k\left(\alpha^{\prime}\right), k(\alpha)\right)$. We say $C \subseteq \lambda$ is block c.u.b. if $\forall \alpha<\mu C \cap k(\alpha)$ is c.u.b. in $k(\alpha)$. Such a sequence has the strong polarized partition property if for every partition $\mathcal{P}$ of the block functions on $\lambda$ into $\{0,1\}$, there is an $S \subseteq \lambda$ such that $\forall \alpha<\lambda|S \cap k(\alpha)|=k(\alpha)$ which is homogeneous for $\mathcal{P}$; that is, $\exists i \in\{0,1\} \forall$ block functions $F: \lambda \rightarrow S \mathcal{P}(F)=i$. 
Easily, this property on $k$ is equivalent to the c.u.b version: for every partition $\mathcal{P}$ of the block functions on $\lambda$ into $\{0,1\}$, there is a block c.u.b $C \subseteq \lambda$ and an $i \in\{0,1\}$ such that $\mathcal{P}(F)=i$ for all block functions $F: \lambda \rightarrow C$ of the correct type.

\section{AD AND PARTITION SEQUENCES}

Throughout this section we assume $\mathrm{AD}+\mathrm{DC}$. We will indicate when we are assuming $\mathbf{V}=L(\mathbb{R})$ as well.

Definition 5.1. A regular cardinal $\kappa$ is very reasonable if there are $\boldsymbol{\Gamma}, \phi$ witnessing $\kappa$ is reasonable, and for all $\beta<\kappa$ there is an $\exists^{\omega^{\omega}} \Delta$ relation $B \subseteq \omega^{\omega} \times \omega^{\omega}$ such that for $x, y \in R_{\beta}, \phi(x)(\beta)<\phi(y)(\beta) \leftrightarrow B(x, y)$.

We note that in all cases where the strong partition relation on $\kappa$ has been shown to hold, $\kappa$ has been shown to be very reasonable.

The main result of this section, Theorem 5.10, implies (cf. Corollary 5.11) that any sequence of very reasonable cardinals of length less than $\sup _{n} \boldsymbol{\delta}_{n}^{1}$ has the desired strong polarized partition property (in fact Corollary 5.12 extends this to longer length sequences). This has the effect of "decoupling" the arguments of this section with those of the (generalized) projective hierarchy analysis. Thus, the arguments of this section do not require knowledge of the projective hierarchy analysis of [J1] or [J2]. For the purposes of this paper, the reader may "abstractly" assume that all of the odd $\delta_{\alpha}^{1}, \alpha<\omega_{1}$, are very reasonable, and that all of the regular cardinals below $\omega_{\omega_{1}}$ are ultrapowers of these by normal measures.

One of the main ideas in the arguments of this section involves the KechrisWoodin theory of generic codes (see $[\mathrm{KeW}]$ ) as well as a category argument involving the generic codes. Similar ideas were used in [J5] to establish the $\boldsymbol{\delta}_{1}^{2}$ supercompactness of the projective ordinals.

We present now a result giving sufficient conditions for a sequence $k$ to have the strong polarized partition property. It is similar in spirit to the "abstract form" of Martin's Proposition 1.10 earlier.

Definition 5.2. Let $k$ be an increasing, discontinuous $\mu$-sequence of regular cardinals. We say the sequence is reasonable if there is a sequence of non-selfdual pointclasses $\left\{\boldsymbol{\Gamma}_{\alpha}\right\}_{\alpha<\mu}$ each closed under $\exists^{\omega^{\omega}}$, and a function $\phi$ with domain $\omega^{\omega}$ satisfying:

1. For all $x \in \omega^{\omega}, \phi(x) \subseteq \lambda \times \lambda$, where $\lambda=\sup _{\alpha<\mu} k(\alpha)$.

2. For every block function $F: \lambda \rightarrow \lambda, \exists x \in \omega^{\omega} \phi(x)=F$.

3. $\forall \alpha<\mu R_{\alpha} \in \Delta_{\alpha+1}$, where $x \in R_{\alpha} \leftrightarrow \forall \alpha^{\prime} \leq \alpha \forall \beta \in\left[\sup _{\alpha^{\prime \prime}<\alpha^{\prime}} k\left(\alpha^{\prime \prime}\right), k\left(\alpha^{\prime}\right)\right)$ $\exists \gamma \in\left[\sup _{\alpha^{\prime \prime}<\alpha^{\prime}} k\left(\alpha^{\prime \prime}\right), k\left(\alpha^{\prime}\right)\right)\left[\phi(x)(\beta, \gamma) \wedge \forall \gamma^{\prime}<\lambda \phi(x)\left(\beta, \gamma^{\prime}\right) \stackrel{\alpha^{\prime \prime}<\alpha^{\prime}}{\rightarrow} \gamma^{\prime}=\gamma\right]$. Thus, $x \in R_{\alpha}$ iff $\phi(x)$ is a block function through $k(\alpha)$.

4. $\forall \alpha<\mu \forall \beta, \gamma \in\left[\sup _{\alpha^{\prime}<\alpha} k\left(\alpha^{\prime}\right), k(\alpha)\right) R_{\alpha, \beta, \gamma} \in \boldsymbol{\Delta}_{\alpha}$, where $x \in R_{\alpha, \beta, \gamma} \leftrightarrow$ $\phi(x)(\beta, \gamma) \wedge \forall \gamma^{\prime}<k(\alpha)\left(\phi(x)\left(\beta, \gamma^{\prime}\right) \rightarrow \gamma^{\prime}=\gamma\right)$.

5. Suppose $\alpha<\mu, \beta \in\left[\sup _{\alpha^{\prime}<\alpha} k\left(\alpha^{\prime}\right), k(\alpha)\right), A \in \exists^{\omega^{\omega}} \boldsymbol{\Delta}_{\alpha}$, and $A \subseteq R_{\alpha, \beta}$, where $x \in R_{\alpha, \beta} \leftrightarrow \exists \gamma<k(\alpha) x \in R_{\alpha, \beta, \gamma}$. Then $\sup \{\phi(x)(\beta): x \in A\}<k(\alpha)$. 
We note that (3) is the new requirement. Likewise, we say the sequence is very reasonable if it is reasonable and $\forall \alpha<\mu \forall \beta \in\left[\sup _{\alpha^{\prime}<\alpha} k\left(\alpha^{\prime}\right), k(\alpha)\right) \exists B \in$ $\exists^{\omega^{\omega}} \boldsymbol{\Delta}_{\alpha} \forall x, y \in R_{\alpha, \beta}[\phi(x)(\beta)<\phi(y)(\beta) \leftrightarrow B(x, y)]$.

We will also use the notation $R_{\alpha}, R_{\alpha, \beta}, R_{\alpha, \beta, \gamma}$ throughout, relative to a coding function $\phi$ for the sequence $\{k(\alpha)\}_{\alpha<\mu}$.

Proposition 5.3. Every reasonable $\mu$-sequence $k$ has the strong polarized partition property.

Proof. Play the integer game where I plays out $x \in \omega^{\omega}$ and II plays out $y \in \omega^{\omega}$. Say $x$ is $(\alpha, \beta)-\operatorname{good}$ if $x \in R_{\alpha, \beta}$, and similarly for $y$. Otherwise, say $x$ is $(\alpha, \beta)-$ bad. If there is a $\beta<\lambda$ such that $x$ or $y$ is $(\alpha, \beta)$-bad (where $\left.\beta \in\left[\sup _{\alpha^{\prime}<\alpha} k\left(\alpha^{\prime}\right), k(\alpha)\right)\right)$, then

II wins provided $x$ is $(\alpha, \beta)$-bad for the least such $\beta$. If both $x, y$ are $(\alpha, \beta)$-good for all $\beta<\lambda$, then $\phi(x), \phi(y)$ are block functions. In this case, II wins iff $\mathcal{P}\left(f_{x, y}\right)=1$, where $f_{x, y}(\beta)=\sup _{\beta^{\prime}<\omega \cdot(\beta+1)} \max \left(\phi(x)\left(\beta^{\prime}\right), \phi(y)\left(\beta^{\prime}\right)\right)$.

Suppose that II wins by $\tau$ (if I wins, the argument is similar but slightly easier). If $\beta, \gamma \in\left[\sup _{\alpha^{\prime}<\alpha} k\left(\alpha^{\prime}\right), k(\alpha)\right)$, define $x \in S_{\alpha, \beta, \gamma} \leftrightarrow\left(\forall \alpha^{\prime}<\alpha x \in R_{\alpha^{\prime}}\right) \wedge\left(\forall \beta^{\prime} \leq \beta \exists \gamma^{\prime} \leq\right.$ $\left.\gamma x \in R_{\alpha, \beta^{\prime}, \gamma^{\prime}}\right)$. Let $g(\beta, \gamma)=\sup \left\{\phi(\tau(x))(\beta): x \in S_{\alpha, \beta, \gamma}\right\}$. Since $S_{\alpha, \beta, \gamma} \in \boldsymbol{\Delta}_{\alpha}$, and $A=\tau\left[S_{\alpha, \beta, \gamma}\right] \subseteq R_{\alpha, \beta}$ is in $\exists^{\omega^{\omega}} \boldsymbol{\Delta}_{\alpha}, g(\beta, \gamma)<k(\alpha)$. Let $C \subseteq \lambda$ be block c.u.b. and closed under $g$, and let $C^{\prime}$ be the limit points of $C$. Suppose $F: \lambda \rightarrow C^{\prime}$ is a block function of the correct type. There is an increasing block function $f: \lambda \rightarrow C$ such that $\forall \alpha<\mu \forall \beta \in\left[\sup _{\alpha^{\prime}<\alpha} k\left(\alpha^{\prime}\right), k(\alpha)\right) F(\beta)=\sup _{\beta^{\prime}<\omega \cdot(\beta+1)} f\left(\beta^{\prime}\right)$. Let $\phi(x)=f$, and $y=\tau(x)$. From the definition of $C, \phi(y)(\beta)<\phi(x)(\beta+1)$ for all $\beta<\lambda$, and thus $F=f_{x, y}$. Hence, $\mathcal{P}(F)=1$.

We recall some results in the abstract theory of pointclasses which we will need below. The reader not wishing to trudge through the pointclass arguments of the next several lemmas (which assumes some familiarity with [St1]) may skip to Proposition 5.7, and strengthen the definitions of reasonable, very reasonable, by requiring the additional closure properties of $\boldsymbol{\Gamma}, \boldsymbol{\Delta}$.

First, if $\boldsymbol{\Gamma}$ is a non-selfdual pointclass closed under $\exists^{\omega^{\omega}}$ or $\forall^{\omega^{\omega}}$, then either $\operatorname{PWO}(\boldsymbol{\Gamma})$ or $\operatorname{PWO}(\check{\boldsymbol{\Gamma}})$. This is shown at the end of [St1], and generalizes a result of [KeSoSt]. Secondly, if $\boldsymbol{\Gamma}$ is a non-selfdual pointclass closed under $\exists^{\omega^{\omega}}$ and PWO $(\boldsymbol{\Gamma})$, then $\boldsymbol{\Gamma}$ is closed under well-ordered unions. If $\boldsymbol{\Gamma}$ is closed under $\forall^{\omega^{\omega}}$ as well, this is Theorem 1.1 of [JMa]. If $\boldsymbol{\Gamma}$ is closed under countable intersections (note: $\boldsymbol{\Gamma}$ is closed under countable unions), then this follows from Lemma 2.4.1 of [KeSoSt]. This includes the case where $\boldsymbol{\Gamma}=\exists^{\omega^{\omega}} \boldsymbol{\Gamma}^{\prime}$, for some non-selfdual $\boldsymbol{\Gamma}^{\prime}$ closed under $\forall^{\omega^{\omega}}$. The only remaining case (cf. the analysis at the end of [St1]) is when $\boldsymbol{\Gamma}$ is at the base of a type I hierarchy. That is, $\boldsymbol{\Delta}=\boldsymbol{\Gamma} \cap \check{\boldsymbol{\Gamma}}$ is closed under $\exists^{\omega^{\omega}}, \forall^{\omega^{\omega}}$, and $\boldsymbol{\Gamma}$ is the collection of countable unions of sets in $\boldsymbol{\Delta}$. We sketch the proof in this case.

Towards a contradiction, let $\kappa$ be the least cardinal so that $\bigcup_{\kappa} \boldsymbol{\Gamma} \nsubseteq \boldsymbol{\Gamma}$. Thus, $\kappa$ is regular and by the Coding Lemma $\kappa \geq o(\boldsymbol{\Delta}$ ), the Wadge ordinal of $\boldsymbol{\Delta}$ (which is also the supremum of the $\boldsymbol{\Delta}$ pre-well-orderings; see [KeSoSt $])$. Also, $\kappa \neq o(\boldsymbol{\Delta})$ as $\operatorname{cof}(o(\boldsymbol{\Delta}))=\omega$. Thus, $\kappa>o(\boldsymbol{\Delta})$. Let $\boldsymbol{\Gamma}_{1}=\exists^{\omega^{\omega}} \check{\boldsymbol{\Gamma}}$. By Wadge, $\check{\boldsymbol{\Gamma}} \subseteq \bigcup_{\kappa} \boldsymbol{\Gamma}$, and so $\boldsymbol{\Gamma}_{1} \subseteq \bigcup_{\kappa} \boldsymbol{\Gamma}$. Using the regularity of $\kappa$, let $\left\langle A_{\alpha}: \alpha<\kappa\right\rangle$ be a strictly increasing $\kappa$ sequence of sets in $\boldsymbol{\Gamma}$ whose union $A$ is in $\check{\Gamma}$. Let $B=\left\{x: S_{x} \subseteq A\right\}$, where $S$ is universal $\boldsymbol{\Sigma}_{1}^{1} . \quad B \in \check{\boldsymbol{\Gamma}}$ as $\check{\boldsymbol{\Gamma}}$ is closed under $\forall^{\omega^{\omega}}$ and $\vee$. Let $B=\bigcup_{\alpha<\kappa} B_{\alpha}$, 
where $B_{\alpha} \in \boldsymbol{\Gamma}$. If we replace $A_{\alpha}$ by $\left\{y: \exists x \in B_{\alpha}\left(y \in S_{x}\right)\right\}$, then the $A_{\alpha}$ form a $\boldsymbol{\Sigma}_{1}^{1}$-bounded union of $\boldsymbol{\Gamma}$ sets; that is, every $\boldsymbol{\Sigma}_{1}^{1}$ subset of $A$ is contained in some $A_{\alpha}$. Let $C$ be a universal $\boldsymbol{\Gamma}$ set. Play the game where I plays $x$, II plays $y, z$, and II wins iff $x \in A \rightarrow \exists \alpha>|x|\left(C_{y}=A_{\alpha} \wedge z \in A_{\alpha}-\bigcup_{\beta<\alpha} A_{\beta}\right)$, where $|x|$ is the least $\alpha<\kappa$ such that $x \in A_{\alpha}$. By $\boldsymbol{\Sigma}_{1}^{1}$-boundedness, II wins, say by $\tau$. Define $x \prec y$ iff $x, y \in A \wedge \tau(y)_{1} \notin C_{\tau(x)_{0}}$. Thus, $\prec$ is a $\check{\Gamma}$ pre-well-ordering of length $\kappa$. By the Coding Lemma, then, $\bigcup_{\kappa} \boldsymbol{\Gamma} \subseteq \boldsymbol{\Gamma}_{1}$, and hence $\bigcup_{\kappa} \boldsymbol{\Gamma}=\boldsymbol{\Gamma}_{1}$. Now, $\boldsymbol{\Delta}_{1}=\boldsymbol{\Gamma}_{1} \cap \check{\boldsymbol{\Gamma}}_{1}$ is clearly also not closed under $\kappa$ unions, and any $\kappa$ union of sets in $\boldsymbol{\Delta}_{1}$ is in $\boldsymbol{\Gamma}_{1}$. Thus, $\boldsymbol{\Gamma}_{1}=\bigcup_{\kappa^{\prime}} \boldsymbol{\Delta}_{1}$, where $\kappa^{\prime} \leq \kappa$ is least such that $\boldsymbol{\Delta}_{1}$ is not closed under $\kappa^{\prime}$ unions. This, however, shows $\operatorname{PWO}\left(\boldsymbol{\Gamma}_{1}\right)$, a contradiction, since by periodicity $\operatorname{PWO}\left(\check{\boldsymbol{\Gamma}}_{1}\right)$ (this last part is an argument of Martin).

The next several lemmas develop the abstract theory of reasonable pointclasses.

Lemma 5.4. Suppose $\kappa_{1}<\kappa_{2}$ are reasonable with corresponding pointclasses $\boldsymbol{\Gamma}_{1}, \boldsymbol{\Gamma}_{2}$. Then $\exists^{\omega^{\omega}} \check{\boldsymbol{\Gamma}}_{1} \subseteq \boldsymbol{\Delta}_{2}=\boldsymbol{\Gamma}_{2} \cap \check{\boldsymbol{\Gamma}}_{2}$.

Proof. If not, then $\boldsymbol{\Gamma}_{2}=\exists^{\omega}{ }^{\omega} \check{\boldsymbol{\Gamma}}_{1}$. Then either $\boldsymbol{\Gamma}_{1}$ or $\boldsymbol{\Gamma}_{2}$ has the pre-well-ordering property. Assume first $\operatorname{PWO}\left(\boldsymbol{\Gamma}_{1}\right)$. Thus, $\boldsymbol{\Gamma}_{1}$ is closed under well-ordered unions. For $\gamma<\kappa_{1}$, consider $R_{0, \gamma}=\{x: \phi(x)(0)$ is defined and $\leq \gamma\}$. $\phi$ here refers to the coding for $\kappa_{1}$. By reasonableness, each $R_{0, \gamma} \in \Delta_{1}$, and so $R=\bigcup_{\gamma<\kappa_{1}} R_{0, \gamma} \in \boldsymbol{\Gamma}_{1}$. This contradicts reasonableness if $\boldsymbol{\Gamma}_{1}=\exists^{\omega^{\omega}} \boldsymbol{\Delta}_{1}$. So assume $\boldsymbol{\Delta}_{1}$ is closed under real quantifiers. Note that $\boldsymbol{\Gamma}_{1}$ is not closed under $\forall^{\omega}$ by assumption. By $\operatorname{PWO}\left(\boldsymbol{\Gamma}_{1}\right)$, an inspection of the hierarchy analysis (cf. [St1]) shows that $\boldsymbol{\Gamma}_{1}$ is the base of a type I hierarchy, that is, $\boldsymbol{\Gamma}_{1}=\bigcup_{\omega} \boldsymbol{\Delta}_{1}$. Since $\operatorname{cof}(o(\boldsymbol{\Delta}))=\omega$, we must have $o\left(\boldsymbol{\Delta}_{1}\right) \neq \kappa_{1}$. We cannot have $\kappa_{1}<o\left(\boldsymbol{\Delta}_{1}\right)$, as then (by the Coding Lemma and the fact that cofinally many of the $R_{0, \gamma}$ lie in some fixed $\left.\boldsymbol{\Lambda} \subsetneq \boldsymbol{\Delta}_{1}\right) R \in \boldsymbol{\Delta}_{1}$, a contradiction to reasonableness. So $o\left(\boldsymbol{\Delta}_{1}\right)<\kappa$. Since $\kappa$ is regular, there is a non-selfdual $\boldsymbol{\Lambda} \subseteq \boldsymbol{\Delta}_{1}$ closed under $\exists^{\omega^{\omega}}$ with $\operatorname{PWO}(\boldsymbol{\Lambda})$, and such that $\kappa$ many of the $R_{0, \gamma}$ are in $\boldsymbol{\Lambda}$. Since $\boldsymbol{\Lambda}$ is closed under well-ordered unions, $R \in \boldsymbol{\Lambda}$ (note: the $R_{0, \gamma}$ form an increasing sequence), a contradiction to reasonableness. Assume now $\operatorname{PWO}\left(\boldsymbol{\Gamma}_{2}\right)$. We may assume $\boldsymbol{\Delta}_{2}$ is closed under real quantification, arguing as above. This, however, contradicts $\boldsymbol{\Gamma}_{2}=\exists^{\omega^{\omega}} \check{\boldsymbol{\Gamma}}_{1}$.

Lemma 5.5. Let $\kappa$ be reasonable, with corresponding pointclass $\boldsymbol{\Gamma}$, and map $\phi$. Then $\kappa$ is the supremum of the $\boldsymbol{\Delta}$ pre-well-orderings of the reals. Furthermore, $\boldsymbol{\Gamma}$ is uniquely determined from $\kappa$. Finally, $\operatorname{PWO}(\check{\boldsymbol{\Gamma}})$ if $\boldsymbol{\Gamma}$ is not closed under real quantification.

Proof. Let $\boldsymbol{\Delta}=\boldsymbol{\Gamma} \cap \check{\boldsymbol{\Gamma}}$, and first assume $\boldsymbol{\Delta}$ is closed under real quantification. Thus, $o(\boldsymbol{\Delta})$ is the supremum of the $\boldsymbol{\Delta}$ pre-well-orderings (cf. [KeSoSt]). We cannot have $\kappa<o(\boldsymbol{\Delta})$, as then by the Coding Lemma there is an unbounded subset of $R_{0}=\bigcup_{\gamma<\kappa} R_{0, \gamma}$ in $\boldsymbol{\Delta}$, violating the reasonableness of $\kappa$. If $\kappa>o(\boldsymbol{\Delta})$, then by regularity of $\kappa, \kappa$ many of the $R_{0, \gamma}$ lie in some non-selfdual pointclass $\boldsymbol{\Lambda} \subseteq \boldsymbol{\Delta}$ closed under $\exists^{\omega^{\omega}}$ with $\operatorname{PWO}(\boldsymbol{\Lambda})$. Then $R_{0} \in \boldsymbol{\Lambda} \subseteq \boldsymbol{\Delta}$, violating reasonableness. Thus, $\kappa=o(\boldsymbol{\Delta})$. In particular, $o(\boldsymbol{\Delta})$ is regular, so $\boldsymbol{\Gamma}$ cannot be the base of a type I or II hierarchy. If $\boldsymbol{\Gamma}$ is not closed under real quantification, then from [St1] we have $\operatorname{PWO}(\check{\boldsymbol{\Gamma}})$, as $\boldsymbol{\Gamma}$ is closed under $\exists^{\omega^{\omega}}$ ([St1] shows that PWO falls on the side closed under $\forall^{\omega}$ at this level). 
Assume now $\boldsymbol{\Gamma}=\exists^{\omega}{ }^{\omega} \boldsymbol{\Delta}$. We cannot have $\operatorname{PWO}(\boldsymbol{\Gamma})$, as then $\boldsymbol{\Gamma}$ is closed under well-ordered unions, so $R_{0} \in \exists^{\omega^{\omega}} \boldsymbol{\Delta}$, violating reasonableness. Thus, $\operatorname{PWO}(\check{\boldsymbol{\Gamma}})$. Let $\delta$ be the supremum of the lengths of the $\boldsymbol{\Delta}$ pre-well-orderings.

Suppose first that $\boldsymbol{\Gamma}=\exists^{\omega^{\omega}} \boldsymbol{\Gamma}_{-}$for some non-selfdual $\boldsymbol{\Gamma}_{-}$closed under $\forall^{\omega}$ (this covers all cases except for $\boldsymbol{\Gamma}=\boldsymbol{\Gamma}_{-} \vee \check{\boldsymbol{\Gamma}}_{-}$where $\boldsymbol{\Gamma}_{-}$is closed under real quantification). The Coding Lemma again shows $\delta \leq \kappa$. Suppose towards a contradiction that $\delta<\kappa$. Let $\boldsymbol{\Gamma}_{1}=\exists^{\omega^{\omega}} \check{\boldsymbol{\Gamma}}$, so $\boldsymbol{\Gamma}_{1}$ is closed under well-ordered unions. By $\operatorname{PWO}(\check{\boldsymbol{\Gamma}})$, $\bigcup_{\delta} \boldsymbol{\Gamma} \neq \boldsymbol{\Gamma}$, and hence $\bigcup_{\delta} \boldsymbol{\Gamma}=\boldsymbol{\Gamma}_{1}$. In particular, $R_{0}=\bigcup_{\alpha<\delta} A_{\alpha}$, where each $A_{\alpha} \in \boldsymbol{\Gamma}$. One of the $A_{\alpha}$ must be unbounded in $k(\alpha)$ (with respect to the sequence $R_{0, \gamma}$ ), contradicting reasonableness.

In the remaining case, $\boldsymbol{\Gamma}=\boldsymbol{\Gamma}_{-} \vee \check{\boldsymbol{\Gamma}}_{-}$where $\boldsymbol{\Gamma}_{-}$is closed under real quantification. We will show this case cannot occur. Without loss of generality assume $\operatorname{PWO}\left(\boldsymbol{\Gamma}_{-}\right)$. First, the Coding Lemma easily shows that $\kappa \geq \delta_{-}=$the supremum of the $\boldsymbol{\Delta}_{-}$ pre-well-orderings. Also, we cannot have $\kappa=\delta_{-}$. If so, then by the Coding Lemma we could get a $\boldsymbol{\Gamma}_{-}$set $A \subseteq \bigcup_{\gamma<\kappa} R_{0, \gamma}$ which is unbounded, that is, not contained in any $R_{0, \gamma}$. This contradicts reasonableness. So assume $\kappa>\delta_{-}$. Let $\boldsymbol{\Gamma}_{1}=\exists^{\omega^{\omega}}\left(\boldsymbol{\Gamma}_{-} \wedge \check{\boldsymbol{\Gamma}}_{-}\right)$. From [St1] we have $\operatorname{PWO}(\check{\boldsymbol{\Gamma}})$ and thus $\operatorname{PWO}\left(\boldsymbol{\Gamma}_{1}\right)$. Hence, $\boldsymbol{\Gamma}_{1}$ is closed under well-ordered unions. In particular, $R_{0}=\bigcup_{\gamma<\kappa} R_{0, \gamma} \in \boldsymbol{\Gamma}_{1}$. Also, we easily have $\boldsymbol{\Gamma}_{1}=\bigcup_{\delta_{-}} \check{\boldsymbol{\Gamma}}_{-}$. Write $R_{0}=\bigcup_{\alpha<\delta_{-}} A_{\alpha}$ where $A_{\alpha} \in \check{\boldsymbol{\Gamma}}_{-}$. By the regularity of $\kappa$, some $A_{\alpha}$ is unbounded in the $R_{0, \gamma}$ union. Since $A_{\alpha} \in \check{\boldsymbol{\Gamma}}_{-} \subseteq \boldsymbol{\Delta}$, this contradicts reasonableness.

It remains to show that $\kappa$ determines $\boldsymbol{\Gamma}$. Suppose $\boldsymbol{\Gamma}_{1}, \boldsymbol{\Gamma}_{2}$ both witness the reasonableness of $\kappa$, and suppose $\boldsymbol{\Gamma}_{1} \subseteq \boldsymbol{\Gamma}_{2}$. If $\boldsymbol{\Gamma}_{1}$ is closed under countable intersections but not real quantification, then by standard arguments using $\operatorname{PWO}(\check{\boldsymbol{\Gamma}})$ there is a $\check{\boldsymbol{\Gamma}}_{1}$ pre-well-ordering of length $\kappa=$ the supremum of the lengths of the $\boldsymbol{\Delta}_{1}$ prewell-orderings. Since $\check{\boldsymbol{\Gamma}}_{1} \subseteq \boldsymbol{\Delta}_{2}$, we contradict $\kappa$ being the supremum of the $\boldsymbol{\Delta}_{2}$ pre-well-orderings. In the remaining cases, $\boldsymbol{\Delta}_{1}$ is closed under real quantification. If $\boldsymbol{\Gamma}_{1}$ is not closed under real quantifiers, then by Lemma 5.4 (the proof still holds when $\left.\kappa_{1}=\kappa_{2}\right) \exists^{\omega^{\omega}} \check{\boldsymbol{\Gamma}}_{1} \subseteq \boldsymbol{\Delta}_{2}$. However, there is an $\exists^{\omega^{\omega}} \check{\boldsymbol{\Gamma}}_{1}$ pre-well-ordering of length $\kappa$, a contradiction. Finally, if $\boldsymbol{\Gamma}_{1}$ is closed under real quantifiers, then there is a $\boldsymbol{\Gamma}_{1} \wedge \check{\boldsymbol{\Gamma}}_{1}$ pre-well-ordering of length $\kappa$. Since $\operatorname{PWO}\left(\exists^{\omega^{\omega}}\left(\boldsymbol{\Gamma}_{1} \wedge \check{\boldsymbol{\Gamma}}_{1}\right)\right)$, we must have $\exists^{\omega^{\omega}}\left(\boldsymbol{\Gamma}_{1} \wedge \check{\boldsymbol{\Gamma}}_{1}\right) \subseteq \boldsymbol{\Delta}_{2}$, a contradiction.

Lemma 5.6. Let $\kappa$ be reasonable with corresponding pointclass $\boldsymbol{\Gamma}$ and map $\phi$. Then $\boldsymbol{\Gamma}$ is closed under countable unions and intersections. Furthermore, $\boldsymbol{\Delta}$ is closed under $<\kappa$ length unions and intersections.

Proof. If $\boldsymbol{\Gamma}=\exists^{\omega^{\omega}} \boldsymbol{\Gamma}_{0}$ for some $\boldsymbol{\Gamma}_{0}$ closed under $\forall^{\omega^{\omega}}$, easily $\boldsymbol{\Gamma}$ is closed under countable intersections. As observed in the previous lemma, for the remaining cases we have $\boldsymbol{\Delta}$ closed under real quantification. We must show $\check{\boldsymbol{\Gamma}}$ is closed under countable unions. From Theorem 2.2 of [St1], it suffices to show $\check{\boldsymbol{\Gamma}}$ is closed under finite unions. Let $\boldsymbol{\Gamma}^{\prime}$ be the collection of $\boldsymbol{\Sigma}_{1}^{1}$-bounded unions of $\boldsymbol{\Delta}$ sets of length $\kappa=o(\boldsymbol{\Delta})$. Let $\Gamma^{\prime \prime}$ likewise be the collection of $\boldsymbol{\Delta}$-bounded unions. Clearly $\boldsymbol{\Gamma}^{\prime \prime} \subseteq \boldsymbol{\Gamma}^{\prime}$. The proof of Theorem 3.1 of [St1] shows that $\boldsymbol{\Gamma}^{\prime}=\check{\boldsymbol{\Gamma}}$. By reasonableness, the $R_{0, \gamma}, \gamma<\kappa$, form a $\boldsymbol{\Delta}$-bounded union of $\boldsymbol{\Delta}$ sets whose union $R_{0}$ is not in $\boldsymbol{\Delta}$. Thus, $\boldsymbol{\Gamma}^{\prime \prime}=\boldsymbol{\Gamma}^{\prime}$. We now follow the argument of Theorem 3.2 of [St1]. Let $A, B \in \check{\boldsymbol{\Gamma}}$, and by $\operatorname{RED}(\check{\boldsymbol{\Gamma}})$ we may assume $A \cap B=\emptyset$. Write $A=\bigcup_{\alpha<\kappa} A_{\alpha}, B=\bigcup_{\alpha<\kappa} B_{\alpha}$ as $\boldsymbol{\Delta}$-bounded unions. It suffices to show the union $A \cup B=\bigcup_{\alpha<\kappa}\left(A_{\alpha} \cup B_{\alpha}\right)$ is $\boldsymbol{\Sigma}_{1}^{1}$-bounded. Let $S \subseteq A \cup B$ be $\boldsymbol{\Sigma}_{1}^{1}$. $S \cap A \in \check{\boldsymbol{\Gamma}}$ as $\check{\boldsymbol{\Gamma}}$ is closed under conjunction. Also, $S \cap A=S \cap B^{c} \in \boldsymbol{\Gamma}$, 
as $\boldsymbol{\Gamma}$ is closed under conjunction with $\boldsymbol{\Sigma}_{1}^{1}$ sets as Steel points out (the union of a $\check{\boldsymbol{\Gamma}}$ set and a $\Pi_{1}^{1}$ set can be written in the form $\forall z(U \vee \check{\boldsymbol{\Gamma}})$ where $U$ is open, and $\check{\boldsymbol{\Gamma}}$ is easily seen to be closed under unions with open sets). Thus, $S \cap A \in \boldsymbol{\Delta}$, and likewise $S \cap B \in \boldsymbol{\Delta}$. By $\boldsymbol{\Delta}$-boundedness, $S \subseteq A_{\alpha} \cup B_{\alpha}$ for some $\alpha<\kappa$.

The closure of $\boldsymbol{\Delta}$ under $<\kappa$ unions follows easily from the Coding Lemma and the regularity of $\kappa$ if $\boldsymbol{\Delta}$ is closed under quantifiers. Otherwise, Martin's argument shows $\operatorname{PWO}(\boldsymbol{\Gamma})$, a contradiction to Lemma 5.5.

The next is a special case of Theorem 5.10 (with reasonable replaced by very reasonable), but is simple enough to present separately.

Proposition 5.7. Let $k$ be an $\omega$-sequence of reasonable cardinals with corresponding pointclasses $\boldsymbol{\Gamma}_{i}$. Then $k$ has the strong polarized partition property.

Proof. By countable choice, let $\phi_{i}$, along with the $\boldsymbol{\Gamma}_{i}$, witness the reasonableness of $k(i)$. We may identify $k(i)$ with $\left[\sup _{i^{\prime}<i} k\left(i^{\prime}\right), k(i)\right)$, and thus $\phi_{i}(x) \subseteq\left[\sup _{i^{\prime}<i} k\left(i^{\prime}\right), k(i)\right) \times$ $\left[\sup _{i^{\prime}<i} k\left(i^{\prime}\right), k(i)\right)$. With this convention, let $\phi(x)=\bigcup_{i} \phi_{i}\left(x_{i}\right)$, where $x \rightarrow\left(x_{0}, x_{1}, \ldots\right)$ is a recursive bijection. Properties 1,2 are immediate, and $3,4,5$ follow easily provided we show that $R_{i} \in \boldsymbol{\Delta}_{i+1}$ for all $i$. Now $x \in R_{i}$ iff $x \in R_{i-1}$ and $\forall \beta<k(i)$ $\exists \gamma<k(i) x \in R_{i, \beta, \gamma}$. By induction, $R_{i-1} \in \boldsymbol{\Delta}_{i} \subseteq \boldsymbol{\Delta}_{i+1}$. Also, each $R_{i, \beta, \gamma} \in \boldsymbol{\Delta}_{i}$.

Assume first that $\boldsymbol{\Gamma}_{i}$ is closed under real quantifiers, and without loss of generality assume $\operatorname{PWO}(\boldsymbol{\Gamma})$ (the reasonableness hypothesis in this case is symmetric between $\boldsymbol{\Gamma}_{i}$ and $\left.\check{\boldsymbol{\Gamma}}_{i}\right)$. Thus, $\boldsymbol{\Gamma}_{i}$ is closed under well-ordered unions, and hence $R_{i}$ is a $\kappa$ intersection of $\boldsymbol{\Gamma}_{i}$ sets. It suffices to show that a $\kappa$ union of $\check{\boldsymbol{\Gamma}}$ sets is in $\boldsymbol{\Delta}_{i+1}$. Let $\boldsymbol{\Gamma}^{*}=\exists^{\omega^{\omega}}(\boldsymbol{\Gamma} \wedge \check{\boldsymbol{\Gamma}})$. Then $\operatorname{PWO}\left(\boldsymbol{\Gamma}^{*}\right)$ (see [St1]), so $\boldsymbol{\Gamma}^{*}$ is closed under well-ordered unions. In the proof of Lemma 5.5 we showed $\boldsymbol{\Gamma}^{*}=\boldsymbol{\Gamma}_{i+1}$ is impossible, and thus $\Gamma^{*} \subseteq \Delta_{i+1}$.

Assume now that $\boldsymbol{\Gamma}_{i}$ is not closed under real quantification, so $\mathrm{PWO}\left(\check{\boldsymbol{\Gamma}}_{i}\right)$. Let $\boldsymbol{\Gamma}_{i}^{*}=\exists^{\omega^{\omega}} \check{\boldsymbol{\Gamma}}_{i}$, so $\mathrm{PWO}\left(\boldsymbol{\Gamma}_{i}^{*}\right)$ and $\boldsymbol{\Gamma}_{i}^{*}$ is closed under well-ordered unions. It suffices to show that $\bigcup_{k(i)} \check{\boldsymbol{\Gamma}}_{i}^{*} \subseteq \boldsymbol{\Delta}_{i+1}$. First note that there is a $\boldsymbol{\Gamma}_{i}^{*}$ pre-well-ordering of length $k(i)$. This follows from the existence of a $k(i)$ increasing sequence of sets in $\boldsymbol{\Delta}_{i}$ and the closure of $\boldsymbol{\Gamma}_{i}^{*}$ under well-ordered unions. From the Coding Lemma, it follows that $\bigcup_{k(i)} \check{\boldsymbol{\Gamma}}_{i}^{*} \subseteq \exists{ }^{\omega} \check{\boldsymbol{\Gamma}}_{i}^{*}$. If equality holds here, then easily $\operatorname{PWO}\left(\exists^{\omega^{\omega}} \check{\boldsymbol{\Gamma}}_{i}^{*}\right)$, a contradiction. This gives the desired result unless $\boldsymbol{\Gamma}_{i+1}=\boldsymbol{\Gamma}_{i}^{*}$. This, however, contradicts Lemma 5.4.

It is natural to ask how far Proposition 5.7 can be extended. That is, for which $\alpha \leq \Theta$ can we show that an increasing, discontinuous $\alpha$ sequence of reasonable cardinals has the strong polarized partition property?

We assume $\mathbf{V}=L(\mathbb{R})$ for the remainder of this section, though $\mathrm{AD}+\mathrm{DC}+$ "within scales" would suffice for many of the results (e.g., Corollaries 5.12 and 5.13). Recall $\boldsymbol{\delta}_{1}^{2}$ is the largest Suslin cardinal in $L(\mathbb{R})$. If $\alpha<\boldsymbol{\delta}_{1}^{2}$, let $s(\alpha)$ be the least Suslin cardinal $>\alpha$ such that the class of $s(\alpha)$-Suslin sets is of the form $\boldsymbol{\Sigma}_{1}\left(J_{\beta}(\mathbb{R})\right)$ for some $\beta$ beginning a $\boldsymbol{\Sigma}_{1}$-gap. Thus, we "skip over" the Suslin cardinals ocurring at the end of a gap. Let $S_{s(\alpha)}$ be the pointclass of $s(\alpha)$-Suslin sets.

The point here is that the construction of scales for such pointclasses is uniform, according to the analysis of [St2]. That is, there is a function which assigns to each $\alpha<\delta_{1}^{2}$ a universal $S_{s(\alpha)}$ set $P_{s(\alpha)}$ and an $S_{s(\alpha)}$-scale $\phi_{n}^{s(\alpha)}$ on $P_{s(\alpha)}$ with $\phi_{0}^{s(\alpha)}$ 
onto $s(\alpha)$. For $x \in P_{s(\alpha)}$, we let $|x|_{s(\alpha)}$ abbreviate $\phi_{0}^{s(\alpha)}(x)$, and similarly for other scales.

Definition 5.8. Let $k$ be an increasing, discontinuous $\mu$-sequence of reasonable cardinals with corresponding pointclasses $\boldsymbol{\Gamma}_{\alpha}$ for each $k(\alpha)$. We say that $k$ is proper if $\forall \alpha<\mu\left(\forall^{\omega^{\omega}} S_{s(\alpha)} \subseteq \boldsymbol{\Gamma}_{\alpha}\right)$.

In general, properness requires the $k(\alpha)$ to be sufficiently spread out, although the requirement is trivial for short sequences. For example, an $\omega_{1}$ sequence is proper provided only $k(0) \geq \boldsymbol{\delta}_{3}^{1}$.

We recall some facts from the Kechris-Woodin theory of generic codes that we will need for the proof of our main theorem. Fix for the moment $\alpha_{0}<\delta_{1}^{2}$, and thus $P_{s\left(\alpha_{0}\right)}, \phi_{n}=\phi_{n}^{s\left(\alpha_{0}\right)}$. The main lemma of [KeW] says there is a Lipschitz continuous function $G: s\left(\alpha_{0}\right)^{\omega} \rightarrow \omega^{\omega}$ such that $\forall \vec{t}=\left(\alpha, \beta_{0}, \beta_{1}, \ldots\right) \in s\left(\alpha_{0}\right)^{\omega} \forall n \in$ $\omega G(\vec{t})_{n} \in P_{s\left(\alpha_{0}\right)}$ (where $y \rightarrow\left(y_{0}, y_{1}, \ldots\right)$ is a recursive bijection). Furthermore, if $\vec{t}$ enumerates an honest set $T$, then $\forall n \phi_{0}\left(G(\vec{t})_{n}\right)=\beta_{n}$. Recall $T \in \mathcal{P}_{\omega_{1}}\left(s\left(\alpha_{0}\right)\right)$ is honest if $\forall \beta \in T \exists x \in P_{s\left(\alpha_{0}\right)}\left[\phi_{0}(x)=\beta \wedge \forall n \phi_{n}(x) \in T\right]$.

Recall also that $\omega_{1}$ is $\boldsymbol{\delta}_{1}^{2}$-supercompact (Solovay), that is, there is a fine normal measure on $\mathcal{P}_{\omega_{1}}\left(\boldsymbol{\delta}_{1}^{2}\right)$. By Woodin [W], this measure is in fact unique. We write $\forall^{*} T \in \mathcal{P}_{\omega_{1}}\left(s\left(\alpha_{0}\right)\right)$ to mean almost all with respect to this measure. By normality, almost all $T$ are honest.

Fix now $\mu \leq \delta_{1}^{2}$ and a proper $\mu$-sequence $k$. The proof of the generic Coding Lemma of $[\mathrm{KeW}]$ shows that the coding function $G$ is obtained uniformly in the set $P_{s\left(\alpha_{0}\right)}$ and the scale $\phi_{n}^{s\left(\alpha_{0}\right)}$ on $P_{s\left(\alpha_{0}\right)}$ for $\alpha_{0}<\mu$. G is obtained as a winning strategy in a Suslin, co-Suslin ordinal game on $s\left(\alpha_{0}\right)$. The trees witnessing that the game is Suslin, co-Suslin are obtained uniformly from $P_{s\left(\alpha_{0}\right)}, \phi_{n}^{s\left(\alpha_{0}\right)}$, and the third periodicity argument of [Mo1] shows $G$ is obtained uniformly from these trees.

Thus, we have a Lipschitz continuous function $G$ satisfying:

1. $\forall \alpha<\mu \forall\left(\beta_{1}, \beta_{2}, \ldots\right) \in s(\alpha)^{\omega} G(\vec{t}) \in P_{s(\alpha)}$, where $\vec{t}=\left(\alpha, \beta_{1}, \beta_{2}, \ldots\right)$.

2. If $\left(\alpha, \beta_{1}, \beta_{2}, \ldots\right) \in s(\alpha)^{\omega}$ enumerates an honest set (with respect to $P_{s(\alpha)}$, $\left.\phi_{n}^{s(\alpha)}\right)$, then $\forall n \phi_{0}^{s(\alpha)}\left(G(\vec{t})_{n}\right)=\beta_{n-1}$ (with $\left.\beta_{0}=\alpha\right)$.

We fix this function $G$ for the remainder of this section.

Lemma 5.9. There is a Lipschitz continuous function $F: \mu^{\omega} \rightarrow \omega^{\omega}$ such that $\forall \alpha<\mu \forall \vec{\beta} \in s(\alpha)^{\omega}$, if $\vec{t}=(\alpha, \vec{\beta})$ enumerates an honest set then $u=F(\vec{t})$ codes the following:

1. $A \boldsymbol{\Gamma}_{\alpha}$ universal set $A_{u}$.

2. A map $\phi_{u}$ such that $\left(k(\alpha), \boldsymbol{\Gamma}_{\alpha}, \phi_{u}\right)$ is reasonable. We let $R_{\alpha}^{u}, R_{\alpha, \beta}^{u}, R_{\alpha, \beta, \gamma}^{u}$ denote the corresponding sets.

The exact manner in which $u$ codes these objects is not important, say by the Coding Lemma relative to a fixed set of high Wadge degree.

Proof. Consider the game where I plays $\alpha<\mu, \beta_{1}, \beta_{3}, \ldots \in s(\alpha)$, and II plays $\beta_{2}, \beta_{4}, \ldots \in s(\alpha)$ and also $u(0), u(1), \ldots$ building $u \in \omega^{\omega}$. II wins in case 1,2 above hold, where in place of $\alpha$ we use $\phi_{0}^{s(\alpha)}\left(G(\vec{t})_{0}\right)$.

First note that the game is determined by a variation of the usual reflection argument. The payoff set for II is of the form $(\alpha, \vec{\beta}) \in A$ iff $(\alpha, G(\vec{t})) \in B$, where $B \subseteq \mu \times \omega^{\omega}$, and $G: \mu^{\omega} \rightarrow \omega^{\omega}$ is continuous. If the game is undetermined, then 
there is a $\delta<\delta_{1}^{2}$ and $A^{\prime}, B^{\prime}, G^{\prime}, \mu^{\prime} \in J_{\delta}(\mathbb{R})$ such that $J_{\delta}(\mathbb{R})=($ enough of $Z F)+$ ( $A^{\prime}$ is not determined). However, if $B_{\alpha}^{\prime}=\left\{x:(\alpha, x) \in B^{\prime}\right\}$, then the $B_{\alpha}^{\prime}$ are uniformly $\boldsymbol{\Sigma}_{1}\left(J_{\delta^{\prime}}(\mathbb{R})\right.$ ) for some $\delta^{\prime}<\boldsymbol{\delta}_{1}^{2}$ (that is, there is a function sending $\alpha$ to a $\boldsymbol{\Sigma}_{1}\left(J_{\delta^{\prime}}(\mathbb{R})\right)$ definition of $\left.B_{\alpha}\right)$. This shows that $A^{\prime} \subseteq \mu^{\prime \omega}$ is Suslin, co-Suslin in $L(\mathbb{R})$, and thus determined. By the Coding Lemma, a winning strategy for $A^{\prime}$ lies in $J_{\delta}(\mathbb{R})$, a contradiction.

It is easy to see that I cannot win this game, for II just enumerates an honest set containing I's first move $\alpha$ (relative to $\phi_{n}^{s(\alpha)}$ ) and plays an appropriate $u$. A winning strategy for II gives $F$.

We fix this $F$ for the proof of the theorem below.

We shall deal below with Lipschitz continuous functions $\sigma: \mu^{\omega} \rightarrow \omega^{\omega}$ such that for $\alpha<\mu, \vec{\beta} \in s(\alpha)^{\omega}$ and $\vec{t}=(\alpha, \vec{\beta})$ enumerating an honest set $T, y=\sigma(\vec{t})$ codes a comeager set $A_{y} \subseteq T^{\omega}$ and a continuous function $f_{y}: A_{y} \rightarrow \omega^{\omega}$. By this we mean precisely that $\sigma$ is a strategy for II in a game where I plays $\alpha<\mu, \beta_{1}, \beta_{3}, \ldots<s(\alpha)$, II plays $\beta_{2}, \beta_{4}, \ldots<s(\alpha)$ and $y(0), y(1), \ldots$ If the $\alpha, \vec{\beta}$ enumerate an honest set $T$, then we require $y$ to code the comeager set $A_{y}$ and the continuous function $f_{y}$ as follows. $y$ codes dense open sets $D_{n} \subseteq T^{\omega}$, and $A_{y}=\bigcap_{n} D_{n}$. Also, $D_{n}=$ $\bigcup\left\{N_{s}: s \in T^{<\omega} \wedge \exists m y\left(\left\langle 0, n, m,\left\langle 2 a_{0}, \ldots, 2 a_{k}\right\rangle\right\rangle\right)=1 \wedge s=\left(\beta_{2 a_{0}}, \ldots, \beta_{2 a_{k}}\right)\right\}$. Here $N_{s}=\left\{\vec{t} \in T^{\omega}: \vec{t}\right.$ extends $\left.s\right\}$. Also, $y$ codes the continuous function $f_{y}$ : $A_{y} \rightarrow \omega^{\omega}$ by: $f_{y}\left(A_{y} \cap N_{\left(\beta_{2 a_{0}}, \ldots, \beta_{2 a_{k}}\right)}\right) \subseteq N_{r}$, for $r$ coding a sequence in $\omega<\omega$, iff $y\left(\left\langle 1,\left\langle 2 a_{0}, \ldots, 2 a_{k}\right\rangle, r\right\rangle\right)=1$, and for all basic neighborhoods $N_{s}$ in $D_{k}, \exists r$ coding a sequence of length $k$ such that $f_{y}\left(N_{s}\right) \subseteq N_{r}$. Note that only II's ordinal moves are used in the coding. Also, for fixed ordinal moves $\vec{t}$ by I and II, the set of $y$ coding a comeager set $A_{y}$ and a continuous function $f_{y}: A_{y} \rightarrow \omega^{\omega}$ is $\Pi_{2}^{0}$.

Any $\sigma \in \omega^{\omega}$ codes a relation $R_{\sigma} \subseteq \bigcup_{\alpha<\mu}\left(s(\alpha)^{<\omega} \times s(\alpha) \times \omega\right)$ via the Uniform Coding Lemma and $S_{s(\alpha)}$-pre-well-orderings of $P_{s(\alpha)}$ of length $s(\alpha)$. Note that the analysis of [St2] shows that these pre-well-orderings are obtained uniformly in $\alpha$. Thus, uniformly in $\alpha$, the relation $R_{\sigma}^{\prime}\left(\left\langle x_{0}, \ldots, x_{n}\right\rangle, y, k\right) \leftrightarrow\left(\left(\left|x_{0}\right|, \ldots,\left|x_{n}\right|\right),|y|, k\right) \in$ $R_{\sigma}$ is in $S_{s(\alpha)}$. That is, $R_{\sigma}\left\lceil s(\alpha)^{<\omega} \times s(\alpha) \times \omega\right.$ is $S_{s(\alpha)}$ in the codes, uniformly in $\alpha$. We usually just write $\sigma(\vec{s}, \gamma, k)$ in place of $R_{\sigma}(\vec{s}, \gamma, k)$, or $\sigma(\vec{s})=(\gamma, k)$ if $\sigma$ defines a strategy.

The next result is the main theorem of this section.

Theorem 5.10. If $k$ is proper, then it has the strong polarized partition property.

Proof. Let $\mu$ be the length of $k$. We define the function $\phi$ which together with the $\Gamma_{\alpha}$ satisfies Definition 5.2. Let $\sigma \in \omega^{\omega}, \alpha<\mu, \beta, \gamma \in\left[\sup _{\alpha^{\prime}} k\left(\alpha^{\prime}\right), k(\alpha)\right)$. We define $\phi(\sigma)(\beta, \gamma)$ to hold provided:

1. $\sigma\left\lceil s(\alpha)^{<\omega} \times s(\alpha) \times \omega\right.$ is a strategy for II. If $\vec{t}=(\alpha, \vec{\beta}) \in s(\alpha)^{\omega}$, let $\sigma(\vec{t})$ denote the real formed from the integer moves of $\sigma$ against $\vec{t}$.

2. $\forall^{*} T \in \mathcal{P}_{\omega_{1}}(s(\alpha)) \forall^{*} \vec{t}=(\alpha, \vec{\beta}) \in T^{\omega} \sigma(\vec{t})$ codes a comeager set $A_{\sigma(\vec{t})} \subseteq T^{\omega}$ and a continuous function $\sigma(\vec{t},-): A_{\sigma(\vec{t})} \rightarrow \omega^{\omega}$ such that $\forall^{*} \vec{t}_{1} \in T^{\omega}$, if $x=\sigma\left(\vec{t}, \vec{t}_{1}\right), u=F\left(\vec{t}_{1}\right)$, then $x \in R_{\alpha, \beta, \gamma}^{u}$.

To finish the proof of the theorem, it suffices to show properties (1)-(5) of Definition 5.2 for the coding $\phi$. Property (1) is trivial.

To verify (2), let $H: \lambda \rightarrow \lambda$ be a block function, where $\lambda=\sup _{\alpha<\mu} k(\alpha)$. Play the game where I plays $\alpha<\mu$, then I and II alternate playing out $\beta_{1}, \beta_{2}, \ldots<s(\alpha)$, and 
II makes additional integer moves $y(0), y(1), \ldots$, building up $y \in \omega^{\omega}$. Let $\vec{t}=(\alpha, \vec{\beta})$, and $T$ be the set they enumerate. Let $\vec{t}^{\prime}=\left(\left|G(\vec{t})_{0}\right|_{s(\alpha)},\left|G(\vec{t})_{1}\right|_{s(\alpha)}, \ldots,\right)$, and $T^{\prime}$ the set enumerated by $\vec{t}^{\prime}$ (thus $T=T^{\prime}$ if $T$ is honest). II wins provided $y$ codes a comeager set $A_{y} \subseteq\left(T^{\prime}\right)^{\omega}$ and a continuous function $f_{y}: A_{y} \rightarrow \omega^{\omega}$ such that for all $\vec{t}_{1} \in A_{y}$, if $u=F(\vec{t})$ and $w=f_{y}\left(\vec{t}_{1}\right)$, then $\phi_{u}(w)=H \uparrow\left[\sup _{\alpha^{\prime}<\alpha} k\left(\alpha^{\prime}\right), k(\alpha)\right) \times$ [ $\left.\sup k\left(\alpha^{\prime}\right), k(\alpha)\right)$. This game is determined by the usual reflection arguments (as $\alpha^{\prime}<\alpha$

before; see also $[\mathrm{KeW}])$ since the payoff depends only on $G(\vec{t})$. I cannot have a winning strategy $\sigma$, for if $\sigma$ 's first move is $\alpha$, II enumerates an honest subset of $s(\alpha)$ containing $\alpha$ closed under the strategy $\sigma$, and enumerates also a $y \in \omega^{\omega}$ as required for $T=T^{\prime}$. The fact that $y$ exists follows easily from the fact that any relation $R \subseteq \omega^{\omega} \times \omega^{\omega}$ can be uniformized on a comeager set by a continuous function. A winning strategy $\tau$ for II produces the desired code for $H$.

Property (3) follows easily from (4), since a set $R_{\alpha}$ which is a $k(\alpha)$ intersection of sets, each of which is a $k(\alpha)$ union of sets in $\boldsymbol{\Delta}_{\alpha}$, lies in $\boldsymbol{\Gamma}_{\alpha+1}$ since $\boldsymbol{\Delta}_{\alpha+1}$ is closed under $k(\alpha)$ unions and intersections from Lemma 5.6.

To verify (4), fix $\alpha<\mu, \beta, \gamma \in\left[\sup _{\alpha^{\prime}<\alpha} k\left(\alpha^{\prime}\right), k(\alpha)\right)$. Consider first the case where $\boldsymbol{\Gamma}_{\alpha}=\exists^{\omega^{\omega}} \boldsymbol{\Lambda}$ for some $\boldsymbol{\Lambda}$ closed under $\forall^{\omega^{\omega}}$ contained in $\boldsymbol{\Delta}_{\alpha}$. Thus, $\boldsymbol{\Lambda}$ is closed under finite unions and intersections. Fix a universal set $U \subseteq\left(\omega^{\omega}\right)^{3}$ for $\boldsymbol{\Lambda}$ subsets of $\left(\omega^{\omega}\right)^{2}$. By a game argument as above, there is a strategy $\tau: s(\alpha)^{\omega} \rightarrow s(\alpha)^{\omega} \times \omega$ such that for all $\vec{\beta} \in s(\alpha)^{\omega}$ with $\vec{t}=(\alpha, \vec{\beta})$ enumerating an honest set $T$ closed under $\tau, \tau(\vec{t})$ codes a comeager set $A_{\tau(\vec{t})} \subseteq T^{\omega}$ and a continuous function $\tau(\vec{t},-): A_{\tau(\vec{t})} \rightarrow \omega^{\omega}$ such that for $\vec{t}_{1} \in A_{\tau(\vec{t})}$, if $w_{1}=\tau\left(\vec{t}, \vec{t}_{1}\right)$, and $u=F\left(\vec{t}_{1}\right)$, we have $\forall x\left[R_{\beta, \gamma}^{u} \leftrightarrow \exists r U(w, x, r)\right]$. Suppose now $\sigma \in R_{\alpha, \beta, \gamma}$. By the game argument again, there is a strategy $\rho$ : $s(\alpha)^{\omega} \rightarrow s(\alpha)^{\omega} \times \omega$ such that for all $\vec{\beta} \in s(\alpha)^{\omega}$ with $\vec{t}=(\alpha, \vec{\beta})$ enumerating an honest set $T$ closed under $\rho, \rho(\vec{t})$ codes a comeager set $A_{\rho(\vec{t})} \subseteq T^{\omega}$ and a continuous function $\rho(\vec{t},-): A_{\rho(\vec{t})} \rightarrow \omega^{\omega}$ such that $\forall \vec{t}_{0} \in A_{\rho(\vec{t})}, \rho\left(\vec{t}, \vec{t}_{0}\right)$ codes a comeager set $A_{\rho\left(\vec{t}, \vec{t}_{0}\right)} \subseteq T^{\omega}$ and a continuous function $\rho\left(\vec{t}, \vec{t}_{0},-\right): A_{\rho\left(\vec{t}, \vec{t}_{0}\right)} \rightarrow \omega^{\omega}$ such that for all $\vec{t}_{1} \in A_{\rho\left(\vec{t}, \vec{t}_{0}\right)}$ we have: if $x=\sigma\left(\vec{t}_{0}, \vec{t}_{1}\right), w=\tau\left(\vec{t}_{0}, \vec{t}_{1}\right)$, then $U\left(w, x, \rho\left(\vec{t}, \vec{t}_{0}, \vec{t}_{1}\right)\right)$. Thus we have:

$$
\begin{aligned}
& \exists r \in \omega^{\omega}\left(r \text { codes a strategy } \rho: s(\alpha)^{\omega} \rightarrow s(\alpha)^{\omega} \times \omega\right) \\
& \text { such that } \forall T \in \mathcal{P}_{\omega_{1}}(s(\alpha)) \forall \vec{t} \text { enumerating } T[(T \\
& \text { closed under } \rho) \rightarrow\left(\rho(\vec{t}) \text { codes a comeager } A_{\rho(\vec{t})}\right. \\
\sigma \in R_{\alpha, \beta, \gamma} \Leftrightarrow & \text { and a continuous } \left.\rho(\vec{t},-): A_{\rho(\vec{t})} \rightarrow \omega^{\omega}\right) \wedge \forall \vec{t}_{0} \in \\
& A_{\rho(\vec{t})} \rho\left(\vec{t}, \overrightarrow{t_{0}}\right) \text { codes a comeager } A_{\rho\left(\vec{t}, \vec{t}_{0}\right)} \text { and a con- } \\
& \text { tinuous } \rho\left(\vec{t}, \vec{t}_{0},-\right): A_{\rho\left(\vec{t}, \vec{t}_{0}\right)} \rightarrow \omega^{\omega} \text { such that } \\
& \forall t_{1} \in A_{\rho\left(\vec{t}, \vec{t}_{0}\right)}\left[\text { if } x=\sigma\left(\vec{t}_{0}, \vec{t}_{1}\right), w=\tau\left(\vec{t}_{0}, \vec{t}_{1}\right),\right. \text { then } \\
& \left.\left.U\left(w, x, \rho\left(\vec{t}, \vec{t}_{0}, \vec{t}_{1}\right)\right)\right]\right] .
\end{aligned}
$$

This shows that $R_{\alpha, \beta, \gamma} \in \boldsymbol{\Gamma}_{\alpha}$. To see this, note that ( $r$ codes a strategy $\rho$ : $\left.s(\alpha)^{\omega} \rightarrow s(\alpha)^{\omega} \times \omega\right)$ is a $\forall^{\omega} S_{s(\alpha)}$ relation on $r$, using the Coding Lemma applied to the pointclass $S_{s(\alpha)}$ (there is a $S_{s(\alpha)}$ pre-well-ordering of length $s(\alpha)$ ). Likewise, to say $b$ codes a sequence $\vec{t}$ closed under $\rho$ is an $S_{s(\alpha)}$ relation, and also to say $\rho(\vec{t})=c$ defines an $S_{s(\alpha)}$ relation in $b, c$. Since $\forall^{\omega^{\omega}} S_{s(\alpha)} \subseteq \boldsymbol{\Lambda}$, the claim follows. A 
similar computation shows $\neg R_{\alpha, \beta, \gamma} \in \boldsymbol{\Gamma}_{\alpha}$ (use a strategy $\tau$ as above with $\tau\left(\vec{t}, \vec{t}_{1}\right.$ ) coding $\left.\left\{x: R_{\beta, \gamma}^{u}\right\}^{c}\right)$.

The proof when $\boldsymbol{\Delta}_{\alpha}$ is closed under real quantification is similar, so we just give a sketch. An easy game argument shows that if $\operatorname{cof}(\kappa)>s(\alpha)$ and $G: \mathcal{P}_{\omega_{1}}(s(\alpha)) \rightarrow \kappa$, then $\exists \delta<\kappa \forall^{*} T \in \mathcal{P}_{\omega_{1}}(s(\alpha)) G(\vec{t})<\delta$. Using this and the additivity of category it follows that $\exists \delta<k(\alpha)$ such that $\forall^{*} T \in \mathcal{P}_{\omega_{1}}(s(\alpha)) \forall^{*} \vec{\beta} \in T^{\omega}$ if $\vec{t}=(\alpha, \vec{\beta})$ and $u=F(\vec{t})$, then $R_{\beta, \gamma}^{u}$ has Wadge degree $\leq \delta$. Fix a universal set $U \subseteq \omega^{\omega} \times \omega^{\omega}$ for some non-selfdual pointclass of Wadge degree $\delta \leq o(U)<k(\alpha)$. There is a strategy $\tau: s(\alpha)^{\omega} \rightarrow s(\alpha)^{\omega} \times \omega$ such that if $\vec{t}=(\alpha, \vec{\beta})$ enumerates an honest set $T$ closed under $\tau$, then $\tau(\vec{t})$ codes a comeager set $A_{\tau(\vec{t})}$ and a continuous function $\tau(\vec{t},-): A_{\tau(\vec{t})} \rightarrow \omega^{\omega}$ such that $\forall \vec{t}_{1} \in A_{\tau(\vec{t})}$, if $u=F\left(\vec{t}_{1}\right)$ and $w=\tau\left(\vec{t}, \vec{t}_{1}\right)$, then $U_{w}=R_{\beta, \gamma}^{u}$. We may then compute directly:

$$
\sigma \in R_{\alpha, \beta, \gamma} \Leftrightarrow \begin{aligned}
& \forall^{*} T \in \mathcal{P}_{\omega_{1}}(s(\alpha)) \forall^{*} \vec{t}=(\alpha, \vec{\beta}) \in \\
& T^{\omega} \forall^{*} \vec{t}_{1} \in T^{\omega} U\left(\tau\left(\vec{t}, \vec{t}_{1}\right), \sigma\left(\vec{t}, \vec{t}_{1}\right)\right) .
\end{aligned}
$$

Since $\boldsymbol{\Delta}_{\alpha}$ is closed under quantifiers, this shows $R_{\alpha, \beta, \gamma} \in \boldsymbol{\Delta}_{\alpha}$.

Finally, we verify (5). Assume first again that $\boldsymbol{\Gamma}_{\alpha}=\exists^{\omega}{ }^{\omega} \boldsymbol{\Lambda}$ as above. Fix $\alpha<\mu$, $\beta \in\left[\sup _{\alpha^{\prime}<\alpha} k\left(\alpha^{\prime}\right), k(\alpha)\right)$, and suppose $D \subseteq R_{\alpha, \beta}$ is in $\exists^{\omega^{\omega}} \boldsymbol{\Delta}_{\alpha}$. Let now $\tau: s(\alpha)^{\omega} \rightarrow \omega$ be such that $\forall^{*} T \in \mathcal{P}_{\omega_{1}}(s(\alpha)) \forall^{*} \vec{t}=(\alpha, \vec{\beta}) \in T^{\omega} \tau(\vec{t})$ codes a comeager $A_{\tau(\vec{t})} \subseteq T^{\omega}$ and a continuous function $\tau(\vec{t},-): A_{\tau(\vec{t})} \rightarrow \omega^{\omega}$ such that $\forall \vec{t}_{1} \in A_{\tau(\vec{t})}$ if $w=\tau\left(\vec{t}, \vec{t}_{1}\right)$ and $u=F\left(\vec{t}_{1}\right)$, then $B_{w} \doteq\left\{\left(x_{1}, x_{2}\right): \exists r U\left(w,\left\langle x_{1}, x_{2}\right\rangle, r\right)\right\}$ along with $\boldsymbol{\Gamma}_{\alpha}, \phi_{u}$ witness the very reasonableness of $k(\alpha)$. Define a relation $\prec$ on $D$ by:

$$
\begin{aligned}
\sigma_{1} \prec \sigma_{2} \Leftrightarrow \quad & \forall^{*} T \in \mathcal{P}_{\omega_{1}}(s(\alpha)) \forall^{*} \vec{t}=(\alpha, \vec{\beta}) \in T^{\omega} \forall^{*} \vec{t}_{1} \in T^{\omega} \text { [if } \\
& x_{1}=\sigma_{1}\left(\vec{t}, \vec{t}_{1}\right), x_{2}=\sigma_{2}\left(\vec{t}, \vec{t}_{1}\right), w=\tau\left(\vec{t}, \vec{t}_{1}\right), \text { then } \\
& \left.\left.x_{1}, x_{2}\right) \in B_{w}\right] \\
& \exists r \in \omega^{\omega}\left(r \text { codes a strategy } \rho: s(\alpha)^{\omega} \rightarrow s(\alpha)^{\omega} \times \omega\right) \\
\Leftrightarrow \quad & \forall^{*} T \in \mathcal{P}_{\omega_{1}}(s(\alpha)) \forall^{*} \vec{t}=(\alpha, \vec{\beta}) \in T^{\omega} \forall^{*} \vec{t}_{0} \in T^{\omega} \\
& \forall^{*} \vec{t}_{1} \in T^{\omega}\left[\text { if } x_{1}=\sigma_{1}\left(\vec{t}_{0}, \vec{t}_{1}\right), x_{2}=\sigma\left(\vec{t}, \vec{t}_{1}\right), w=\right. \\
& \left.\tau\left(\vec{t}_{0}, \vec{t}_{1}\right), \text { then } U\left(w,\left\langle x_{1}, x_{2}\right\rangle, \rho\left(\vec{t}, \vec{t}_{0}, \vec{t}_{1}\right)\right)\right] .
\end{aligned}
$$

This shows $\prec \in \boldsymbol{\Gamma}_{\alpha}=\exists \exists^{\omega} \boldsymbol{\Delta}_{\alpha}$. Easily $\prec$ is a pre-well-ordering, and thus has length $\delta<k(\alpha)$. The regularity of $k(\alpha)$ then gives that $\sup \{\phi(\sigma)(\beta): \sigma \in D\}<$ $k(\alpha)$, verifying (5) in this case. The case where $\boldsymbol{\Delta}_{\alpha}$ is closed under quantifiers is easier, and is left to the reader.

This completes the proof of Theorem 5.10.

We note that the proof of Theorem 5.10 actually showed that the sequence $k$ is very reasonable.

Corollary 5.11. Let $\mu \leq \sup _{n \in \omega} \boldsymbol{\delta}_{n}^{1}$. Then any increasing, discontinuous sequence $\{k(\alpha)\}_{\alpha<\mu}$ of very reasonable cardinals has the strong polarized partition property.

Proof. Split the sequence into an initial part $\{k(\alpha)\}_{\alpha \leq \omega+1}$ and a terminal part $\{k(\alpha)\}_{\omega+1<\alpha<\mu}$. From Theorem 5.10, $\{k(\alpha)\}_{\omega+1<\alpha<\mu}$ is very reasonable, say witnessed by $\psi$. The proof of Proposition 5.7 shows that from $\psi$ and $\left\{\phi_{\alpha}\right\}_{\alpha \leq \omega+1}$ witnessing the very reasonableness of $k(\alpha)$ for $\alpha \leq \omega+1$ we may construct a $\phi$ witnessing the very reasonableness of the entire sequence $\{k(\alpha)\}_{\alpha<\mu}$. 
Using the same sort of argument, we may extend the corollary somewhat further.

Corollary 5.12. Let $\mu<$ the first inaccessible cardinal. Then any increasing, discontinuous $\mu$-sequence $k$ of very reasonable cardinals has the strong polarized partition property.

Proof. (sketch) Suppose not, and let $\mu \in O N$ be least such that there is an increasing, discontinuous sequence $k$ of very reasonable cardinals which is not very reasonable. The proof of Proposition 5.7 shows that $\mu$ is a limit ordinal of uncountable cofinality. Note that $\mu<\kappa^{\mathbb{R}}$, the Wadge ordinal of the class of inductive sets (the first non-selfdual pointclass closed under real quantification). Also, [St1] shows that for $\kappa<\kappa^{\mathbb{R}}, S_{s(\kappa)}$ (recall $S_{s(\kappa)}$ denotes the $s(\kappa)$-Suslin sets; here $s(\kappa)$ is the next Suslin cardinal after $\kappa$ ) is the next non-selfdual pointclass closed under $\exists \exists^{\omega}$ after $S(\kappa)$, that is, $\exists^{\omega^{\omega}} \breve{S}(\kappa)$. An easy induction then shows $\forall \alpha<\mu S_{s(\alpha)} \subseteq \boldsymbol{\Gamma}_{\alpha}$. Using Lemma 5.5, one has $\forall^{\omega} S_{s(\alpha)} \subseteq \boldsymbol{\Delta}\left(\boldsymbol{\Gamma}_{\alpha+1}\right)$.

Suppose first $\mu=\kappa^{+}$for some cardinal $\kappa$. Then, $\forall \alpha<\mu \forall^{\omega}{ }^{\omega} S_{s(\alpha)} \subseteq \boldsymbol{\Delta}\left(\boldsymbol{\Gamma}_{\kappa+1}\right)$. Thus, we split the sequence into the initial part $k \uparrow(\kappa+1)$ and a terminal part $k \uparrow(\mu \backslash \kappa)$. A coding function $\psi$ can be found for the terminal sequence from Theorem 5.10, and a coding function $\phi$ for the initial part by induction. The proof of Proposition 5.7 allows us to combine these to produce the desired coding function for the entire sequence.

We may assume now $\operatorname{cof}(\mu)=\lambda<\mu$. Fix an increasing, continuous, cofinal map $\pi: \lambda \rightarrow \mu$. By the splitting argument, we may assume $\forall^{\omega} S_{s(\lambda)} \subseteq \boldsymbol{\Delta}\left(\boldsymbol{\Gamma}_{0}\right)$. For $\delta<\lambda$, by the " $\delta^{t h " ~-b l o c k ~ w e ~ m e a n ~}\left[\sup _{\delta^{\prime}<\delta} k\left(\pi\left(\delta^{\prime}\right)\right), k(\pi(\delta))\right)$. By induction, for all $\delta<\lambda$ there is a very reasonable coding function for the $\delta^{t h}$-block (though we may not be able to choose them uniformly). The proof of Theorem 5.10, however, goes through for "blocks" of cardinals which are very reasonable (in Theorem 5.10 the blocks were singletons). This shows the entire sequence is very reasonable, a contradiction.

The first two paragraphs of the previous proof actually show the following.

Corollary 5.13. Let $\mu \leq \kappa^{\mathbb{R}}$, and $k$ an increasing, discontinuous $\mu$-sequence of very reasonable cardinals such that for all limit $\alpha<\mu, k(\alpha)>$ the least very reasonable cardinal greater than $\sup _{\alpha^{\prime}<\alpha} k\left(\alpha^{\prime}\right)$. Then $k$ has the strong polarized partition property.

Corollary 5.12 shows that any sequence of very reasonable cardinals of not too great a length has the strong polarized partition property. Corollary 5.13 allows greater length sequences, but imposes a mild "spreading out" condition on the sequence. The next result shows that we may obtain sequences of any length $<\Theta$ with the strong polarized partition property, provided we spread the $k(\alpha)$ sufficiently. The proof is easier than that of Theorem 5.10, and uses only the Coding Lemma.

Theorem 5.14. Let $\mu<\Theta$. Then there is an increasing, discontinuous $\mu-$ sequence $k$ of regular cardinals having the strong polarized partition property.

Proof. We construct a $\delta \geq \mu$ sequence $k$ of cardinals and corresponding pointclasses $\Gamma_{\alpha}$ satisfying: 
1. There is a pre-well-ordering $\prec$ of length $\delta$ such that $\forall \alpha<\delta \prec \uparrow \alpha+1 \in \boldsymbol{\Delta}_{\alpha}$. Here, $\prec \uparrow \alpha$ denotes the pre-well-ordering restricted to reals of rank $<\alpha$.

2. $\boldsymbol{\Gamma}_{\alpha}$ is closed under quantifiers and has the pre-well-ordering property uniformly in $\alpha$. That is, there is a function which assigns to $\alpha<\delta$ a $\boldsymbol{\Gamma}_{\alpha}$-universal set $A_{\alpha}$ and a $\boldsymbol{\Gamma}_{\alpha}$-pre-well-ordering $\psi_{\alpha}$ on $A_{\alpha}$. Also, $k(\alpha)$ is the supremum of the lengths of the $\boldsymbol{\Delta}_{\alpha}$ pre-well-orderings.

Granting this, we define the desired coding function $\phi$ as follows. Via the Uniform Coding Lemma and the pre-well-ordering $\prec$, every real $x$ codes a relation $R_{x} \subseteq$ $\operatorname{dom}(\prec) \times \omega^{\omega}$. Likewise, for all $\alpha<\delta$, every real $z$ codes a relation $R_{z}^{\alpha}$ with $\operatorname{dom}\left(A_{\alpha}\right)$ via the Uniform Coding Lemma and the pre-well-ordering $\psi_{\alpha}$. For $\alpha<\delta$ and $\beta, \gamma \in\left[\sup _{\alpha^{\prime}<\alpha} k\left(\alpha^{\prime}\right), k(\alpha)\right)$ we define

$$
\begin{aligned}
& \exists y, z, w_{1}, w_{2}\left[y \in \operatorname{dom}(\prec) \wedge|y|_{\prec}=\alpha \wedge R_{x}(y, z)\right. \\
& \wedge w_{1}, w_{2} \in A_{\alpha} \wedge\left|w_{1}\right|_{\psi_{\alpha}}=\beta \wedge\left|w_{2}\right|_{\psi_{\alpha}}=\gamma \\
& \phi(x)(\beta, \gamma) \Leftrightarrow \wedge R_{z}^{\alpha}\left(w_{1}, w_{2}\right) \wedge \forall y^{\prime}, z^{\prime}, w_{1}^{\prime}, w_{2}^{\prime}\left[\left|y^{\prime}\right|_{\prec}=|y|_{\prec} \wedge\right. \\
& R_{x}\left(y^{\prime}, z^{\prime}\right) \wedge\left|w_{1}^{\prime}\right|_{\psi_{\alpha}}=\left|w_{1}\right|_{\psi_{\alpha}} \wedge R_{z}^{\alpha}\left(w_{1}^{\prime}, w_{2}^{\prime}\right) \rightarrow \\
&\left.\left.\left(w_{2}^{\prime} \in A_{\alpha} \wedge\left|w_{2}^{\prime}\right|_{\psi_{\alpha}}=\left|w_{2}\right|_{\psi_{\alpha}}\right)\right]\right] .
\end{aligned}
$$

It is straightforward to check properties (1)-(5) of Definition 5.2 for the coding $\phi$. To check (4), for example, note that for $\alpha, \beta, \gamma$ as above, $R_{\alpha, \beta, \gamma}=\{x: \phi(x)(\beta, \gamma)\} \in$ $\boldsymbol{\Delta}_{\alpha}$, as $\boldsymbol{\Delta}_{\alpha}$ is closed under quantifiers. (5) follows easily from the fact that every $\boldsymbol{\Delta}_{\alpha}$ subset of $A_{\alpha}$ is bounded below $k(\alpha)$ with respect to $\psi_{\alpha}$.

The $k(\alpha), \boldsymbol{\Gamma}_{\alpha}$ may be constructed in several ways. For example, let $\boldsymbol{\Gamma}=\boldsymbol{\Sigma}_{1}^{2}(\mu)$ $=\boldsymbol{\Sigma}_{1}(L(\mathbb{R}) ; \mu) \stackrel{\text { def }}{=}$ the sets $\boldsymbol{\Sigma}_{1}$ definable in $L(\mathbb{R})$ from parameters $\mu$ and reals. Then $\boldsymbol{\Gamma}$ is closed under countable unions, intersections, $\exists^{\omega^{\omega}}, \forall^{\omega}{ }^{\omega}$. The pointclass $\Sigma_{1}^{2}(\mu)$ resembles $\boldsymbol{\Sigma}_{1}^{2}$ (except it doesn't have the scale property). Let $\delta$ be the least ordinal such that $L_{\delta}(\mathbb{R}) \prec_{1}^{\mu} L(\mathbb{R})$, that is, elementary for $\boldsymbol{\Sigma}_{1}$ formulas with parameter $\mu$ (and reals). Then $\boldsymbol{\Gamma}$ has Wadge degree $\delta$, and $\boldsymbol{\Delta}=\bigcup_{\alpha<\delta} L_{\alpha}(\mathbb{R}) \cap \omega^{\omega}$ (cf. Lemma 1.12 of [St2]). Define $k(\alpha), \boldsymbol{\Gamma}_{\alpha}$ for $\alpha<\delta$ by induction: let $k(\alpha)$ be the least ordinal $>\sup _{\alpha^{\prime}<\alpha} k\left(\alpha^{\prime}\right)$ such that $\boldsymbol{\Gamma}_{\alpha} \stackrel{\text { def }}{=} \boldsymbol{\Sigma}_{1}\left(L_{k(\alpha)}(\mathbb{R}) ; \mu\right)$ is closed under countable unions, intersections, $\exists{ }^{\omega}, \forall \omega^{\omega}$, and is not equal to $\boldsymbol{\Sigma}_{1}\left(L_{\beta}(\mathbb{R}) ; \mu\right)$ for any $\beta<k(\alpha)$. The regularity of $\delta$ and a reflection argument show that $k(\alpha)$ is well-defined. For $\prec$, we may take the natural pre-well-ordering on reals $x$ viewed as coding $\Sigma_{1}(\bar{x}, \mu)$ statements $\theta_{x}$ (where $\left.\bar{x}(n)=x(n+1)\right)$. Namely, $x \prec y$ iff $\exists \alpha L_{\alpha}(\mathbb{R}) \models \theta_{x} \wedge \neg \theta_{y}$. Likewise we define $\psi_{\alpha}$ (so $\psi_{\alpha}$ is an initial segment of $\prec$ ).

In view of Corollary 5.12 and Theorem 5.14, the following conjecture seems plausible.

Every increasing, discontinuous $\mu$-sequence $k$ of (very) reasonable cardinals of length $\mu<\Theta$ has the strong polarized partition property.

A proof of this conjecture may depend on further extensions of the detailed $L(\mathbb{R})$ analysis.

Finally, we present a result which extends Theorem 5.10 to partitions into more than 2 pieces.

Proposition 5.15. Suppose $k$ is a proper $\mu$-sequence. Then $k$ has the strong polarized partition property for partitions into $<k(0)$ many pieces.

Proof. Fix $k$ and the associated pointclasses $\boldsymbol{\Gamma}_{\alpha}$, and fix a partition $\mathcal{P}$ of the block functions into $\delta<k(0)$. For all $\beta<\delta$, $\mathcal{P}$ induces a partition $\mathcal{P}_{\beta}$ defined by: 
$\mathcal{P}_{\beta}(F)=1$ iff $\mathcal{P}(F)=\beta$. Towards a contradiction, we may assume each $\mathcal{P}_{\beta}$ is homogeneous for the 0 side.

From Lemmas 5.5, 5.6 it follows that for all $\alpha<\mu$ there is a pre-well-ordering of length $k(\alpha)$ such that every $\boldsymbol{\Delta}_{\alpha}$ subset of the field is bounded in the norm (use $\operatorname{PWO}\left(\check{\boldsymbol{\Gamma}}_{\alpha}\right)$ and the closure of $\boldsymbol{\Gamma}_{\alpha}$ under $\wedge$ which allows the usual boundedness argument to be carried out). By the usual game argument, there is a strategy $F$ such that $\forall \alpha<\mu \forall t=(\alpha, \vec{\beta}) \in s(\alpha)^{\omega}$ enumerating an honest set closed under $F$, $u=F(\vec{t})$ codes a set $B_{u}$ and a pre-well-ordering $\prec_{u}$ of $B_{u}$ with the boundedness property for $\boldsymbol{\Delta}_{\alpha}$ sets. Again, the exact manner in which $u$ codes these objects is not important, say by continuous pre-images of a fixed set of high Wadge degree.

For $\beta<\delta$, we say a real $\sigma$ is $\beta$-good if $\sigma$ codes a strategy (also denoted by $\sigma$ ) such that:

1. $\forall \alpha<\mu \forall \vec{t}=(\alpha, \vec{\beta}) \in s(\alpha)^{\omega}$ enumerating an honest set closed under $F$ and $\sigma$, $\sigma(\vec{t})$ codes a comeager set $A_{\sigma(\vec{t})}$ and a continuous function $\sigma(\vec{t},-): A_{\sigma(\vec{t})} \rightarrow \omega^{\omega}$ such that $\forall \vec{t}_{1} \in A_{\sigma(\vec{t})}$ if $w=\sigma\left(\vec{t}, \vec{t}_{1}\right), u=F\left(\vec{t}_{1}\right)$, then $w$ codes a strategy on $B_{u}$, that is, $\forall x \in B_{u} w(x) \in B_{u}$ (where we view $w$ as a strategy in an integer game).

2. For $\alpha<\mu$, define $G_{\sigma}^{\alpha}: k(\alpha) \rightarrow k(\alpha)$ as follows. If $\gamma<k(\alpha)$, let $G_{\sigma}^{\alpha}(\gamma)$ be the least ordinal $\eta$ such that $\forall^{*} T \in \mathcal{P}_{\omega_{1}}(s(\alpha)) \forall^{*} \vec{t}=(\alpha, \vec{\beta}) \in T^{\omega} \forall^{*} \vec{t}_{1} \in T^{\omega}$ if $w=\sigma\left(\vec{t}, \vec{t}_{1}\right), u=F\left(\vec{t}_{1}\right)$, then $\sup \left\{|w(x)|_{\prec_{u}}: x \in B_{u} \wedge|x|_{\prec_{u}} \leq \gamma\right\}<\eta$. It is not difficult to check that $G_{\sigma}^{\alpha}(\gamma)<k(\alpha)$. Let $C_{\sigma}^{\alpha} \subseteq\left[\sup _{\alpha^{\prime}<\alpha} k\left(\alpha^{\prime}\right), k(\alpha)\right)$ be the set of closure points of $G_{\sigma}^{\alpha}$. We then require that the sets $C_{\sigma}^{\alpha}$ are block homogeneous for $\mathcal{P}_{\beta}$.

Note that if $C \subseteq k(\alpha)$ is c.u.b., and $\prec_{u}$ is a pre-well-ordering of some set $B_{u}$ with the $\boldsymbol{\Delta}_{\alpha}$ boundedness property, then $\exists w \in \omega^{\omega} C_{w}^{\alpha} \subseteq C$, where $C_{w}^{\alpha}$ is the set of closure points of $G_{w}^{\alpha}$, and $G_{w}^{\alpha}(\gamma)=\sup \left\{|w(x)|_{\prec_{u}}: x \in B_{u} \wedge|x|_{\prec_{u}} \leq \gamma\right\}$. This follows by a simple Solovay game argument. The usual game argument (as in Theorem 5.10) then shows that $\forall \beta<\delta \exists \sigma \in \omega^{\omega} \sigma$ is $\beta$-good.

Fix a $\boldsymbol{\Delta}_{0}$ pre-well-ordering $\prec_{0}$ of length $\delta$. The Coding Lemma gives an $\exists^{\omega^{\omega}} \boldsymbol{\Delta}_{0}$ relation $R \subseteq \operatorname{dom}\left(\prec_{0}\right) \times \omega^{\omega}$ such that $\forall a \in \operatorname{dom}\left(\prec_{0}\right) \exists \sigma R(a, \sigma)$ and $\forall a \forall \sigma[R(a, \sigma) \wedge$ $|a|_{\prec_{0}}=\beta \rightarrow \sigma$ is $\beta$-good].

Define now a block function $G$ on $\sup _{\alpha<\mu} k(\alpha)$ as follows. For $\alpha<\mu$ and $\gamma \in$ $\left[\sup k\left(\alpha^{\prime}\right), k(\alpha)\right)$, define $G(\gamma)=\sup \left\{G_{\sigma}^{\alpha}(\gamma): \exists a \in \operatorname{dom}\left(\prec_{0}\right) R(a, \sigma)\right\}$. If $G$ really is a block function (i.e., $G(\gamma)<k(\alpha)$ ), then if we let $C$ be the set of closure points of $G, C$ is block homogeneous for all of the partitions $\mathcal{P}$ simultaneously, a contradiction.

So, fix $\alpha<\mu, \gamma \in\left[\sup _{\alpha^{\prime}<\alpha} k\left(\alpha^{\prime}\right), k(\alpha)\right)$. We must show that $G(\gamma)<k(\alpha)$. Suppose $T \in \mathcal{P}_{\omega_{1}}(s(\alpha))$ is an honest set containing $\alpha$ and closed under $F, \vec{t}=(\alpha, \vec{\beta})$ enumerates $T$, and $\vec{t}_{1} \in T^{\omega}$. Define $\sigma \in D_{\vec{t}, \vec{t}_{1}} \leftrightarrow \exists a R(a, \sigma) \wedge(T$ is closed un$\operatorname{der} \sigma) \wedge\left(\vec{t}_{1} \in A_{\sigma(\vec{t})}\right)$. A straightforward computation shows $D_{\vec{t}, \vec{t}_{1}} \in \exists^{\omega^{\omega}} \boldsymbol{\Delta}_{\alpha}$. By boundedness and the definition of $R$ it follows that $\theta_{\vec{t}, \vec{t}_{1}}<k(\alpha)$, where $\theta_{\vec{t}, \vec{t}_{1}}=$ $\sup \left\{|w(x)|_{\prec u}: \exists \sigma \in D_{\vec{t}, \vec{t}_{1}} w=\sigma\left(\vec{t}, \vec{t}_{1}\right) \wedge|x|_{\prec_{u}}=\gamma\right\}$, where $u=F\left(\vec{t}_{1}\right)$. By the additivity of category, $\forall^{*} T \in \mathcal{P}_{\omega_{1}}(s(\alpha)) \exists \theta<k(\alpha) \forall^{*} \vec{t}=(\alpha, \vec{\beta}) \in T^{\omega} \forall^{*} \vec{t}_{1} \in T^{\omega} \theta_{\vec{t}, \vec{t}_{1}}<\theta$. The game argument then shows $\exists \theta<k(\alpha) \forall^{*} T \in \mathcal{P}_{\omega_{1}}(s(\alpha)) \forall^{*} \vec{t}=(\alpha, \vec{\beta}) \in T^{\omega}$ 
$\forall^{*} \vec{t}_{1} \in T^{\omega} \theta_{\vec{t}, \vec{t}_{1}}<\theta$. However, if $\sigma \in \operatorname{ran}(R)$, then $\forall^{*} T \forall^{*} \vec{t} \forall^{*} \vec{t}_{1} \sigma \in D_{\vec{t}, \vec{t}_{1}}$. Hence, $G_{\sigma}^{\alpha}(\gamma) \leq \theta$ for all $\sigma \in \operatorname{ran}(R)$, and so $G(\gamma) \leq \theta$.

\section{Polarized Magidor-Like Forcing}

With the product of a number of Magidor-like partial orderings, we can change cofinalities of many cardinals simultaneously. Because of considerations of choice, we will restrict our attention to products that are at most countable. For this section, let $k$ be a countable sequence of cardinals with $\gamma$ an uncountable cardinal below $k(0)$. Let

$$
\mathcal{P}_{\gamma, k}=\left\{p \in \prod_{\kappa \in k} \mathcal{P}_{\gamma, \kappa}: \exists \delta<\gamma \forall \kappa \in k \text { either } p_{0}(\kappa)=\emptyset \text { or } p_{0}(\kappa) \in[\kappa]^{\delta}\right\} .
$$

If $p \in \mathcal{P}_{\gamma, k}$, let $p_{i}=\prod_{\kappa \in k} p(\kappa)_{i}$, for $i=0,1$. Extend the definition of $\mathbb{H}$ to $[k]^{<\gamma}$ in the natural way.

Many of the facts in section 3 have analogues here.

Proposition 6.1. If $(k) \rightarrow(k)^{<\gamma}, \phi$ is a formula in the forcing language with respect to $\mathcal{P}_{\gamma, k}$ and $p \in \mathcal{P}_{\gamma, k}$, then either $p \| \sim \phi$ or $p \| \sim \neg \phi$.

Proof. The proof is close to that of Fact 3.2 (1.1 of [H2]). Define $f:\left[p_{1}\right]^{<\gamma} \rightarrow 3$ by

$$
f(W)= \begin{cases}0 & \text { if } p_{0} \frown\left({ }_{\omega} W\right) \| \sim \phi, \\ 1 & \text { if } p_{0} \frown\left({ }_{\omega} W\right) \mid \sim \neg \phi, \\ 2 & \text { otherwise. }\end{cases}
$$

Let $C$ be homogeneous for $f$. We claim that $q=\left\langle p_{0}, C\right\rangle$ decides $\phi$. To see this, suppose that $r, r^{\prime} \Vdash q$ decide $\phi$ differently. We may assume that these are of uniform length, i.e., $r_{0}, r_{0}^{\prime} \in[k]^{\beta}$, for some $\beta<\gamma$. Using $\mathrm{AC}_{\omega}$, choose $t \in[C]^{<\gamma}$ such that $r_{0} \backslash p_{0}={ }_{\omega} t$, and choose $t^{\prime}$ similarly for $r^{\prime}$. Then $f(t) \neq f\left(t^{\prime}\right)$, contradicting the homogeneity of $C$. Finally, if $q \Vdash \phi$, then $p \| \sim \phi$ and if $q \Vdash \neg \phi$, then $p \| \sim \neg \phi$.

Proposition 6.2. If $(k) \rightarrow(k)^{<\gamma}$, then $\mathbf{V}^{\mathcal{P}_{\gamma, k}}$ and $\mathbf{V}$ have the same bounded subsets of $k(0)$.

Proof. This is a consequence of Proposition 6.1 together with Fact 4.7. See, for example, the proof of 1.3 in [H2].

We have two preservation theorems involving splitting a polarized sequence. Let $k_{0}, k_{1}$ be consecutive halves of $k$, that is, $k=k_{0} \frown k_{1}$.

Proposition 6.3. If $(k) \rightarrow(k)^{k}$, then $\mathbf{V}^{\mathcal{P}_{\gamma, k_{1}}} \vDash\left(k_{0}\right) \rightarrow\left(k_{0}\right)^{k_{0}}$.

Proof. Of course, $\left(k_{0}\right) \rightarrow\left(k_{0}\right)^{k_{0}}$ is true in $\mathbf{V}$ by Fact 4 .4. In $\mathbf{V}^{\mathcal{P}_{\gamma, k_{1}}}$, however, there may be new partitions. Note that since $\mathcal{P}_{\gamma, k_{1}}$ adds no new bounded subsets of $k_{1}(0)$, the set $\left[k_{0}\right]^{k_{0}}$ is the same in both $\mathbf{V}$ and $\mathbf{V}^{\mathcal{P}_{\gamma, k_{1}}}$, that is, there may be new partitions, but the domain remains the same. Suppose that $p \Vdash$ " $\dot{f}:\left[k_{o}\right]^{k_{0}} \rightarrow 2$ ".

With the methods used in the proof of Proposition 4.3, we can prove from $(k) \rightarrow$ $(k)^{k}$ that given any partition $g$ of $\left[k_{0}\right]^{k_{0}} \times\left[k_{1}\right]^{<\gamma}$, we can find a set $C \in[k]^{k}$ such that for each $\beta<\gamma, g$ is constant on $\left[k_{0}\right]^{k_{0}} \times\left[k_{1}\right]^{\beta}$. We could write this technical fact as:

$$
\left(\begin{array}{l}
k_{0} \\
k_{1}
\end{array}\right) \rightarrow\left(\begin{array}{c}
k_{0} \\
k_{1}
\end{array}\right)^{k_{0},<\gamma}
$$


Define a partition $g$ on this set into 3 as follows. If $W \in\left[k_{0}\right]^{k_{0}} \times\left[k_{1}\right]^{\beta}$,

$$
g(W)=i \text { if }\left[p_{0} \frown \omega\left(W \uparrow k_{1}\right)\right] \mid \downarrow * \dot{f}\left(W \uparrow k_{0}\right)=i, "
$$

and $g(W)=2$, otherwise.

Let $C$ be homogeneous for $g$. We claim that $q=\left\langle p_{0}, C\right\rangle$ forces that $\left[C\left\lceil k_{0}\right]^{k_{0}}\right.$ is homogeneous for $f$. If not, suppose $r, r^{\prime} \Vdash q$ and $r \Vdash " \dot{f}(x)=0 "$ and $r^{\prime} \Vdash$ " $\dot{f}\left(x^{\prime}\right)=1, " x, x^{\prime} \in\left[C \nmid k_{0}\right]^{k_{0}}$. We may assume as before that the sets $r(\kappa)_{0} \backslash p(\kappa)_{0}$ and $r^{\prime}(\kappa)_{0} \backslash p(\kappa)_{0}$ all have the same length and choose $t, t^{\prime} \in\left[C\left\lceil k_{1}\right]^{\beta}\right.$ so that $r_{0} \backslash p_{0}={ }_{\omega} t$ and $r_{0}^{\prime} \backslash p_{0}={ }_{\omega} t^{\prime}$. We can then put $x$ and $t$ together to form $W$ with $g(W)=0$. Similarly, with $x^{\prime}$ and $t^{\prime}$ we can form $W^{\prime}$ such that $g\left(W^{\prime}\right)=1$, a contradiction.

Forcing with the lower half of $k$ is more disruptive to the upper half than viceversa. Still, we can prove the following.

Proposition 6.4. If $(k) \rightarrow(k)^{<\omega_{1}}$, then $\mathbf{V}^{\mathcal{P}_{\omega_{1}, k_{0}}} \vDash\left(k_{1}\right) \rightarrow\left(k_{1}\right)^{<\omega_{1}}$.

Proof. The proof is quite similar to the proof of Proposition 6.3, reversing the roles of $k_{0}$ and $k_{1}$, and replacing the technical fact with:

$$
\left(\begin{array}{c}
k_{0} \\
k_{1}
\end{array}\right) \rightarrow\left(\begin{array}{c}
k_{0} \\
k_{1}
\end{array}\right)^{<\omega_{1},<\omega_{1}}
$$

by which we mean that for all partitions $f: \bigcup_{\alpha, \beta<\omega_{1}}\left[k_{0}\right]^{\alpha} \times\left[k_{1}\right]^{\beta} \rightarrow 2$, there is $X \in\left[k_{0}\right]^{k_{0}}, Y \in\left[k_{1}\right]^{k_{1}}$, with $f$ constant on $\bigcup_{\alpha, \beta<\omega_{1}}[X]^{\alpha} \times[Y]^{\beta}$ for all $\alpha, \beta<\omega_{1}$. Any such $f$ can easily be coded as a partition of $[k]^{<\omega_{1}}$, and so this relation follows from the hypothesis.

The restriction in the statement of the theorem to exponent " $<\omega_{1}$ " is needed because while $\left(\left[k_{1}\right]^{k_{1}}\right)^{\mathbf{V}} \neq\left(\left[k_{1}\right]^{k_{1}}\right)^{\mathbf{V}^{\mathcal{P}_{\gamma}, k_{0}}},\left(\left[k_{1}\right]^{<\omega_{1}}\right)^{\mathbf{V}}$ does equal $\left(\left[k_{1}\right]^{<\omega_{1}}\right)^{\mathbf{V}^{\mathcal{P}_{\gamma}, k_{0}}}$, since $\mathcal{P}_{\gamma, k_{0}}$ is countably closed and we are forcing over a model of DC.

Now if $p \Vdash$ " $\dot{f}:\left[k_{o}\right]^{<\omega_{1}} \rightarrow 2$ " we can define a partition $g$ of $\bigcup_{\alpha, \beta<\omega_{1}}\left[k_{0}\right]^{\alpha} \times\left[k_{1}\right]^{\beta}$ into 3 as follows. If $W \in\left[k_{0}\right]^{\alpha} \times\left[k_{1}\right]^{\beta}$,

$$
g(W)=i \text { if }\left[p_{0} \frown{ }_{\omega}\left(W \uparrow k_{0}\right)\right] \mid \sim " \dot{f}\left(W\left\lceil k_{1}\right)=i, "\right.
$$

and $g(W)=2$, otherwise.

Let $C$ be homogeneous for $g$. In the manner of Proposition 6.3, $q=\left\langle p_{0}, C\left\lceil k_{0}\right\rangle\right.$ forces that $\left[C\left\lceil k_{1}\right]^{k_{1}}\right.$ is homogeneous for $f$.

Using the methods of the previous two propositions, we can prove:

Proposition 6.5. If $(k) \rightarrow(k)^{<\gamma}$, then $\mathbf{V}^{\mathcal{P}_{\gamma, k_{1}}} \vDash\left(k_{0}\right) \rightarrow\left(k_{0}\right)^{<\gamma}$.

Simple factoring shows that finite products achieve exactly the cofinality changes intended. For infinite products, the picture is not as nice. We can, however, deal with countable products.

Proposition 6.6. Let $k$ be a countable sequence satisfying $(k) \rightarrow(k)^{<\gamma}, \gamma<k(0)$, and suppose that there is no non-principal ultrafilter on $\omega$. Then for all cardinals $\delta$ of $\mathbf{V}, \operatorname{cof}^{\mathbf{V}^{\mathcal{P}_{\gamma, k}}}(\delta)=\operatorname{cof}^{\mathbf{V}}(\delta)$ if $\operatorname{cof}^{\mathbf{V}}(\delta) \notin k$ and $\operatorname{cof}^{\mathbf{V}^{\mathcal{P}_{\gamma, k}}}(\delta)=\gamma$ otherwise.

Proof. By Proposition 6.2, this is certainly true for cardinals $\delta$ with $\operatorname{cof}(\delta) \leq k(0)$ (if the cofinality of $\delta$ is changed, then so is the cofinality of $\operatorname{cof}(\delta)$ ). We will next 
deal with the case: $\operatorname{cof}(\delta)>\bigcup k$ and show at the end how to handle cardinals $k(0)<\operatorname{cof}(\delta)<\bigcup k$.

Suppose that $p \Vdash " \dot{F}: \lambda \rightarrow \delta$ is unbounded", $\lambda<\operatorname{cof}^{\mathbf{V}}(\delta)$. For $s \in\left[p_{1}\right]^{<\gamma}$, let $n_{s}=\left\{\beta<\delta: \exists \alpha p_{0} \frown{ }_{\omega} s \| \sim \dot{F}(\alpha)=\beta\right\}$. Note that $\left\|n_{s}\right\|<\operatorname{cof}^{\mathrm{V}}(\delta)$ since no sequence can insinuate different values for a particular $\dot{F}(\alpha)$. Since $n_{s}$ is defined in $\mathbf{V}$ then, $\bigcup n_{s}<\delta$.

Claim. There is a $q \in\left[p_{1}\right]^{k}$ such that for any $\eta<\gamma, \bigcup_{s \in[q]^{\eta}} \bigcup n_{s}<\delta$. This will give us a contradiction, since then $\left\langle p_{0}, q\right\rangle$ will force that $\dot{F}$ is bounded.

Proof of Claim. We begin by defining $f:\left[p_{1}\right]^{<\gamma} \rightarrow 3$ by:

$$
f(s \frown t)= \begin{cases}0 & \text { if } \bigcup n_{s}=\bigcup n_{t} \\ 1 & \text { if } \bigcup n_{s}<\bigcup n_{t} \\ 2 & \text { if } \bigcup n_{s}>\bigcup n_{t},\end{cases}
$$

where $s \frown t \in[k]^{\eta+\eta}, \eta<\gamma$. Let $q_{1} \in\left[p_{1}\right]^{k}$ be homogeneous for $f$. Clearly, the range of $f$ on $[q]^{\eta+\eta}$ cannot be $\{2\}$. If the range is $\{0\}$ for a given $\eta$, then we have as promised that $\bigcup_{s \in\left[q_{1}\right]^{\eta}} \bigcup n_{s}<\delta$.

Suppose for some fixed $\eta f\left\lceil\left[q_{1}\right]^{\eta+\eta}=\{1\}\right.$. Let $\left\{\nu_{n}\right\}_{n<\omega}$ be an enumeration of $k$. For any $R \subseteq \omega$, define the partition $f_{R}^{\eta}:[k]^{\eta+\eta} \rightarrow 3$ by:

$$
f_{R}^{\eta}(s \frown t)= \begin{cases}0 & \text { if } \bigcup n_{s}=\bigcup n_{(s, t)_{R}} \\ 1 & \text { if } \bigcup n_{s}<\bigcup n_{(s, t)_{R}} \\ 2 & \text { if } \bigcup n_{s}>\bigcup n_{(s, t)_{R}},\end{cases}
$$

where $(s, t)_{R} \in[k]^{\eta}$ is defined:

$$
(s, t)_{R}\left(\nu_{n}\right)= \begin{cases}s\left(\nu_{n}\right) & \text { if } n \notin R \\ t\left(\nu_{n}\right) & \text { if } n \in R\end{cases}
$$

We don't have the power to find a set homogeneous for all of these partitions at once $\left[(k) \rightarrow(k)_{2 \omega}^{<\gamma}\right.$ is sufficient to produce a set homogeneous for $\omega$-many at once, but there are $2^{\omega}$-many partitions and $(k) \rightarrow(k)_{2^{2}}^{<\gamma}$ is generally false] but note two important facts. First, the range of $f_{R}^{\eta}$ on any homogeneous set cannot be $\{2\}$, or we could easily construct an infinite descending chain of ordinals. Second, if $q^{\prime}, q^{\prime \prime}$ are both homogeneous for a certain $f_{R}^{\eta}$, then the ranges of $f_{R}^{\eta}$ on the two are the same. This is because the partition is defined in terms of "pre-sub-omega" $(\omega)$, through the definition of $n_{s}$, and it is easy to find $s^{\prime} \in\left[q^{\prime}\right]^{\gamma}, s^{\prime \prime} \in\left[q^{\prime \prime}\right]^{\gamma}$ such that ${ }_{\omega} s^{\prime}={ }_{\omega} s^{\prime \prime}$.

For this reason we can unambiguously define $m(R)$ for $R \subseteq \omega$ as $i$ iff the range of $f_{R}^{\eta}$ on any homogeneous set is $\{i\} . m$ is like a measure. We can prove, for example, that if $R \subseteq W, m(W)=1$, then at least one of $m(R), m(W \backslash R)$ is 1 . To see this, choose $s \frown t \frown u$, an $\eta \cdot 3$-sequence from any set homogeneous for $f_{W}^{\eta}, f_{R}^{\eta}$, and $f_{W \backslash R}^{\eta}$. Then $f_{W}^{\eta}(s, t)=1$ implies $\bigcup n_{s}<\bigcup n_{(s, t)_{W}}$. But $f_{R}^{\eta}(s, t)=0$ implies $\bigcup n_{s}=\bigcup n_{(s, t)_{R}}$ and $f_{W \backslash R}^{\eta}\left((s, t)_{R},(t, u)_{R}\right)=0$ implies $\bigcup n_{(s, t)_{R}}=\bigcup n_{(s, t)_{W}}$. Similarly, if $R \subseteq W, m(W)=0$, then both $m(R), m(W \backslash R)$ are 0. This shows that $R \subseteq W \Rightarrow m(R) \leq m(W)$. Note that $m(\omega)=1$.

We claim next that there cannot be an infinite collection of mutually disjoint sets $\left\{R_{n}\right\}_{n<\omega}$ all with measure one, i.e., $m\left(R_{n}\right)=1$. This is because we could find $x$ homogeneous simultaneously for all the $f_{R_{n}}^{\eta}$, and then for $s \frown t \in[x]^{\eta+\eta}$ we would have

$$
\bigcup n_{(s, t)_{\omega}}>\bigcup n_{(s, t)_{\omega \backslash R_{0}}}>\bigcup n_{(s, t)_{\omega \backslash\left(R_{0} \cup R_{1}\right)}}>\ldots
$$


(If $s, t, u$ are as before, apply $f_{R_{0}}^{\eta}$ to $\left((s, t)_{\omega \backslash R_{0}},(t, u)_{\omega \backslash R_{0}}\right)$ for the first inequality, then apply $f_{R_{1}}^{\eta}$ to $\left((s, t)_{\omega \backslash\left(R_{0} \cup R_{1}\right)},(t, u)_{\omega \backslash\left(R_{0} \cup R_{1}\right)}\right)$ for the second inequality, and so on.) Let $Q^{\eta}=\{n: m(\{n\})=1\}$. From the discussion above, $Q^{\eta}$ must be finite.

Now we claim that $m\left(\omega \backslash Q^{\eta}\right)=0$. If not, we would have that for some infinite subset $R$ of $\omega \backslash Q^{\eta}, m(R)=1$ and $W \subseteq R$ implies that exactly one of the sets $W$, $R \backslash W$ is measure one (otherwise we could construct an infinite disjoint collection of measure one sets). In effect, this gives us a non-principal ultrafilter on $R$ (additivity follows from this and the properties proved above), contradicting a hypothesis of the proposition.

Let $x \in\left[q_{1}\right]^{k}$ be homogeneous for $f_{\omega \backslash Q^{\eta}}$. Take

$$
\zeta=\bigcup\left\{\bigcup n_{s}:\left[\begin{array}{l}
\text { if } n \in Q^{\eta}, \text { then } s\left(\nu_{n}\right) \text { is an interval of } x\left(\nu_{n}\right) \\
\text { if } n \notin Q^{\eta}, \text { then } s\left(\nu_{n}\right)=x\left(\nu_{n}\right)\lceil\eta
\end{array}\right]\right\} .
$$

Since $Q^{\eta}$ is finite, there are no more than $\nu_{n}$-many $s$ in the definition of $\zeta$, where $\nu_{n} \in k$ is greatest with $n \in Q^{\eta}$. Since $\operatorname{cof}(\delta)>\nu_{n}$, we must have $\zeta<\delta$. This then gives us $\bigcup_{s \in[x]^{\eta}} \cup n_{s}<\delta$ as follows: Suppose $s \in[x]^{\eta}$ and for some $y\left\langle p_{0} \frown{ }_{\omega} s, y\right\rangle \Vdash$ " $\dot{F}(\alpha)=\beta$ ". Take $t \in[x]^{\eta}, t>s$, such that $t\left(\nu_{n}\right)$ is an interval of $x\left(\nu_{n}\right)$ for all $n \in Q^{\eta}$. Homogeneity for $f$ gives us that $\bigcup n_{t}>\bigcup n_{s} \geq \beta$. Homogeneity for $f_{\omega \backslash Q^{\eta}}^{\eta}$ gives us that $\bigcup n_{t}=\bigcup n_{s^{\prime}}$, where

$$
s^{\prime}\left(\nu_{n}\right)= \begin{cases}t\left(\nu_{n}\right) & \text { for } n \in Q^{\eta} \\ x\left(\nu_{n}\right)\lceil\eta & \text { for } n \notin Q^{\eta}\end{cases}
$$

since $t=\left(s^{\prime},(t, u)_{Q^{\eta}}\right)_{\omega \backslash Q^{\eta}}$, for any $u>t$. Then we have $\beta \leq \bigcup n_{s^{\prime}}<\zeta$.

Finally, use $(k) \rightarrow(k)^{<\gamma}$ to obtain $q \in\left[q_{1}\right]^{k}$ homogeneous simultaneously for all $f_{\omega \backslash Q^{\eta}}, \eta<\gamma$. This satisfies our Claim and finishes the case: $\operatorname{cof}(\delta)>\bigcup k$.

Suppose now that $\operatorname{cof}(\delta)$ lies somewhere within the limits of the sequence. We can factor the partial ordering into a piece below $\operatorname{cof}(\delta)$ and a piece (at and) above. By Proposition 6.2, forcing first with the piece (at and) above either changes the cofinality of $\delta$ to $\gamma$ or leaves it unchanged (depending on whether $\operatorname{cof}(\delta)$ is an element of the sequence (at and) above). By Proposition 6.5, this forcing preserves the partition relation necessary to carry out the forcing on the piece below. All that remains is to show that after this first forcing, there is still no non-principal ultrafilter on $\omega$, so that the preceding proof applies.

Suppose that $p \Vdash$ " $\dot{U}$ is a non-principal ultrafilter on $\omega$." We can then define an ultrafilter $U^{*}$ in $\mathbf{V}$ as follows: For $x \subseteq \omega, x \in \mathbf{V}$, we say $x \in U^{*}$ iff $p_{0} \mid \sim$ " $x \in \dot{U}$." $U^{*}$ is definable in $\mathbf{V}$ and it is straightforward to show that it is a non-principal ultrafilter, contradicting our hypothesis.

\section{Finite SUPPORT}

Questions of choice limit us when dealing with products. One solution is to use inner models based on finite products. We exploit this to prove the following theorem.

Theorem 7.1. Suppose that $\mathbf{V}=L(\mathbb{R}) \vDash \mathrm{AD}$ and $A$ is a sequence of cardinals such that for all $k \in[A]^{<\omega},(k) \rightarrow(k)^{k}$. Let $h: A \rightarrow \Theta^{\mathbf{V}} \backslash A$ be any function in $\mathbf{V}$ such that for $\kappa \in A, h(\kappa)<\kappa$ and $h(\kappa)$ is a regular cardinal. Then there is a partial ordering $\mathcal{P} \in \mathbf{V}$ and a symmetric inner model $\mathbf{N}$ of $\mathrm{ZF}$ such that

(1) $\mathbf{V} \subseteq \mathbf{N} \subseteq \mathbf{V}^{\mathcal{P}}$,

(2) $\mathbf{N}$ and $\mathbf{V}$ contain the same cardinals, 
(3) $\Theta^{\mathbf{N}}=\Theta^{\mathbf{V}}$, and

(4) the cofinality function in $\mathbf{N}$ is given by:

$$
\operatorname{cof}(\kappa)= \begin{cases}h(\kappa) & \text { if } \operatorname{cof}^{\mathbf{V}}(\kappa) \in A \\ \operatorname{cof}^{\mathbf{V}}(\kappa) & \text { otherwise. }\end{cases}
$$

Proof. For convenience, we will write $\mathcal{P}_{\kappa}$ for $\mathcal{P}_{h(\kappa), \kappa}$ if $h(\kappa)>\omega$ and $\mathcal{P}_{\kappa}$ for $\mathcal{P}_{\omega \cdot \omega, \kappa}$ if $\kappa=\omega$. The partial ordering $\mathcal{P}$ with which we force to construct the model is all elements of $\prod_{\kappa \in A} \mathcal{P}_{\kappa}$ having finite support, i.e., those $p$ such that $\{\kappa \in A: p(\kappa) \neq$ $\langle\emptyset, \kappa\rangle\}$ is finite. We will denote the support of $p$ by $\operatorname{supp}(p)$. For any set $B \subseteq A$, we will write $\mathcal{P}_{B}$ for $\prod_{\kappa \in B} \mathcal{P}_{\kappa}$. The ordering on $\mathcal{P}_{B}$ is by component.

Let $G$ be $\mathbf{V}$-generic over $\mathcal{P}_{A}=\mathcal{P}$. The full generic extension fails to satisfy the theorem if $A$ is infinite. To define our model $\mathbf{N}$, we first define $G_{\kappa}$ for $\kappa \in A$ as the $\mathbf{V}$-generic object over $\mathcal{P}_{\kappa}$ generated by $G$, i.e., $q \in G_{\kappa}$ iff for some $p \in G$, $q=p(\kappa)$. By the Product Lemma, each $G_{\kappa}$ is $V$-generic over $\mathcal{P}_{\kappa}$ and for $k \in[A]^{<\omega}$, $\prod_{\kappa \in k} G_{\kappa}$ (which we will write as $G_{k}$ ) is $\mathbf{V}$-generic over $\mathcal{P}_{k}$. We can describe $\mathbf{N}$ as the least model of ZF extending $\mathbf{V}$ which contains, for each $\kappa \in A$, the set $G_{\kappa}$. More formally, let $\mathcal{L}_{1}$ be the sublanguage of the forcing language $\mathcal{L}$ with respect to $\mathcal{P}$ which contains symbols $\dot{v}$ for each $v \in \mathbf{V}$, a unary predicate symbol $\dot{\mathbf{V}}$ (to be interpreted $\dot{\mathbf{V}}(\dot{v})$ iff $v \in \mathbf{V}$ ), and symbols $\dot{G}_{\kappa}$ for each $\kappa \in A$. We can define $\mathbf{N}$ inside $\mathbf{V}[G]$ as follows:

$$
\begin{aligned}
& \mathbf{N}_{0}=\emptyset \\
& \mathbf{N}_{\alpha+1}=\left\{x \subseteq \mathbf{N}_{\alpha}: x \text { is definable by } \tau \in \mathcal{L}_{1} \text { of rank } \leq \alpha \text { over }\left\langle\mathbf{N}_{\alpha}, \in, c\right\rangle_{c \in \mathbf{N}_{\alpha}}\right\} \\
& \mathbf{N}_{\lambda}=\bigcup_{\alpha<\lambda} \mathbf{N}_{\alpha} \text { for } \lambda \text { a limit ordinal. } \\
& \mathbf{N}=\bigcup_{\alpha \in \mathrm{ORD}^{V}} \mathbf{N}_{\alpha} .
\end{aligned}
$$

Standard arguments show $\mathbf{N} \vDash Z F$ and if $A$ is finite, $\mathbf{N}=\mathbf{V}[G]$.

Lemma 7.2. Let $x$ and $y$ be sets with $x, y \in \mathbf{V}$, and let $f: x \rightarrow y$ be a function with $f \in \mathbf{N}$. Then $f \in \mathbf{V}\left[G_{k}\right]$, where $k \in[A]^{<\omega}$.

Proof. The proof is similar to that of Lemma 2.1 of $[\mathrm{A}]$. Let $p \in \mathcal{P}$ be so that $p \Vdash$ " $\dot{f}: x \rightarrow y$ is a function." Since $f \in \mathbf{N}$, we can choose $\dot{f} \in \mathcal{L}_{1}$, and $k \in[A]<\omega$, such that $\dot{f}$ mentions only terms of the form $\dot{G}_{\kappa}$ for $\kappa \in k$. By extending $p$ and/or $k$ if necessary, we can assume $\operatorname{supp}(p)=k$. We will identify $p$ with $p\lceil\operatorname{supp}(p)$, so we may consider $p$ both as a condition of $\mathcal{P}$ and as a condition of $\mathcal{P}_{k}$.

Thinking of $\dot{f}$ as a term for a set, define in $\mathbf{V}\left[G_{k}\right]$ the set

$$
g=\left\{\langle w, z\rangle \in \mathbf{V}: \exists q \in G_{k} \text { such that } q \Vdash p \text { and } q \Vdash_{\mathcal{P}} "\langle w, z\rangle \in \dot{f} "\right\} .
$$

We claim $p \Vdash " \dot{f}=\dot{g} "$. Certainly $p \Vdash " \dot{g} \subseteq \dot{f} "$. To show that $p \Vdash " \dot{f}=\dot{g} "$, suppose $q \Vdash p, q \in G$ with $q \Vdash$ " $\langle w, z\rangle \in \dot{f}$ ". We will be done if we can show that $q^{\prime}=q\lceil\operatorname{supp}(p) \Vdash "\langle w, z\rangle \in \dot{f} "$.

If $q^{\prime} \nVdash "\langle w, z\rangle \in \dot{f} "$, then choose $r \Vdash q^{\prime}, r \Vdash "\langle w, z\rangle \notin \dot{f} "$, with $\operatorname{supp}(q) \subseteq \operatorname{supp}(r)$. Extend $q$ to $s$ with $\operatorname{supp}(s)=\operatorname{supp}(r)$. Note that $s \Vdash "\langle w, z\rangle \in \dot{f} "$. We may assume that $s\lceil\operatorname{supp}(p)=r\lceil\operatorname{supp}(p)$.

By extending the appropriate sequence of ordinals as in Proposition 1.1 of [H2], we can assume $r(\kappa)_{0}$ and $s(\kappa)_{0}$ (the first coordinates) have the same length for all $\kappa$. 
Further, if we denote by $\operatorname{cl}(X)$ the $\omega$-closure of $X$, then $\operatorname{cl}\left(r(\kappa)_{1}\right) \cap \operatorname{cl}\left(s(\kappa)_{1}\right) \in[\kappa]^{\kappa}$ and $\left\langle\operatorname{cl}\left(r(\kappa)_{1}\right) \cap \operatorname{cl}\left(s(\kappa)_{1}\right)\right\rangle \subseteq\left\langle r(\kappa)_{1}\right\rangle \cap\left\langle s(\kappa)_{1}\right\rangle$. Using this, we may assume for all $\kappa \in \operatorname{supp}(r)$ that $r(\kappa)_{1}=s(\kappa)_{1}$. Now for $\kappa \in \operatorname{supp}(r)$ define $\pi_{\kappa}: \kappa \rightarrow \kappa$ by $\pi_{\kappa}\left(r(\kappa)_{0}(\xi)\right)=s(\kappa)_{0}(\xi)$ for each $\xi<\overline{s(\kappa)_{0}}$ and the identity otherwise. Define $\psi_{\kappa}: \mathcal{P}_{\kappa} \rightarrow \mathcal{P}_{\kappa}$ by $\psi_{\kappa}(x)=\left\langle\pi_{\kappa} \circ x_{0}, \pi_{\kappa}^{\prime \prime} x_{1}\right\rangle . \psi_{\kappa}$ is an isomorphism of the conditions in $\mathcal{P}_{\kappa}$ extending $r(\kappa)$ to the conditions in $\mathcal{P}_{\kappa}$ extending $s(\kappa)$. In addition, $\psi_{\kappa}(r(\kappa))=$ $s(\kappa)$.

Now define $\psi: \mathcal{P} \rightarrow \mathcal{P}$ by

$$
\psi(u)(\kappa)= \begin{cases}\psi_{\kappa}(u(\kappa)) & \text { if } \kappa \in \operatorname{supp}(r), \text { and } \\ u(\kappa) & \text { otherwise. }\end{cases}
$$

Note that for $\kappa \in \operatorname{supp}(p), \psi_{\kappa}$ is the identity, since $r(\kappa)=s(\kappa)$, hence $G_{\kappa}$ and $\dot{f}$ can be assumed to be invariant under $\psi$. Finally, $w$ and $z$, being (terms for) ground model sets, can also be assumed to be invariant under $\psi$ and so $s=\psi(r) \Vdash^{*} "\langle w, z\rangle \notin$ f'".

Since $s \Vdash^{*}\langle w, z\rangle \in \dot{f} "$, this is a contradiction and so $q^{\prime} \Vdash^{*}\langle w, z\rangle \in \dot{f} "$, i.e., $p \Vdash " \dot{f} \subseteq \dot{g} "$.

Our strategy is to reduce the problem to the finite case. Proposition 3.5, for example, tells us that cardinals are preserved in $\mathbf{V}^{\mathcal{P}_{k(n-1)}}$ and Proposition 6.3 tells us that $(k \uparrow(n-1)) \rightarrow(k \uparrow(n-1))^{k \uparrow(n-1)}$ is preserved as well. Working our way down, we see that all cardinals are preserved in $\mathbf{V}^{\mathcal{P}_{k}}$, for $k$ finite. Combining this with the previous lemma gives us (2) of the theorem. In a similar fashion, Fact 3.3 gives (4). To complete the proof, we need only the following:

Lemma 7.3. For any $\gamma \in A, \Theta^{\mathrm{V}}=\Theta^{\mathbf{V}^{\mathcal{P}_{\gamma}}}$.

Proof. Since $\gamma>\omega$ and $\mathbf{V}$ and $\mathbf{V}^{\mathcal{P}_{\gamma}}$ contain the same bounded subsets of $\gamma, \mathbb{R}^{\mathbf{V}}=$

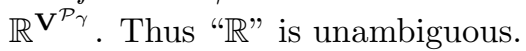

Suppose $\langle s, X\rangle \Vdash^{*} \dot{f}: \mathbb{R} \rightarrow \Theta^{\mathbf{V}}$ is onto." For each $r \in \mathbb{R}$ and each $t \in[X]^{<h(\gamma),}$ define in $\mathbf{V}$ :

$$
g(\langle r, t\rangle)= \begin{cases}\alpha & \text { if }(s \cup \omega t) \| \sim * \dot{f}(r)=\alpha " \\ 0 & \text { otherwise. }\end{cases}
$$

By the fact that any two conditions $\langle u, Y\rangle$ and $\left\langle u, Y^{\prime}\right\rangle$ are compatible (as in the proof of Lemma 7.2), $g$ is well-defined. Further, $g$ can easily be seen to be a surjection onto $\Theta^{\mathbf{V}}$. Since any pair $\langle r, t\rangle$ as above codes a subset of $\gamma, g$ can be used to define in $\mathbf{V}$ a mapping $h$ of $2^{\gamma}$ onto $\Theta^{\mathbf{V}}$. Since $\gamma<\Theta^{\mathbf{V}}$, however, there is a pre-well-ordering of $\mathbb{R}$ in $\mathbf{V}$ of size $\gamma$. We can use the Coding Lemma (see [Mo2]) to produce a mapping $k$ from $\mathbb{R}$ onto $2^{\gamma}$, and then $h \circ k$ is a mapping in $\mathbf{V}$ from $\mathbb{R}$ onto $\Theta^{\mathbf{V}}$, a contradiction.

Note that the only differences between the cofinality function in $\mathbf{V}$ and $\mathbf{N}$ are those that are forced to occur. Thus, since $\Theta$ is regular in $\mathbf{V}$ (see [Mo2]), Lemma 7.3 implies $\Theta$ is regular in $\mathbf{N}$.

Note also that Theorem 7.1 does not require that $(A) \rightarrow(A)^{A}$.

Note that if $A$ is infinite, then as in $[\mathrm{A}], \mathbf{N} \not \models \mathrm{AC}_{\omega}$. To see this, suppose $k \in[A]^{\omega}$. For all $i<\omega$, let $X(i)=\left\{G: G\right.$ is $\mathbf{V}$-generic over $\left.\mathcal{P}_{k(i)}\right\}$. The collection $\{X(i)\}_{i<\omega}$ 
is in $\mathbf{N}$, but has no choice function $f$. If $f$ is in the cartesian product, then by identifying generic sets with cofinal sequences, $f$ can be coded by a set of ordinals. By Lemma 7.2, $f$ can be obtained by forcing over $\mathbf{V}$ using only a finite number of Magidor-like orderings. Such an extension, however, only destroys the regularity of finitely-many $k(i)$, while $f$ witnesses the singularity of all the $k(i)$, a contradiction.

On the other hand, if $A$ is finite and each $\gamma \in A$ has its cofinality changed to some uncountable cardinal, then $\mathbf{N} \vDash D C$, since $\mathbf{N}=\mathbf{V}[G], \mathbf{V} \vDash D C$ (recall that assuming $\mathrm{AD}, L(\mathbb{R}) \vDash \mathrm{DC}$ ), and $\mathcal{P}$ is countably closed. If, however, some successor cardinal $\kappa \in A$ has its cofinality changed to $\omega$, then regardless of the size of $A$, $\mathbf{N} \not \models \mathrm{AC}_{\omega}$.

\section{Countable support}

In this section, we present a general theorem in which a certain polarized partition property holding for a set $A$ of cardinals is used to change the cofinality of each member of $A$ to $\omega_{1}$ and preserve the fact that they are cardinals. We then apply this theorem (in section 11) to derive the existence of the model mentioned in the Introduction which provides a partial answer to Woodin's question on the consistency of the theory "ZF $+\mathrm{DC}+\omega_{1}$ is the only regular uncountable cardinal." The challenge is to preserve DC and the method is to move to countable support.

The general theorem we prove is

Theorem 8.1. Suppose that $\mathbf{V}=L(\mathbb{R}) \vDash A D$ and $A \subseteq \Theta^{\mathbf{V}}, A \in \mathbf{V}$, is such that all $k \in[A]^{<\omega_{1}}$ satisfy $(k) \rightarrow(k)^{<\omega_{1}}$. Then there is a partial ordering $\mathcal{P} \in \mathbf{V}$ and an inner model $\mathbf{N}$ of $\mathrm{ZF}$ such that

(1) $\mathbf{V} \subseteq \mathbf{N} \subseteq \mathbf{V}^{\mathcal{P}}$

(2) $\Theta^{\mathbf{N}}=\Theta^{\bar{v}}$,

(3) $\mathbf{N} \vDash$ "ZF $+\mathrm{DC}+\kappa$ is a cardinal of cofinality $\omega_{1}$ for all $\kappa \in A$," and

(4) for all cardinals $\delta$ of $\mathbf{V}$, $\operatorname{cof}^{\mathbf{N}}(\delta)=\operatorname{cof}^{\mathbf{V}}(\delta)$ if $\operatorname{cof}^{\mathbf{V}}(\delta) \notin A$ and $\operatorname{cof}^{\mathbf{N}}(\delta)=\omega_{1}$ otherwise.

Proof. The proof has similarities with the proof of Theorem 7.1. For each $\kappa \in A$, let $\mathcal{P}_{\kappa}=\mathcal{P}_{\omega_{1}, \kappa}$. The partial ordering $\mathcal{P}$ we use now will, as before, be a subset of $\mathcal{P}_{A}$. This time, however, $\mathcal{P}$ will be composed of all elements having countable support. The ordering on $\mathcal{P}$ is once again componentwise.

As before, we may assume our conditions have first coordinates of equal length in all non-trivial components.

Now let $G$ be $\mathbf{V}$-generic over $\mathcal{P}$. For $\kappa \in A$, let $G_{\kappa}$ be the $\mathbf{V}$-generic object over $\mathcal{P}_{\kappa}$ generated by $G$, i.e., $q \in G_{\kappa}$ iff for some $p \in G, q=p(\kappa)$. The Product Lemma again implies that for $k \in[A]^{<\omega_{1}}, G_{k}=\prod_{\kappa \in k} G_{\kappa}$ is $\mathbf{V}$-generic over $\mathcal{P}_{k}$. This allows us to describe $\mathbf{N}$ intuitively as the least model of ZF extending $\mathbf{V}$ which contains, for each $k \in[A]^{<\omega_{1}}$, the set $G_{k}$. More formally, let $\mathcal{L}_{1}$ be the sublanguage of the forcing language $\mathcal{L}$ with respect to $\mathcal{P}$ which contains symbols $\dot{v}$ for each $v \in \mathbf{V}$, a unary predicate symbol $\dot{\mathbf{V}}$ (to be interpreted $\dot{\mathbf{V}}(\dot{v})$ iff $v \in \mathbf{V}$ ), and symbols $\dot{G}_{k}$ for each $k \in[A]^{<\omega_{1}}$, which are interpreted as $G_{k}$. $\mathbf{N}$ can then be defined inside $\mathbf{V}[G]$ 
as follows:

$$
\begin{aligned}
& \mathbf{N}_{0}=\emptyset . \\
& \mathbf{N}_{\alpha+1}=\left\{x \subseteq \mathbf{N}_{\alpha}: x \text { is definable by } \tau \in \mathcal{L}_{1} \text { of rank } \leq \alpha \text { over }\left\langle\mathbf{N}_{\alpha}, \in, c\right\rangle_{c \in \mathbf{N}_{\alpha}}\right\} \\
& \mathbf{N}_{\lambda}=\bigcup_{\alpha<\lambda} \mathbf{N}_{\alpha} \text { for } \lambda \text { a limit ordinal. } \\
& \mathbf{N}=\bigcup_{\alpha \in \mathrm{ORD}^{V}} \mathbf{N}_{\alpha} .
\end{aligned}
$$

Standard arguments show $\mathbf{N} \vDash \mathrm{ZF}$ and if $A$ is countable, $\mathbf{N}=\mathbf{V}[G]$.

Lemma 8.2. Let $x$ and $y$ be sets with $x, y \in \mathbf{V}$ and let $f: x \rightarrow y$ be a function with $f \in \mathbf{N}$. Then $f \in \mathbf{V}\left[G_{k}\right]$, for some $k \in[A]<\omega_{1}$.

Proof. The proof is virtually identical to the proof of Lemma 7.2. We note that by a coding argument, a term $\dot{f}$ for $f$ can be assumed to mention only one term of the form $\dot{G}_{k}$, where $k \in[A]^{<\omega_{1}}$. Once this has been done, the only difference in the proofs is that the supports used now are countable, not finite as in Lemma 7.2. However, since we can extend the first coordinates of conditions canonically, and since the union of two countable supports is also a countable support, all aspects of the proof of the current lemma can be carried out as before.

Lemma 8.3. $\mathrm{N} \vDash \mathrm{DC}$.

Proof. Let $p$ force:

$$
\text { " } \dot{R} \subseteq \dot{X} \times \dot{X}, \dot{R}, \dot{X} \in \dot{\mathbf{N}}, \dot{X} \neq \emptyset \text {, and } \forall x \in \dot{X} \exists y \in \dot{X}\langle x, y\rangle \in \dot{R} . "
$$

Using the fact that $\mathbf{V}=L(\mathbb{R}) \vDash \mathrm{DC}$ since $\mathbf{V}=L(\mathbb{R}) \vDash \mathrm{AD}$ (see $[\mathrm{Ke} 2]$ ), we can define inductively a sequence of conditions $\left\langle p_{n}: n<\omega\right\rangle$ and a sequence of terms $\left\langle\tau_{n}: n<\omega\right\rangle$ so that

(1) each $\tau_{i} \in \mathcal{L}_{1}$,

(2) $p_{0} \Vdash p$,

(3) $p_{0} \Vdash " \tau_{0} \in \dot{X} "$, and

(4) for $n>0, p_{n} \Vdash p_{n-1}$ and $p_{n} \Vdash "\left\langle\tau_{n-1}, \tau_{n}\right\rangle \in \dot{R}$."

Since $\mathbf{V} \vDash D C$ and $\mathbf{V} \vDash$ " $\mathcal{P}_{\kappa}$ for $\kappa \in A$ is countably closed," the definition of $\mathcal{P}$ ensures that $\mathbf{V} \vDash$ " $\mathcal{P}$ is countably closed." This means there is a single $q$ extending every $p_{n}$, so that for any $n<\omega, q \Vdash^{"}\left\langle\tau_{n-1}, \tau_{n}\right\rangle \in \dot{R}$." By $\mathrm{AC}_{\omega}, \mathbf{V} \vDash$ "The countable union of countable sets is countable," so $\bigcup_{n<\omega} \operatorname{supp}\left(\tau_{n}\right)$ is countable and can be used as a support to define a term $\tau \in \mathcal{L}_{1}$ for $\left\langle\tau_{n}: n<\omega\right\rangle$. Thus, $q \Vdash$ " $\tau \in \dot{\mathbf{N}}$ is a witness for DC for $\dot{R}$."

Lemma 8.4. $\mathbf{N} \vDash$ "If $\gamma \in A, \gamma$ is a cardinal." Further, for all cardinals $\delta$ of $\mathbf{V}$, $\operatorname{cof}^{\mathbf{N}}(\delta)=\operatorname{cof}^{\mathbf{V}}(\delta)$ if $\operatorname{cof}^{\mathbf{V}}(\delta) \notin A$ and $\operatorname{cof}^{\mathbf{N}}(\delta)=\omega_{1}$ otherwise.

Proof. Since $\mathrm{ZF}+\mathrm{AD} \vdash$ "All sets of reals are Lebesgue--measurable" and a nonprincipal ultrafilter on $\omega$ is a non-Lebesgue-measurable set, $\mathbf{V} \vDash$ "There is no non-principal ultrafilter on $\omega$." Therefore, by Proposition 6.6 and Lemma 8.2, the cofinality is what it should be.

For the cardinality, fix $\gamma \in A$. By Lemma 8.2, if $\zeta<\gamma$ and $f: \zeta \rightarrow \gamma$ is a function, $f \in \mathbf{N}$, then $f \in \mathbf{V}\left[G_{k}\right]$ for some $k \in[A]^{<\omega_{1}}$. Since $\mathbf{V}\left[G_{k}\right] \subseteq \mathbf{N}$, it suffices to show that for any $k \in[A]^{<\omega_{1}}, \mathbf{V}\left[G_{k}\right] \vDash " \gamma$ is a cardinal." 
Fix $k \in[A]^{<\omega_{1}}, k \in \mathbf{V}$, and assume without loss of generality that $\gamma \in k$. Let $k_{0}$ be the part of $k$ below $\gamma$ and $k_{1}$ the part of $k$ at and above $\gamma$. By Proposition 6.4, $\mathbf{V}^{\mathcal{P}_{k_{0}}} \vDash\left(k_{1}\right) \rightarrow\left(k_{1}\right)^{<\omega_{1}}$, so by Fact $4.5, \mathbf{V}^{\mathcal{P}_{k_{0}}} \vDash \gamma \rightarrow(\gamma)^{<\omega_{1}}$. Hence, $\gamma$ is still a cardinal. By Proposition 6.2, forcing with $\mathcal{P}_{k_{1}}$ over $\mathbf{V}^{\mathcal{P}_{k_{0}}}$ adds no new bounded subsets of $k_{1}(0)=\gamma$. Hence, $\gamma$ is still a cardinal in $\left(\mathbf{V}^{\mathcal{P}_{k_{0}}}\right)^{\mathcal{P}_{k_{1}}}=\mathbf{V}^{\mathcal{P}_{k}}$.

Lemma 8.5. $\Theta^{\mathbf{N}}=\Theta^{\mathrm{V}}$.

Proof. The proof is similar in spirit and method to the proof of Lemma 7.3. By Lemma 8.2, any $x \subseteq \omega$ lies in some $\mathbf{V}\left[G_{k}\right]$ for some $k \in[A]^{<\omega_{1}}$. Since all members of $k$ are uncountable and since $\mathbf{V}$ and $\mathbf{V}\left[G_{k}\right]$ have the same bounded subsets of $k(0), x \in \mathbf{V}$. This means that $\mathbb{R}^{\mathbf{N}}=\mathbb{R}^{\mathbf{V}}$. We will therefore once more write $\mathbb{R}$ unambiguously.

Suppose now that $f \in \mathbf{N}$ maps $\mathbb{R}$ onto $\Theta^{\mathbf{V}}$. Using Lemma 8.2, choose $k$ so that $f \in \mathbf{V}\left[G_{k}\right], k \in[A]^{<\omega_{1}}$, and suppose $p \in \mathcal{P}_{k}$ forces " $\dot{f}: \mathbb{R} \rightarrow \Theta^{\mathbf{V}}$ is surjective." For each $r \in \mathbb{R}$ and $t \in\left[p_{1}\right]^{<\omega_{1}}$, define

$$
g(\langle r, t\rangle)= \begin{cases}\alpha & \text { if }\left(p_{0} \frown \omega t\right) \| \sim \sim \dot{f}(r)=\alpha ", \\ 0 & \text { otherwise. }\end{cases}
$$

$g$ is well-defined and the range is all of $\Theta^{\mathbf{V}}$. Since $\Theta$ is regular in $\mathbf{V}$ and $k$ is countable, $\bigcup k=\lambda<\Theta^{\mathbf{V}}$, so any $t$ as above is coded by a subset of $\lambda$. The proof now finishes in the manner of the proof of Lemma 7.3, with first a map in $\mathbf{V}$ from $\mathbb{R}$ onto $2^{\lambda}$ and then a map onto $\Theta^{\mathbf{V}}$, producing a contradiction.

We remark that since $\mathbf{V} \vDash$ " $\Theta$ is regular", Lemma 8.4 and Lemma 8.5 imply $\mathbf{N} \vDash$ " $\Theta$ is regular" as well.

\section{INCLUDING ULTRAPOWERS}

In our chosen universe, not every regular cardinal is a member of a sequence satisfying $(k) \rightarrow(k)^{k}$.

If $\mu$ is a normal measure on $\kappa$, we will use $\kappa_{\mu}$ to represent the ultrapower, $\kappa^{\kappa} / \mu$, of $\kappa$ via $\mu$. These cardinals satisfy powerful partition properties but fall short of being strong partition cardinals. The following is due to E. M. Kleinberg.

Fact 9.1. If $\kappa$ satisfies $\kappa \rightarrow(\kappa)^{\kappa}$, then $\kappa_{\mu} \rightarrow\left(\kappa_{\mu}\right)^{\delta}$, for all $\delta<\omega_{1}$. Further, if $\kappa^{\kappa} / \mu=\kappa^{+}$, then $\kappa_{\mu} \rightarrow\left(\kappa_{\mu}\right)^{\delta}$, for all $\delta<\kappa_{\mu}$. At the same time, $\kappa_{\mu} \nrightarrow\left(\kappa_{\mu}\right)^{\kappa_{\mu}}$. (See [Kl1], [Kl2].)

This implies that if $\mu$ is a normal measure on $\kappa \in k$ and $(k) \rightarrow(k)^{k}$, then $\kappa_{\mu}$ cannot be in any sequence satisfying the same property. Under AD, many regular cardinals are of this sort. Ultrapowers can, however, be in a sequence of less extravagant power.

In this section, we assume DC throughout.

Proposition 9.2. Suppose $(k) \rightarrow(k)_{\omega_{1}}^{k}$ and $k^{+}$is a countable sequence composed of members of $k$ and ultrapowers of members of $k$. Then $\left(k^{+}\right) \rightarrow\left(k^{+}\right)^{<\omega_{1}}$.

Proof. We will need some of the extensive machinery developed by Kleinberg. Familiarity with his work is useful, but not necessary.

For $\kappa$ measurable, $\mu$ a normal measure on $\kappa$, and for $p \in[\kappa]^{\kappa}$, let $\ulcorner p\urcorner^{\mu}$ be the ordinal below $\kappa_{\mu}$ represented by the increasing sequence $p$. Let $S_{\mu}^{p}=\left\{\ulcorner q\urcorner^{\mu}\right.$ : $\left.q \in[p]^{\kappa}\right\}$. Central to Kleinberg's work are the "break" and "shuffle" functions: 
$b k_{\zeta}:[\kappa]^{\kappa} \rightarrow\left[[\kappa]^{\kappa}\right]^{\zeta}$ and $s h_{\zeta}:\left[[\kappa]^{\kappa}\right]^{\zeta} \rightarrow[\kappa]^{\kappa}$, for all $\zeta<\kappa^{+}$. We list some of his results.

Fact 9.3. Assume $\mu$ is a normal measure on $\kappa$. Then

a) For all $\alpha<\zeta<\kappa^{+}, p \in[\kappa]^{\kappa},\left\ulcorner b k_{\zeta}(p)(\alpha)\right\urcorner^{\mu}=S_{\mu}^{p}(\alpha)$.

b) For all $\zeta<\kappa^{+}, p \in[\kappa]^{\kappa}, \operatorname{sh}_{\zeta}\left(b k_{\zeta}(p)\right)=p$.

c) For all $\alpha<\zeta<\kappa^{+},\left\{p_{\delta}\right\}_{\delta<\zeta} \in\left[[\kappa]^{\kappa}\right]^{\zeta},\left\ulcorner b k_{\zeta}\left(s h_{\zeta}\left(\left\{p_{\delta}\right\}_{\delta<\zeta}\right)\right)(\alpha)\right\urcorner^{\mu}=\left\ulcorner p_{\alpha}\right\urcorner^{\mu}$.

Now suppose we are given $f:\left[k^{+}\right]^{\delta} \rightarrow 2, \delta<\omega_{1}$. Let $g_{\delta}:[k]^{k} \rightarrow 2$ be defined by $g_{\delta}(W)=f\left(W^{(\delta)}\right)$, where

$$
W^{(\delta)}(\kappa)= \begin{cases}W(\kappa)\lceil\delta & \text { if } \kappa \in k, \\ \left\{\left\ulcorner b k_{\delta}(W(\gamma))(\alpha)\right\urcorner \mu\right\}_{\alpha<\delta} & \text { if } \kappa=\gamma_{\mu}, \gamma \in k .\end{cases}
$$

Let $X \in[k]^{k}$ be homogeneous for $g_{\delta}$. Then $X^{\prime} \in\left[k^{+}\right]^{k^{+}}$is homogeneous for $f$, where

$$
X^{\prime}(\kappa)= \begin{cases}X(\kappa) & \text { if } \kappa \in k \\ S_{\mu}^{X(\gamma)} & \text { if } \kappa=\gamma_{\mu}, \gamma \in k\end{cases}
$$

since if $Y \in\left[X^{\prime}\right]^{\delta}$, we can construct $W \in[X]^{k}$ such that $W^{(\delta)}=Y$ as follows: There is no difficulty in dealing with a cardinal $\gamma$ and its ultrapower at the same time (the value of $\left\{\left\ulcorner b k_{\delta}(W(\gamma))(\alpha)\right\urcorner^{\mu}\right\}_{\alpha<\delta}$ is independent of $W(\gamma)\lceil\delta$ ), but there is a difficulty when we have several measures on a single $\gamma$. For this we use a lemma.

Lemma 9.4. Given $\left\{\mu_{n}\right\}_{n \in \omega}$, normal measures on $\kappa, p \in[\kappa]^{\kappa}$, and $q_{n} \in\left[S_{\mu_{n}}^{p}\right]^{\delta}$, $\delta<\omega_{1}$, there exists $r \in[p]^{\kappa}$ such that for each $n, q_{n}=\left\ulcorner b k_{\delta}(r)\right\urcorner \mu_{n}$.

Proof. We first find $r_{0} \in[p]^{\kappa}$ such that $q_{n}(0)=\left\ulcorner r_{0}\right\urcorner \mu_{n}$ for all $n$. Begin by partitioning $\kappa$ into disjoint sets, $\kappa=\bigcup_{n \in \omega} A_{n}$ such that for each $n, \mu_{n}\left(A_{n}\right)=$ 1. Choose for each $n t_{n} \in[p]^{\kappa}$ such that $\left\ulcorner t_{n}\right\urcorner^{\mu_{n}}=q_{n}(0)$. Now set $r_{0}(\alpha)=$ $\bigcup_{\beta<\alpha} r_{0}(\beta) \cup t_{n}(\alpha)$, where $\alpha \in A_{n}$. The set, $\left\{\alpha \mid \bigcup_{\beta<\alpha} r_{0}(\beta)=\alpha\right\}$, is closed and unbounded (closed is obvious; for unbounded, note that for any $\alpha$, the sup of $r_{0}(\alpha), r_{0}\left(r_{0}(\alpha)\right), r_{0}\left(r_{0}\left(r_{0}(\alpha)\right)\right), \ldots$ is in the set) and consequently for $\mu_{n}$-measure one many $\alpha, r_{0}(\alpha)=t_{n}(\alpha)$, and so $\left\ulcorner r_{0}\right\urcorner \mu_{n}=\left\ulcorner t_{n}\right\urcorner \mu_{n}=q_{n}(0)$. To complete the proof, we do this for $r_{\xi}$ and $q_{n}(\xi)$ for all $\xi<\delta$ and set $r=s h_{\delta}\left(\left\{r_{\xi}\right\}_{\xi<\delta}\right)$.

Returning to our task, from $(k) \rightarrow(k)^{k}$ we infer $\left(k^{+}\right) \rightarrow\left(k^{+}\right)^{\delta}$. Given now a partition $f:\left[k^{+}\right]^{<\omega_{1}} \rightarrow 2$, we can define an auxiliary partition $g:[k]^{k} \rightarrow \omega_{1}+1$ by $g(W)=\delta$ iff $\delta$ is least such that $W$ is not homogeneous for $g_{\delta}$. Let $X \in[k]^{k}$ be homogeneous for $g$. The range of $g$ on $[X]^{k}$ cannot be $\delta<\omega_{1}$, since we can always find $Y \in[X]^{k}$ homogeneous for a particular $g_{\delta}$. Thus $X^{\prime}$, as defined above, is homogeneous for $f$.

The extra assumption of the subscript poses no problem for our applications. The following proposition gives us this, assuming $\omega_{1}^{\omega_{1}} / \mu=\omega_{2}$.

Proposition 9.5. If $\left(\omega_{1} \frown k\right) \rightarrow\left(\omega_{1} \frown k\right)^{\omega_{1} \frown k}$ and $\omega_{1}^{\omega_{1}} / \mu=\omega_{2}, \mu$ normal on $\omega_{1}$, then $\left(\omega_{2} \frown k\right) \rightarrow\left(\omega_{2} \frown k\right)_{\omega_{1}}^{\omega_{1} \frown k}$.

Proof. Suppose $f:\left[\omega_{2} \frown k\right]^{\omega_{1} \frown k} \rightarrow \omega_{1}$. For $W \in\left[\omega_{1} \frown k\right]^{\omega_{1} \frown k}$, we will write $W^{*}$ for all but the first component of $W$, that is, $W=W(0)-W^{*}$. Now define, for all $\delta<\omega_{1}, f_{\delta}:\left[\omega_{2} \frown k\right]^{\omega_{1} \frown k} \rightarrow 2$ and $g:\left[\omega_{1} \frown k\right]^{\omega_{1} \frown k} \rightarrow 2$ by $f_{\delta}(X)=0$ iff $f(X)<\delta$, and $g(W)=0$ iff $f_{\delta}$ is constant on $\left\{S_{\mu}^{W(0)}\left\lceil\omega_{1}\right\} \times\left[W^{*}\right]^{k}\right.$ for all $\delta<W(0)(0)$. Let 
$X \in\left[\omega_{1} \frown k\right]^{\omega_{1} \frown k}$ be homogeneous for $g$. We must have $g^{\prime \prime}[X]^{\omega_{1} \frown k}=\{0\}$ since we can use Fact 4.9 to find $Y \in\left[X^{*}\right]^{k}$ so that $\left\{\left(X(0)^{\omega_{1}} / \mu\right)\left\lceil\omega_{1}\right\} \times[Y]^{k}\right.$ is homogeneous simultaneously for $f_{\delta}$ for all $\delta<X(0)(0)$. We then have $g(X(0), Y)=0$. Note that if $W \in[X]^{\omega_{1}} \smile^{k}$, then $f_{\delta}$ is actually constant on $\left\{S_{\mu}^{W(0)}\left\lceil\omega_{1}\right\} \times\left[W^{*}\right]^{k}\right.$ for all $\delta<\omega_{1}$ since $g\left(W(0) \backslash \alpha, W^{*}\right)=0$ for all $\alpha<\omega_{1}$, and $S_{\mu}^{W(0)}=S_{\mu}^{W(0) \backslash \alpha}$. Consequently, $f$ itself is constant on $\left\{S_{\mu}^{W(0)}\left\lceil\omega_{1}\right\} \times\left[W^{*}\right]^{k}\right.$.

With Kleinberg's machinery, any sequence $t \in\left[S_{\mu}^{X(0)}\right]^{\omega_{1}}$ can be written as $S_{\mu}^{Z} \uparrow$ $\omega_{1}$ for some $Z \in[X(0)]^{\omega_{1}}$ (since $\omega_{1}^{\omega_{1}} / \mu=\omega_{2}$, we can break $X(0)$ up into a sequence long enough to contain representatives for each member of $t$, which can then be shuffled together to form $Z$ ). Thus, $f$ induces a partition of $\left[S_{\mu}^{X(0)}\right]^{\omega_{1}}$ into $\omega_{1}$. Using Fact 9.1 and Fact 1.7, we can find $T \in\left[S_{\mu}^{X(0)}\right]^{\omega_{2}}$ such that $f$ is constant on $[T]^{\omega_{1}} \times\left[X^{*}\right]^{k}$. We then have that $T \frown X^{*}$ is homogeneous for $f$.

We can strengthen Proposition 9.2.

Corollary 9.6. Given $\left(\omega_{1} \frown k\right) \rightarrow\left(\omega_{1} \frown k\right)^{\omega_{1} \frown k}$ and $\omega_{1}^{\omega_{1}} / \mu=\omega_{2}$ and $k^{+}$, a countable sequence composed of members of $k$ and ultrapowers of members of $k$, then $\left(\omega_{2} \frown k^{+}\right) \rightarrow\left(\omega_{2} \frown k^{+}\right)<\omega_{1}$.

Proof. Fact 4.9 gives us $\left(\omega_{1} \frown k\right) \rightarrow\left(\omega_{1} \frown k\right)_{\omega}^{\omega_{1}} \frown^{k}$. From this, we can find homogeneous sets for all partitions $F:\left[\omega_{1} \frown k\right]^{\omega_{1} \frown k} \rightarrow \omega_{1}$ which are independent of initial segments on the first coordinate, that is, for $F$ satisfying $F(p-X)=$ $F\left(\left(p \nmid\left(\omega_{1} \backslash \alpha\right) \frown X\right)\right.$, for $p \in\left[\omega_{1}\right]^{\omega_{1}}, \alpha<\omega_{1}$. (Find $q \frown Y$ homogeneous for $G:\left[\omega_{1} \frown k\right]^{\omega_{1} \frown k} \rightarrow 2$ defined by $G(p \frown X)=0$ iff $F(p \frown X)<p(0)$, then apply $\left(\omega_{1} \frown k\right) \rightarrow\left(\omega_{1} \frown k\right)_{\omega}^{\omega_{1} \frown k}$.) This is sufficient to follow the proof of Proposition 9.2 .

Combining Corollary 9.6 and Proposition 6.6, we can prove:

Proposition 9.7. Assume the hypotheses of Corollary 9.6 and suppose that there is no non-principal ultrafilter on $\omega$. Then for all cardinals $\delta$ of $\mathbf{V}, \operatorname{cof}^{\mathbf{V}^{\mathcal{P}} \omega_{1}, \omega_{2}-{ }^{+}}(\delta)=$

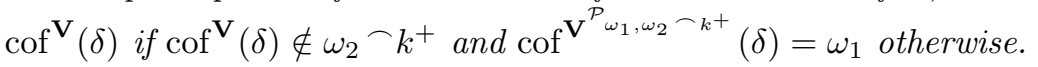

Proposition 9.8. If $\kappa>\omega_{1}$ satisfies $\kappa \rightarrow(\kappa)_{\omega_{1}}^{\kappa}$, or $\kappa=\omega_{1}$ satisfies $\kappa \rightarrow(\kappa)^{\kappa}$ and $\mu$ is a normal measure on $\kappa$ with $\kappa^{\kappa} / \mu=\kappa^{+}$, then forcing with $\mathcal{P}_{\omega_{1}, \kappa_{\mu}}$ preserves all cardinals.

Proof. By Proposition 9.1 and Fact 4.5, if $\kappa>\omega_{1}$, we have $\kappa_{\mu} \rightarrow\left(\kappa_{\mu}\right)^{<\omega_{1}}$. If $\kappa=\omega_{1}$, Fact 1.5 and Fact 9.1 give us $\kappa_{\mu} \rightarrow\left(\kappa_{\mu}\right)^{<\omega_{1}}$. With this, no bounded subsets of $\kappa_{\mu}$ are added and hence all cardinals $\leq \kappa_{\mu}$ are preserved.

Now suppose $\kappa_{\mu} \leq \delta<\zeta$ and $p \Vdash " \dot{f}: \delta \rightarrow \zeta$, onto", $p \in \mathcal{P}_{\omega_{1}, \kappa_{\mu}}$. Define $n_{s}$ and $n_{t}$, for $s \in\left[\kappa_{\mu}\right]^{<\omega_{1}}$ and $t \in\left[\kappa_{\mu}\right]^{\kappa_{\mu}}$ as in the proof of Proposition 3.5. As before, $\left\|n_{t}\right\|<\zeta$.

Define $h:[\kappa]^{\kappa} \rightarrow \zeta$ by: $h(t)=\bigcap\left(\zeta \backslash n_{S_{\mu}^{t}}\right)$. As before, we can find $q \in[\kappa]^{\kappa}$ so that $r, r^{\prime} \in[q]^{\kappa}, r^{\prime} \subseteq r$ imply $h(r) \leq h\left(r^{\prime}\right)$. In addition, we can ensure that $S_{\mu}^{q}$ is thinner than $p_{1}$, so that $\left\langle p_{0}, S_{\mu}^{q}\right\rangle$ extends $p=\left\langle p_{0}, p_{1}\right\rangle$ (for example, if $q$ is homogeneous for the partition: $F(x)=0$ iff there is a member of $p_{1}$ between $b k_{2}(x)(0)$ and $\left.b k_{2}(x)(1)\right)$.

Let $\beta=h(q)$. Choose $s \in\left[S_{\mu}^{q}\right]^{\xi}$ for some $\xi<\omega_{1}$ with $\beta \in n_{s}$. We wish to proceed as before but there is a difficulty. We want to take the part of $S_{\mu}^{q}$ above 
$\bigcup s$, then add $s$ as in the previous proof. The problem is that we are working at the $\kappa$-level. If we thin $q$ to $r$ so that the least element of $S_{\mu}^{r}$ is above $s$, it may not be true that $h(r) \leq h(q)$, that is, intervals of $S_{\mu}^{r}$ are not necessarily intervals of $S_{\mu}^{q}$ since the former is not a final segment of the latter.

To deal with this, choose, for each $\alpha<\xi, v_{\alpha} \in[q]^{\kappa}$ such that $\left\ulcorner v_{\alpha}\right\urcorner=s(\alpha)$. Let $w=\operatorname{sh}_{\xi}\left(\left\langle v_{\alpha}: \alpha<\xi\right\rangle\right)$, so that $\left\ulcorner b k_{\xi}(w)\right\urcorner=s$. Now form $t, r \in[q]^{\kappa}$ as follows: place the first $\xi$-many elements of $w$ in $t$. Next place the first element of $q$ above $t$ in $r$. In general, at stage $\alpha$, place the first $\xi$ elements of $w$ above $r$ so far defined in $t$ and then place the first $\rho$ elements of $q$ above this in $r$, where $\rho$ is the least indecomposable greater than $\alpha$ (indecomposable means that the sum of ordinals less that $\rho$ is again less than $\rho)$. Since $q \supseteq t \cup r \supseteq r, h(q) \leq h(t \cup r) \leq h(r)$. Kleinberg's methods give us that $\left\ulcorner b k_{\xi}(t \cup r)\right\urcorner=\left\ulcorner b k_{\xi}(t)\right\urcorner=\left\ulcorner b k_{\xi}(w)\right\urcorner=s$ (since for a closed, unbounded set of ordinals $\alpha, t, t \cup r$, and $w$ all have the same $\alpha^{t h}$ element and the same $(\alpha+\eta)^{t h}$ elements for all $\eta<\xi$ ), so that $\beta \in n_{S_{\mu}^{t u r}}$ (and so $h(t \cup r) \neq \beta)$.

Claim. Every interval of $S_{\mu}^{r}$ of length less than $\omega_{1}$ is also an interval of $S_{\mu}^{q}$. This claim completes the proof, since the definition of $h$ and $n_{t}$ will then give us $h(r) \leq$ $h(q)$, so $h(t \cup r)=h(q)=\beta$, a contradiction.

Proof of the claim. Suppose that $x$ is a $\tau$-sequence from $S_{\mu}^{r}, \tau<\omega_{1}$, forming an interval in $S_{\mu}^{r}$. Let $a \in[\kappa]^{\kappa}$ be such that $x(0)$ is represented by $r$ composed with a. For all $\sigma<\tau$, the $\kappa$-sequence $\{r(a(\alpha)+\sigma)\}_{\alpha<\kappa}$ represents $x(\sigma)$. But for each $\alpha>\tau, r(a(\alpha))$ is in a sequence of members of $q$ of indecomposable length greater than $\tau$, hence $r(a(\alpha)+\sigma)$ is an element of $q$ and so $x=\left\ulcorner b k_{\tau}(r \circ a)\right\urcorner$ is an interval of $S_{\mu}^{q}$.

\section{INCLUDING ULTRAPRODUCTS}

Ultraproducts of members of partition sequences can be included as well. We assume DC throughout this section.

If $\mu$ is a countably additive measure on $\kappa$ and $k$ is a $\kappa$-sequence of cardinals, we will use $k_{\mu}$ to represent the ultraproduct, $\left[\prod_{\alpha \in k} \alpha\right] / \mu$. For $q \in \prod_{\alpha \in k} \alpha$, we will write $\ulcorner q\urcorner^{\mu}$ for the ordinal corresponding to $q$ in $\left[\prod_{\alpha \in k} \alpha\right] / \mu$. If $X \in[k]^{k}$, we will abbreviate $\left[\prod_{\alpha \in k} X(\alpha)\right] / \mu$ by $\Pi_{\mu}^{X}$.

Proposition 10.1. If $k$ satisfies $(k) \rightarrow(k)^{\delta}, \mu$ a countably additive measure on $\operatorname{len}(k), \delta<k(0)$, then

$$
k_{\mu} \rightarrow\left(k_{\mu}\right)^{\delta}
$$

Proof. Given a partition $f$ on $\left[k_{\mu}\right]^{\delta}$, define $g$ on $[k]^{\delta}$ by $g(q)=f\left(q^{\prime}\right)$, where for $\alpha<\delta, q^{\prime}(\alpha)=\left\ulcorner q_{\alpha}\right\urcorner^{\mu}$ and for $\beta \in k, q_{\alpha}(\beta)=q(\beta)(\alpha)$. If $W$ is homogeneous for $g$, then $\boldsymbol{\Pi}_{\mu}^{W}$ is homogeneous for $f$.

Proposition 10.2. Suppose $(k) \rightarrow(k)_{\omega_{1}}^{\omega_{1}}$ and $k^{+}$is a countable sequence composed of members of $k$ and ultraproducts of the form $k_{\mu}^{\prime}, k^{\prime} \subseteq k$, then

$$
\left(k^{+}\right) \rightarrow\left(k^{+}\right)^{<\omega_{1}} .
$$


Proof. Suppose $f:\left[k^{+}\right]^{\delta} \rightarrow 2, \delta<\omega_{1}$. Define $g_{\delta}:[k]^{\delta} \rightarrow 2$ by $g_{\delta}(W)=f\left(W^{(\delta)}\right)$, where

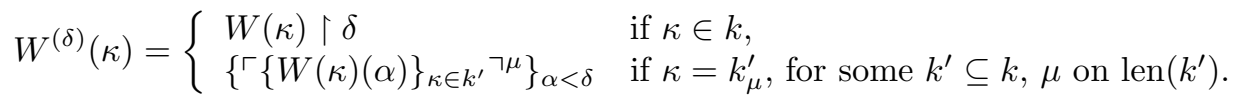

Let $X \in[k]^{k}$ be homogeneous for $g_{\delta}$. Then $X^{\prime} \in\left[k^{+}\right]^{k^{+}}$is homogeneous for $f$, where

$$
X^{\prime}(\kappa)= \begin{cases}X(\kappa) & \text { if } \kappa \in k, \\ \Pi_{\mu}^{X\left\lceil k^{\prime}\right.} & \text { if } \kappa=k_{\mu}^{\prime}, \text { for some } k^{\prime} \subseteq k, \mu \text { on } \operatorname{len}\left(k^{\prime}\right),\end{cases}
$$

since if $Y \in\left[X^{\prime}\right]^{\delta}$, as before we can construct $W \in[X]^{k}$ such that $W^{(\delta)}=Y$. The countability of $k^{+}$is needed here, along with the countable additivity of the measures.

As in the proof of Proposition 9.2, we can diagonalize the $g_{\delta}$ to obtain the result.

As in the previous section, we can prove:

Proposition 10.3. Suppose that $k, k^{+}$are as in Proposition 10.2 and there is no non-principal ultrafilter on $\omega$. Then for all cardinals $\delta$ of $\mathbf{V}, \operatorname{cof}^{\mathbf{V}^{\mathcal{P}} \omega_{1}, k^{+}}(\delta)=$ $\operatorname{cof}^{\mathbf{V}}(\delta)$ if $\operatorname{cof}^{\mathbf{V}}(\delta) \notin k^{+}$and $\operatorname{cof}^{\mathbf{V}^{\mathcal{P}} \omega_{1}, k+}(\delta)=\omega_{1}$ otherwise.

Proposition 10.4. If $k$ satisfies $(k) \rightarrow(k)_{\omega_{1}}^{k}$ and $\mu$ is a normal measure on len $(k)$, then forcing with $\mathcal{P}_{\omega_{1}, k_{\mu}}$ preserves all cardinals.

Proof. By Proposition 10.1 and Fact 1.5, we have $k_{\mu} \rightarrow\left(k_{\mu}\right)^{<\omega_{1}}$. With this, no bounded subsets of $k_{\mu}$ are added and hence all cardinals $\leq k_{\mu}$ are preserved.

Now suppose $k_{\mu} \leq \delta<\zeta$ and $p \Vdash " \dot{f}: \delta \rightarrow \zeta$, onto", $p \in \mathcal{P}_{\omega_{1}, k_{\mu}}$. Without loss of generality, we may assume $p_{1}$ to be of the form $\Pi_{\mu}^{x}$ for some $x \in[k]^{k}$, as in the proof of Proposition 9.8. Define $n_{s}$ and $n_{t}$, for $s \in\left[k_{\mu}\right]^{<\omega_{1}}$ and $t \in\left[k_{\mu}\right]^{k_{\mu}}$ and $h$ on $\left[k_{\mu}\right]^{k_{\mu}}$ as in the proof of Proposition 3.5 and Proposition 9.8. As before, $\left\|n_{t}\right\|<\zeta$. In like manner, obtain $q \in[x]^{k}$ such that whenever $r \in[q]^{k}$ and $r^{\prime} \in[r]^{k}$, then $h\left(\boldsymbol{\Pi}_{\mu}^{r}\right) \leq h\left(\boldsymbol{\Pi}_{\mu}^{r^{\prime}}\right)$.

Let $\beta=h\left(\boldsymbol{\Pi}_{\mu}^{q}\right)$. Choose $s \in\left[\boldsymbol{\Pi}_{\mu}^{q}\right]^{\xi}$ for some $\xi<\omega_{1}$ such that $\beta \in n_{s}$. Using $\mathrm{DC}$, or rather, countable choice, $s$ can be represented as $\left[s^{\prime}\right]^{\xi} / \mu$ for some $s^{\prime} \in[q]^{\xi}$. Take $y \in \prod_{\kappa \in k} \kappa$ such that $\ulcorner y\urcorner^{\mu}>\bigcup s$ and set $r=\prod_{\kappa \in k}(q(\kappa) \backslash y(\kappa))$. Let $t=\prod_{\kappa \in k}\left(r(\kappa) \cup s^{\prime}(\kappa)\right)$. Then as in Proposition 3.5,

$$
h\left(\boldsymbol{\Pi}_{\mu}^{q}\right) \leq h\left(\boldsymbol{\Pi}_{\mu}^{t}\right) \leq h\left(\boldsymbol{\Pi}_{\mu}^{r}\right)
$$

by our construction of $q$, and $h\left(\boldsymbol{\Pi}_{\mu}^{r}\right) \leq h\left(\boldsymbol{\Pi}_{\mu}^{q}\right)$ by the definition of $h$. But $\beta=$ $h\left(\boldsymbol{\Pi}_{\mu}^{r}\right)<h\left(\boldsymbol{\Pi}_{\mu}^{t}\right)$, a contradiction.

Since the value $\ulcorner p\urcorner \mu$, of a sequence $p$ in an ultrapower is independent of initial segments, we can manage both ultraproducts and ultrapowers at once. The following proposition sums this up:

Proposition 10.5. Suppose that $\left(\omega_{1} \frown k\right) \rightarrow\left(\omega_{1} \frown k\right)^{\omega_{1} \frown k}, k(0)>\omega_{1}, \omega_{1}^{\omega_{1}} / \mu=\omega_{2}$ for some normal measure $\mu$ on $\omega_{1}, k^{+}$is a countable sequence composed of members 
of $k$, ultrapowers of members of $k$, and ultraproducts of members of $k$, and that there is no non-principal ultrafilter on $\omega$. Then $\left(k^{+}\right) \rightarrow\left(k^{+}\right)^{<\omega_{1}}$. Further, $\mathcal{P}_{\omega_{1}, \omega_{2}}-k^{+}$ changes the cofinality of cardinals with cofinalities in $\omega_{2} \frown k^{+}$to $\omega_{1}$ while leaving all other cofinalities unchanged.

\section{Applications}

Theorem 11.1. $\operatorname{Con}(\mathrm{ZF}+\mathrm{AD}) \Rightarrow \operatorname{Con}\left(\mathrm{ZF}+\mathrm{DC}+\omega_{1}\right.$ is the only regular uncountable cardinal $\left.\leq \omega_{\omega_{1}+1}\right)$.

Proof. Let $\mathbf{V}^{\prime}=\mathrm{ZF}+\mathrm{AD}$, and let $\mathbf{V}=L(\mathbb{R})^{\mathbf{V}^{\prime}}$. By the work of [J1] and [J2], the remarks of section 2 and the second paragraph immediately following Definition 5.1, and Corollary 5.11, the odd $\boldsymbol{\delta}_{\alpha}^{1}$ for $\alpha<\omega_{1}$ form a sequence satisfying the strong polarized partition property such that every regular cardinal $\delta<\omega_{\omega_{1}}$ is either so that $\delta=\delta_{\alpha}^{1}$ or $\delta=\delta_{\alpha}^{1} \delta_{\alpha}^{1} / \mu$ for some normal measure $\mu$ on $\delta_{\alpha}^{1}$. By the claim on page 152 of [St2] (using $h(\alpha)=\boldsymbol{\delta}_{\alpha}^{1}$ for $\alpha<\omega_{1}$ as the function of this claim), $\omega_{\omega_{1}+1}$ is the ultraproduct as in section 10 via the unique normal measure $\mu_{\omega}$ on $\omega_{1}$ (generated by the filter of c.u.b. sets) of the odd $\boldsymbol{\delta}_{\alpha}^{1}$ for $\alpha<\omega_{1}$. Thus, since Martin has shown that assuming $\mathrm{AD}$, for $\boldsymbol{\delta}_{1}^{1}=\omega_{1}, \omega_{1} \omega_{1} / \mu_{\omega}=\omega_{2}$ (see [K12] for a proof of this fact), by Proposition 10.5, for any countable subsequence $s$ of the regular cardinals in the interval $\left[\omega_{2}, \omega_{\omega_{1}+1}\right],(s) \rightarrow(s)^{<\omega_{1}}$. This means the model $\mathbf{N}$ of Theorem 8.1 constructed using the set $A$ of regular cardinals in the interval $\left[\omega_{2}, \omega_{\omega_{1}+1}\right]$ is so that $\mathbf{N}=$ "ZF $+\mathrm{DC}+\omega_{1}$ is the only regular uncountable cardinal $\leq \omega_{\omega_{1}+1}$ ". This proves Theorem 11.1.

We remark that by Theorem 8.1 and Proposition 10.5, in $\mathbf{N}$, for $\delta$ a cardinal in $\mathbf{V}, \operatorname{cof}(\delta)=\omega_{1}$ if $\operatorname{cof}(\delta) \in A$, and $\operatorname{cof}(\delta)=\operatorname{cof}^{\mathbf{V}}(\delta)$ otherwise. Also, by Theorem 8.1 and Proposition 6.2, $\omega_{1} \omega_{1} / \mu_{\omega}=\omega_{2}$ is true in $\mathbf{N}$.

In conclusion, we remark that the desired ultimate goal is to assume $\mathbf{V}^{\prime} \models A D$, force over $\mathbf{V}=L(\mathbb{R})^{\mathbf{V}^{\prime}}$, and obtain a model $\mathbf{N}$ so that $\Theta^{\mathbf{N}}=\Theta^{\mathbf{V}}$ and $\mathbf{N}=$ "ZF $+\mathrm{DC}+\omega_{1}$ is the only regular uncountable cardinal $<\Theta$ ". (A model $\mathbf{N}$ for "ZF $+\omega_{1}$ is the only regular uncountable cardinal $<\Theta$ " in which $\mathrm{AC}_{\omega}$ is false was constructed in [A].) Theorem 8.1 provides us with a possible way of constructing such a model. If one could show that the set $A$ of Theorem 8.1 were so that $A$ could be composed of all $\mathbf{V}=L(\mathbb{R})^{\mathbf{V}^{\prime}}$-regular cardinals in the interval $\left[\omega_{2}, \Theta\right)$, then the model $\mathbf{N}$ of Theorem 8.1 would be our desired model. In fact, even though the unpublished analysis mentioned in section 2 may be difficult to extend beyond the first inaccessible, and even if the conjecture given in the last paragraph of section 2 is false, it is still conceivable that the aforementioned structural conjecture about the regular cardinals in the interval $\left[\omega_{2}, \Theta\right)$ assuming $\mathrm{AD}+\mathbf{V}=L(\mathbb{R})$ is true. Whether this is indeed the case remains open.

\section{REFERENCES}

[A] A. Apter, "AD and patterns of singular cardinals below $\Theta$," Journal of Symbolic Logic 61 (1996) 225-235. MR 97d:03071

[ER] P. Erdős and R. Rado, "A partition calculus in set theory," Bull. Amer. Math. Soc. 62 (1956) 427-489. MR 18:458a

[G] M. Gitik, "Regular cardinals in models of ZF," Trans. Amer. Math. Soc. 290 (1985) 41-68. MR 86i:03065 
[H1] J. Henle, " $\gamma$-Ramsey and $\gamma$-ineffable cardinals," Israel Journal of Mathematics 30 (1978) 85-98. MR 80a:04004

[H2] J. Henle, "Magidor-like and Radin-like forcing," Annals of Pure and Applied Logic 25 (1983) 59-72. MR 85e:03119

[H3] J. Henle, "Researches into the world of $\kappa \rightarrow(\kappa)^{\kappa}$," Annals of Mathematical Logic 17 (1979) 151-169. MR 81c:03047

[H4] J. Henle, "Some consequences of an infinite-exponent partition relation," Journal of Symbolic Logic 42 (1977) 523-526. MR 58:10461

[J1] S. Jackson, "A computation of $\boldsymbol{\delta}_{5}^{1}$," Mem. Amer. Math. Soc., to appear. CMP 98:08

[J2] S. Jackson, "AD and the projective ordinals," Cabal Seminar 81-85, Lecture Notes in Mathematics, 1333, 117-220, Springer-Verlag, 1988. MR 89k:03055

[J3] S. Jackson, "AD and the very fine structure of $L(\mathbb{R})$," Bull. Amer. Math. Soc. 21 (1989) 77-81. MR 90a:03075

[J4] S. Jackson, "Structural consequences of AD," to appear in the Handbook of Set Theory, M. Foreman, A. Kanamori, M. Magidor eds.

[J5] S. Jackson, "The weak square property," to appear in the Journal of Symbolic Logic.

[JKh] S. Jackson and F. Khafizov, "Descriptions and cardinals below $\boldsymbol{\delta}_{5}^{1}$," preprint.

[JMa] S. Jackson and D. A. Martin, "Pointclasses and well-ordered unions," Cabal Seminar 79-81, Lecture Notes in Mathematics, 1019, 56-66, Springer-Verlag, 1983. CMP 16:07

[Ke1] A. Kechris, "AD and projective ordinals," Cabal Seminar 76-77, Lecture Notes in Mathematics, 689, 91-132, Springer-Verlag, 1978. MR 80j:03069

[Ke2] A. Kechris, "The axiom of determinacy implies dependent choices in $L(\mathbb{R})$," Journal of Symbolic Logic 49 (1984) 255-267. MR 86f:03092

[KeKlMW] A. Kechris, E. Kleinberg, Y. Moschovakis, and W. H. Woodin, "The axiom of determinacy, strong partition properties, and nonsingular measures," Cabal Seminar 77-79, Lecture Notes in Mathematics, 839, 75-100, Springer-Verlag, 1981. MR 83f:03047

[KeSoSt] A. Kechris, R. Solovay, and J. Steel, "The axiom of determinacy and the pre-wellordering property," Cabal Seminar 77-79, Lecture Notes in Mathematics, 839, 101-125, Springer-Verlag, 1981. MR 83f:03042

$[\mathrm{KeW}]$ A. Kechris and W. H. Woodin, "Generic codes for uncountable ordinals, partition properties, and elementary embeddings," circulated manuscript, 1980.

[Kl1] E. Kleinberg, "AD $\vdash$ the $\aleph_{n}$ are Jonsson cardinals and $\aleph_{\omega}$ is a Rowbottom cardinal," Annals of Mathematical Logic 12 (1977) 229-248. MR 57:9550

[Kl2] E. Kleinberg, Infinitary Combinatorics and the Axiom of Determinateness, Lecture Notes in Mathematics, 612, Springer-Verlag, 1977. MR 58:109

[K13] E. Kleinberg, "Strong partition properties for infinite cardinals," Journal of Symbolic Logic 35 (1970) 410-428. MR 46:8839

[Mo1] Y. N. Moschovakis, "Ordinal games and playful models," Cabal Seminar 77-79, Lecture Notes in Mathematics, 839, 169-201, Springer-Verlag, 1981. MR 84h:03115

[Mo2] Y. Moschovakis, Descriptive Set Theory, North-Holland, Amsterdam, 1980. MR 82e:03002

[St1] J. Steel, "Closure properties of pointclasses," Cabal Seminar 77-79, Lecture Notes in Mathematics, 839, 147-163, Springer-Verlag, 1981. MR 84b:03066

[St2] J. Steel, "Scales in $L(\mathbb{R})$," Cabal Seminar 79-81, Lecture Notes in Mathematics, 1019, 107-156, Springer-Verlag, 1983. CMP 16:07

[St3] J. Steel, "HOD ${ }^{L(\mathbb{R})}$ is a core model below $\Theta$," Bulletin of Symbolic Logic 1 (1995) 75-84. MR 97a:03059

[W] W. H. Woodin, "AD and the uniqueness of the supercompact measures on $\mathcal{P}_{\omega_{1}}(\lambda)$," Cabal Seminar 79-81, Lecture Notes in Mathematics, 1019, 67-71, Springer-Verlag, 1983. CMP 16:07

Department of Mathematics, Baruch College, New York, New York 10010

E-mail address: awabb@cunyvm.cuny.edu

Department of Mathematics, Smith College, Northampton, Massachusetts 010602165

E-mail address: jhenle@smith.edu

Department of Mathematics, University of North Texas, Denton, Texas 76203

E-mail address: jackson@jove.acs.unt.edu 